\title{
Metal Complexes of Weakly Coordinating Anions. Precursors of Strong Cationic Organometallic Lewis Acids ${ }^{\dagger}$
}

\author{
WOLFGANG BECK* and KARLHEINZ SÜNKEL \\ Institut für Anorganische Chemie, Universität München, 8000 München 2, FRG
}

Received February 4, 1988 (Revised Manuscript Recelved May 2, 1988)

\section{Contents}

I. Introduction

1405

II. Scope of the Review

III. Methods of Preparation

A. Abstraction of an Anionic Ligand by a Lewis Acid

1. Abstraction of Anionic Group $X$ with Triphenylcarbenium Salts

2. Abstraction of Halide by Silver(I) and Thallium(I) Salts

3. Protonation of Hydrido, Alkyl, and $\eta^{3}$-Allyl Complexes

4. Reactions Using $\mathrm{BF}_{3}, \mathrm{AlCl}_{3}, \mathrm{NO}^{+}, \mathrm{R}_{3} \mathrm{O}^{+}, 1408$ $\mathrm{CH}_{3} \mathrm{OSO}_{2} \mathrm{~F}, \mathrm{CoBr}_{2}$, and $\mathrm{Hg}^{2+}$

B. Oxidation of Neutral Monomeric or Dimeric 1409 Complexes

C. Oxidative Addition of $R X$ to Metal $d^{8}$ Systems

D. Miscellaneous

A. Crystal Structure Determinations

B. IR Spectroscopy

C. NMR Spectroscopy

1410

1411

1411

1411

A. Relative Donor Strengths of Several Weakly Coordinating Anions

B. Substitution of Weakly Coordinating Ligands 1412 by Neutral $\sigma$ - and $\pi$-Donors

C. Reaction of Tetrafluoroborato and Hexafluorophosphato Complexes with Water

1. Formation of Aqua Complexes

2. Condensation Reactions

3. Hydrolysis of the Anion

D. Reaction with Monoanionic Ligands

E. Directed Synthesis of Ligand-Bridged Complexes

1. Neutral Ligand-Bridged Bimetallic Complexes

2. Formation of Ligand-Bridged Cationic Complexes

3. Formation of Cationic Hydride- and Acyl-Bridged Complexes

F. Miscellaneous Reactions

1. Stoichiometric Reactions

2. Catalytic Reactions
VI. Addendum

VII. Acknowledgment

VIII. Abbreviations

\section{Introduction}

Organometallic compounds of weakly coordinating anions, e.g., $\mathrm{BF}_{4}^{-}, \mathrm{PF}_{6}^{-}, \mathrm{AsF}_{6}^{-}$, and $\mathrm{SbF}_{6}^{-}$, have been the object of many studies in recent years. These complexes contain a hard anion coordinated to a soft metal center in a low oxidation state with $\pi$-acceptor ligands (e.g., $\left.\mathrm{CO}, \mathrm{PR}_{3}\right) .{ }^{1}$ They are highly reactive; the anions are excellent leaving groups and can easily be replaced by other ligands under very mild conditions, so they have proven to be useful starting materials in preparative organometallic synthesis.

The first metal carbonyl compounds of this type that could be isolated were $\mathrm{MCl}\left(\mathrm{FBF}_{3}\right)(\mathrm{NH}=\mathrm{NR})(\mathrm{CO})$ $\left(\mathrm{PPh}_{3}\right)_{2}(\mathrm{M}=\mathrm{Ru}, \mathrm{Os}),{ }^{2} \mathrm{Re}(\mathrm{CO})_{5}\left(\mathrm{FAsF}_{5}\right),{ }^{3}$ and $\mathrm{Cr}$ $(\mathrm{CO})_{3}\left(\mathrm{PMe}_{3}\right)(\Longrightarrow \mathrm{CMe})\left(\mathrm{FBF}_{3}\right){ }^{4}$ Our group in Munich entered this field when Klaus Schloter in his Master's thesis examined the reaction between $\mathrm{Mo}(\mathrm{CO})_{3}(\mathrm{Cp})$ $\left(\mathrm{SiMe}_{3}\right)$ and $\mathrm{Ph}_{3} \mathrm{C}^{+} \mathrm{BF}_{4}^{-}$. Instead of the desired $\mathrm{Me}_{2} \mathrm{Si}=\mathrm{CH}_{2}$ ligand, he discovered $\mathrm{Mo}(\mathrm{CO})_{3}(\mathrm{Cp})\left(\mathrm{FBF}_{3}\right)^{5}$ as a byproduct. Other good examples of such complexes are $\operatorname{Re}(\mathrm{CO})_{5}\left(\mathrm{FBF}_{3}\right)$ and $\mathrm{Au}\left(\mathrm{PPh}_{3}\right) \mathrm{X}$. They react as coordinatively and electronically unsaturated $\operatorname{Re}(\mathrm{CO})_{5}{ }^{+}$ or $\mathrm{Au}\left(\mathrm{PPh}_{3}\right)^{+}$with a series of nucleophiles analogously to the isolobal carbenium ions or to the proton. ${ }^{6} \mathrm{We}$ call these compounds "organometallic Lewis acids", in contrast to the "organometallic bases".? It was recognized early that all the anions mentioned above have coordinating abilities, at least in the solid state; ${ }^{8}$ however, examples are known in which in spite of electronic unsaturation of the metal, the anion remains uncoordinated $\left[\right.$ e.g., $\left[\mathrm{Rh}\left(\mathrm{PPh}_{3}\right)_{3}\right]^{+9,80}$ or $\left[\mathrm{Co}\left(\mathrm{P}(\mathrm{OR})_{3}\right)_{4}\right]^{+} \mathrm{PF}_{6}{ }^{-10}$ also in $\mathrm{Cu}(\mathrm{CO})\left(\mathrm{AsF}_{6}\right)$ the anion is apparently not coordinated to the metal, in contrast to the corresponding sulfonato complexes ${ }^{11}$ ].

The cationic fragment in these complexes must have low-lying acceptor orbitals as was shown for the $d^{6}$ $\mathrm{M}(\mathrm{CO})_{5}$ fragment. ${ }^{12 \mathrm{a}}$ A theoretical investigation of the $\left[\mathrm{Cp}_{2} \mathrm{ZrCl}\right]^{+}$moiety showed two low-lying $\delta$ - and $\pi$-acceptor MOs. ${ }^{12 \mathrm{~b}}$ It appears that the more acidic the corresponding hydrides $\mathrm{L}_{n} \mathrm{MH}$ are, the lower are the LUMOs and the higher is the electrophilicity of $\mathrm{ML}_{n}{ }^{+}$. There are many such complexes of metals in "normal" oxidation states which can only be mentioned briefly here. Examples include $\mathrm{M}(N \text {-vinylimidazole })_{4} \mathrm{SiF}_{6}(\mathrm{M}$ $=\mathrm{Mn}, \mathrm{Fe}, \mathrm{Co}, \mathrm{Ni}, \mathrm{Cu}, \mathrm{Zn}),\left[\mathrm{Ni}(\mathrm{en})_{2}\left(\mathrm{H}_{2} \mathrm{O}\right) \mathrm{BF}_{4}\right] \mathrm{BF}_{4}$, $\mathrm{Cu}\left(\mathrm{PPh}_{3}\right)_{3} \mathrm{BF}_{4}$, and $\mathrm{Mn}(2,6$-lutidine $N$-oxide $)(\mathrm{F})\left(\mathrm{BF}_{4}\right){ }^{13}$ Often the anions $\mathrm{BF}_{4}{ }^{-}$and $\mathrm{ClO}_{4}{ }^{-}$occur as bridging or "semicoordinating" ligands. ${ }^{14}$ 


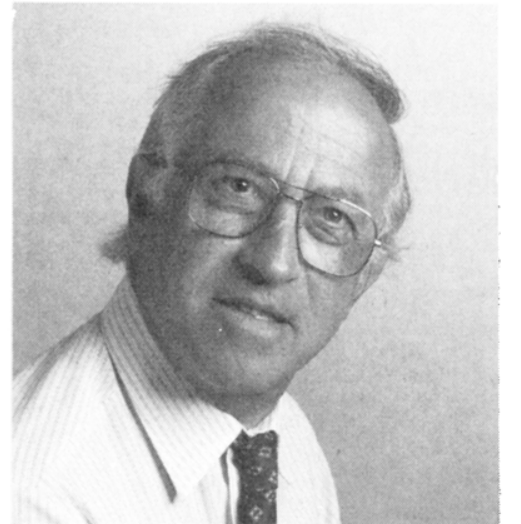

Prof. Wolfgang Beck was born in 1932 in München, West Germany. $\mathrm{He}$ received his $\mathrm{Ph} . \mathrm{D}$. under the supervision of Professor Walter Hieber at the Technische Hochschule München in 1960. He was appointed Professor of Inorganic Chemistry at the Ludwigs-Maximilians Universität München and Director of the Institute of Inorganic Chemistry in 1968. In 1977 he spent a term as Visiting Professor at the University of Wisconsin, Madison. Prof. Beck is the author of over 300 publications. He started his research with pseudohalide complexes, particularly fulminate and azido complexes, and with reactions at coordinated ligands. His current interests involve metal complexes of biologically important ligands, especially palladium and platinum compounds of $\alpha$-amino acids and their derivatives and organometallic chemistry. In the latter field he presently studies with his co-workers metal complexes of weakly coordinating anions and nucleophilic attack of organometallic anions on coordinated unsaturated hydrocarbons.

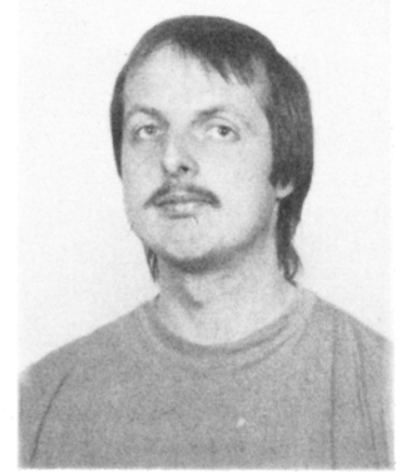

Dr. Karlheinz Sünkel was born in 1955 in München, West Germany. $\mathrm{He}$ received his Diplomchemiker degree in 1979 and his Dr. rer. nat. degree with a thesis on organometallic compounds of weakly coordinating anions under the supervision of Prof. W. Beck in 1982 at the University of Munich. After postdoctoral study with Prof. R. Bau at the University of Southern California, Los Angeles, he returned to Munich and is presently working for his "Habilitation" on complexes of functionalized cyclopentadienyls and of other unsaturated hydrocarbons.

Reviews on perchlorato, ${ }^{15}$ trifluoroacetato, ${ }^{16}$ trifluoromethanesulfonato, and fluorosulfonato ${ }^{17} \mathrm{com}$ plexes and of palladium(II) and platinum(II) compounds with such anions ${ }^{1}$ have been published. A review on the synthesis of such complexes by ion exchange has also appeared. ${ }^{18}$ The preparation of several of the compounds described here will appear in Vol. 26 of Inorganic Synthesis.

\section{Scope of the Review}

Here, we try to focus on complexes with $\pi$-acidic ligands and with the very weakly coordinating anions. $\mathrm{ClO}_{4}{ }^{-}$and $\mathrm{CF}_{3} \mathrm{SO}_{3}{ }^{-}$usually are stronger ligands and will be considered here only for the sake of comparison, where appropriate. Pentaammine fluorosulfato and triflato complexes of $\mathrm{Os}^{\mathrm{III}}, \mathrm{Co}^{\mathrm{III}}$, and $\mathrm{Ru}^{\mathrm{III}}\left[\mathrm{M}\left(\mathrm{NH}_{3}\right)_{5^{-}}\right.$ $\left.\left(\mathrm{OSO}_{2} \mathrm{R}\right)\right]^{2+}$, which have been used extensively as starting materials for many interesting complexes, e.g., $\eta^{2}$-benzene or $\eta^{2}$-acetone, ${ }^{19}$ will not be discussed here. Reactions of cationic with anionic metal complexes to give metal-metal-bonded compounds will also not be covered.

\section{Methods of Preparation}

Several methods of preparation of organometallic Lewis acids or their precursors have been developed.

\section{A. Abstraction of an Anionic Ligand by a Lewis Acid}

$$
\mathrm{L}_{n} \mathrm{M}-\mathrm{Y}+\text { Lewis acid } \rightarrow \mathrm{L}_{n} \mathrm{M}-\mathrm{X} \underset{\left[\mathrm{L}_{n} \mathrm{M}(\text { solv })\right]^{+} \mathrm{X}^{-}}{\stackrel{\text { solv }}{\longrightarrow}}
$$

\begin{tabular}{llll}
\hline $\mathrm{Y}$ & \multicolumn{1}{c}{ Lewis acid } & \multicolumn{1}{c}{$\mathrm{X}^{-}$} & solvent \\
\hline $\mathrm{H}^{-}$ & $\mathrm{H}^{+}, \mathrm{Ag}^{+}$ & $\mathrm{FBF}_{3}^{-}$ & $\mathrm{THF}$ \\
$\mathrm{CH}_{3}{ }^{-}$ & $\mathrm{Tl}^{+}, \mathrm{NO}^{+}$ & $\mathrm{FPF}_{5}{ }^{-}$ & $\mathrm{Me}_{2} \mathrm{CO}$ \\
$\mathrm{Hal}^{-}$ & $\mathrm{R}_{3} \mathrm{O}^{+}, \mathrm{Ph}_{3} \mathrm{C}^{+}$ & $\mathrm{FAsF}_{5}{ }^{-}$ & $\mathrm{H}_{2} \mathrm{O}$ \\
$\mathrm{N}_{3}^{-}$ & $\mathrm{BF}_{3}, \mathrm{AlCl}_{3}$ & $\mathrm{FSbF}_{5}{ }^{-}$ & $\mathrm{CH}_{2} \mathrm{Cl}_{2}$ \\
& $\mathrm{CH}_{3} \mathrm{OSO}_{2} \mathrm{~F}$ & $\mathrm{OClO}_{3}{ }^{-}$ & $\mathrm{CH}_{3} \mathrm{CN}$ \\
& $\mathrm{C}_{7} \mathrm{H}_{7}^{+}$ & $\mathrm{OReO}_{3}^{-}$ & $\mathrm{SO}_{2}$ \\
& $\mathrm{ArN}_{2}{ }^{+}$ & $\mathrm{OSO}_{2} \mathrm{R}^{-}$ & \\
& $\mathrm{SbCl}_{5}$ & $\mathrm{OTeF}_{5}{ }^{-}$ & \\
& $\mathrm{CoCl}_{2}$ & $\left.\mathrm{CH}_{5} \mathrm{SO}_{2} \mathrm{R}\right)_{2}{ }^{-}$ & \\
& $\mathrm{HgCl}_{2}$ & $\mathrm{ClAlCl}_{3}{ }^{-}$ &
\end{tabular}

In the complexes $\mathrm{L}_{m} \mathrm{M}-\mathrm{X}-\mathrm{EX}_{n}$ the organometallic Lewis acid competes with the Lewis acid $\mathrm{EX}_{n}$ for the base $\mathrm{X}^{-}$. In $\mathrm{AlCl}_{4}^{-}$complexes, the transition metal is usually the "winner"; i.e., formation of $\mathrm{AlCl}_{3}$ and $\mathrm{L}_{n} \mathrm{M}$ $\mathrm{Cl}$ is often observed. In the presence of coordinating solvents (e.g., THF, $\mathrm{CH}_{3} \mathrm{CN}$, liquid $\mathrm{SO}_{2}$ ) the cationic solvent complexes $\left[\mathrm{L}_{n} \mathrm{M}(\mathrm{solv})\right]^{+}$are formed. From some cationic $\mathrm{SO}_{2}$ complexes the neutral $\mathrm{L}_{n} \mathrm{MX}$ can be obtained by simple evacuation. ${ }^{3,20}$

\section{Abstraction of Anionic Group $X$ with Triphenylcarbenium Salts}

The reaction of iridium hydrido complexes with trityl salts to give triphenylmethane was first reported in $1962 .{ }^{21}$ The organometallic product was not studied. A ruthenium dihydride yielded a cationic monohydrido complex with no coordination of anion or solvent. ${ }^{22}$

$$
\begin{array}{r}
\mathrm{RuH}_{2}\left(\mathrm{PPh}_{3}\right)_{4}+\mathrm{Ph}_{3} \mathrm{C}^{+} \mathrm{BF}_{4}^{-} \rightarrow \\
{\left[\mathrm{RuH}\left(\mathrm{PPh}_{3}\right)_{4}\right]^{+} \mathrm{BF}_{4}^{-}+\mathrm{Ph}_{3} \mathrm{CH}}
\end{array}
$$

However, phosphine transfer was observed in the analogous reaction of the corresponding iron hydride. ${ }^{22 a}$

A cationic complex $\left[\mathrm{CpW}(\mathrm{NO})_{2} \mathrm{NCMe}^{+}\right.$with coordinated solvent was obtained in the reaction of $\mathrm{CpW}$ (NO) ${ }_{2} \mathrm{H}$ with $\mathrm{Ph}_{3} \mathrm{C}^{+} \mathrm{BF}_{4}^{-}$in $\mathrm{CH}_{3} \mathrm{CN} .^{23}$ The first time a complex of coordinated $\mathrm{BF}_{4}^{-}$could be obtained by this route was the synthesis of $\mathrm{Cp}(\mathrm{CO})_{3} \mathrm{MoFBF}_{3}$ from $\mathrm{CpMo}(\mathrm{CO})_{3} \mathrm{H}$ and $\mathrm{Ph}_{3} \mathrm{CBF}_{4}$ in $\mathrm{CH}_{2} \mathrm{Cl}_{2}{ }^{5}$ A similar reaction of $\mathrm{Mn}(\mathrm{CO})_{4}\left(\mathrm{PR}_{3}\right) \mathrm{H}$ in moist $\mathrm{CH}_{2} \mathrm{Cl}_{2}$ gave the aqua complex $\left[\mathrm{Mn}(\mathrm{CO})_{4}\left(\mathrm{PR}_{3}\right)\left(\mathrm{H}_{2} \mathrm{O}\right)\right]^{+} \mathrm{BF}_{4}^{--24}(\mathrm{OC})_{5} \mathrm{ReH}$ and $\mathrm{Ph}_{3} \mathrm{C}^{+} \mathrm{BF}_{4}^{-}$afford (OC) ${ }_{5} \mathrm{ReFBF}_{3}{ }^{42}$ Molybdenum and tungsten compounds of coordinated $\mathrm{PF}_{6}{ }^{-}, \mathrm{AsF}_{6}{ }^{-}$, 
or $\mathrm{SbF}_{6}{ }^{-}$could be obtained in the same manner: ${ }^{25}$

$\left.\mathrm{CpMo}(\mathrm{CO})_{3} \mathrm{H}+\mathrm{Ph}_{3} \mathrm{C}^{+} \mathrm{X}^{-} \stackrel{\mathrm{CH}_{2} \mathrm{Cl}_{2}}{\longrightarrow} \mathrm{CpMo} \mathrm{CO}\right)_{3} \mathrm{X}$
$\left(\mathrm{X}=\mathrm{FBF}_{3}, \mathrm{FPF}_{5}, \mathrm{FAsF}_{5}, \mathrm{FSbF}_{5}\right)+\mathrm{Ph}_{3} \mathrm{CH}$

Recently, the synthesis of the analogous pentamethylcyclopentadienyl complexes $\left(\mathrm{C}_{5} \mathrm{Me}_{5}\right) \mathrm{Mo}(\mathrm{CO})_{3^{-}}$ $\left(\mathrm{BF}_{4}, \mathrm{PF}_{6}\right)$ by this method and its reactions have been reported.26a 'It may be noted in this context that the hydride $\mathrm{CpW}(\mathrm{CO})_{3} \mathrm{H}$ reacts with $\mathrm{Ph}_{3} \mathrm{C}$ radical in the presence of $\mathrm{Ph}_{3} \mathrm{CCl}$ and $\mathrm{PR}_{3}$ to form $\mathrm{CpW}(\mathrm{CO})_{3} \mathrm{Cl}$ and $\mathrm{CpW}(\mathrm{CO})_{2}\left(\mathrm{PR}_{3}\right) \mathrm{Cl}^{26 \mathrm{~b}}$

Fenske-Hall calculations show the more acidic character of the hydrogen atom in $\mathrm{CpMo}(\mathrm{CO})_{3} \mathrm{H}$ compared to $\mathrm{CpMo}(\mathrm{NO}){ }_{2} \mathrm{H},{ }^{27}$ but as these reactions show, the hydrogen atom can be abstracted as hydride in both cases. The dinuclear dihydride $[\mathrm{CpFe}(\mathrm{CO}) \mathrm{H}]_{2}(\mu$-dppe $)$ gave the hydride-bridged $\left[[\mathrm{CpFe}(\mathrm{CO})]_{2}(\mu-\mathrm{H})(\mu-\right.$ dppe) $]^{+} \mathrm{BF}_{4}{ }^{28}$ Interestingly, in one case an intermediate $\eta^{2}$-triphenylmethane complex could be isolated ${ }^{29 a}$

$$
\begin{gathered}
\mathrm{Cp}(\mathrm{ON})(\mathrm{OC}) \mathrm{Re}-\mathrm{H}+\mathrm{Ph}_{3} \mathrm{C}^{+} \mathrm{PF}_{6}^{-} \rightarrow \\
{\left[\mathrm{Cp}(\mathrm{ON})(\mathrm{OC}) \operatorname{Re}\left(\eta^{2}-\mathrm{C}_{6} \mathrm{H}_{5} \mathrm{CHPh}_{2}\right)\right]^{+} \mathrm{PF}_{6}^{-}}
\end{gathered}
$$

in which the $\eta^{2}$-arene ligand is readily displaced by $\mathrm{PPh}_{3}$ and can be deprotonated to give $\operatorname{Cp}(\mathrm{ON})(\mathrm{OC}) \operatorname{Re}\left(\eta^{1}\right.$ $\mathrm{C}_{6} \mathrm{H}_{4} \mathrm{CHPh}_{2}$ ). The abstraction of hydride from hydrido complexes in the presence of ligands has been used for the preparation of various cationic complexes $\left(\mathrm{L}=\mathrm{H}_{2} \mathrm{O}\right.$, THF, NCMe): $\left[\mathrm{CpOs}(\mathrm{CO})_{2}(\mathrm{~L})\right]^{+},{ }^{30}[\mathrm{CpRe}(\mathrm{NO})(\mathrm{CO})-$ $\mathrm{L}^{+}, 31\left[\mathrm{Mn}(\mathrm{CO})_{4}\left(\mathrm{PR}_{3}\right) \mathrm{L}\right]^{+},{ }^{24}\left[\mathrm{Mn}(\mathrm{CO})_{3}\left(\mathrm{P}(\mathrm{OR})_{3}\right)_{2} \mathrm{~L}\right]^{+}, 32$ $\left[\mathrm{CpW}(\mathrm{NO})_{2} \mathrm{~L}\right]^{+},{ }^{23}$ and $\left[\mathrm{Cp}_{2} \mathrm{Nb}(\mathrm{CO})(\mathrm{L})\right]^{+}{ }^{33}$ The $[\mathrm{Co}-$ $\left.(\mathrm{CO})_{3} \mathrm{~L}\right]^{+}$system ( $\mathrm{L}=\mathrm{CO}, \mathrm{PPh}_{3}$ ) is obviously too unstable to be isolated, since the reaction of $\mathrm{HCo}(\mathrm{CO})_{3} \mathrm{~L}$ with $\mathrm{Ph}_{3} \mathrm{C}^{+}$gave only $\left[\mathrm{Co}(\mathrm{CO})_{3} \mathrm{~L}\right]_{2}$ and cobalt(II)..$^{34,35}$ However, $\mathrm{HCo}\left(\mathrm{P}(\mathrm{OPh})_{3}\right)_{4}$ is oxidized to $[\mathrm{HCo}(\mathrm{P}$ $\left.\left.(\mathrm{OPh})_{3}\right)_{4}\right]^{+}$by $\mathrm{Ph}_{3} \mathrm{C}^{+36}$ (for the reaction with acid see below). The abstraction of hydride from the anionic complex $\left[\mathrm{CpV}(\mathrm{CO})_{3} \mathrm{H}\right]^{-}$led to $\mathrm{CpV}(\mathrm{CO})_{3} \mathrm{~L}{ }^{37}$ The polyhydride $\mathrm{Os}\left(\mathrm{PR}_{3}\right)_{3} \mathrm{H}_{4}$ gave $\left[\mathrm{Os}\left(\mathrm{PR}_{3}\right)_{3}\left(\mathrm{CH}_{3} \mathrm{CN}\right)_{3}\right]^{2+} .38$ Formation of $\mathrm{Ph}_{3} \mathrm{CH}$ from metal hydrides and trityl salts is further evidence for the negative charge ${ }^{39,40}$ on the hydrogen atom in these metal hydrides.

Abstraction of a methyl group by trityl cation has been reported first for $\mathrm{CpFe}(\mathrm{CO})_{2} \mathrm{Me}$ to yield the aqua complex $\left[\mathrm{CpFe}(\mathrm{CO})_{2}\left(\mathrm{H}_{2} \mathrm{O}\right)\right]^{+} .41$ Also $\mathrm{Mn}(\mathrm{CO})_{4}\left(\mathrm{PR}_{3}\right)$ $\mathrm{Me}^{24}$ and $\mathrm{M}(\mathrm{CO})_{5} \mathrm{Me}(\mathrm{M}=\mathrm{Mn}, \mathrm{Re})^{42}$ gave with $\mathrm{Ph}_{3} \mathrm{C}^{+}$ $\left[\mathrm{Mn}(\mathrm{CO})_{4}\left(\mathrm{PR}_{3}\right)\left(\mathrm{H}_{2} \mathrm{O}\right)\right]^{+}$and $\mathrm{M}(\mathrm{CO})_{5} \mathrm{FBF}_{3}$, respectively. On the other hand, $\mathrm{Ph}_{3} \mathrm{C}^{+}$abstracts hydride from the methyl group in $\mathrm{CpRe}(\mathrm{NO})\left(\mathrm{PPh}_{3}\right)\left(\mathrm{CH}_{3}\right)^{43}$ and $\mathrm{Cp}_{2} \mathrm{~W}$ $\left(\mathrm{CH}_{2} \mathrm{R}\right)_{2}{ }^{44}$ to afford the cationic methylene complexes $\left[\mathrm{CpRe}(\mathrm{NO})\left(\mathrm{PPh}_{3}\right)\left(\mathrm{CH}_{2}\right)\right]^{+}$and $\left[\mathrm{Cp}_{2} \mathrm{~W}\left(\mathrm{CH}_{2} \mathrm{R}\right)(\mathrm{CHR})\right]^{+}$, respectively. Carbene complexes of metals in higher oxidation states are also obtained by hydride abstraction from alkyl ligands. ${ }^{45}$

Evidence for initial electron transfer from the metal-alkyl compound to the trityl cation has been found in a few cases. $430-46-48$ Usually, with longer alkyl groups $\beta$-hydride elimination occurs and cationic $\eta^{2}$-alkene complexes can be isolated. ${ }^{41,43 \mathrm{c}}$ Use of acylium cations instead of trityl salts leads to the formation of $\eta^{1}$ aldehyde and ketone complexes. ${ }^{49}$

$$
\mathrm{L}_{n} \mathrm{M}-\mathrm{R}+\mathrm{PhCO}^{+} \mathrm{SbF}_{6}{ }^{-} \rightarrow\left[\mathrm{L}_{n} \mathrm{M}(\mathrm{PhCOR})\right]^{+} \mathrm{SbF}_{6}{ }^{-}
$$

$$
\mathrm{L}_{n} \mathrm{M}=\mathrm{Re}(\mathrm{CO})_{5}, \mathrm{CpW}(\mathrm{CO})_{3} ; \mathrm{R}=\mathrm{H}, \mathrm{CH}_{3}
$$

\section{Abstraction of Halide by Silver(I) and Thallium(I) Salts}

The simple metathesis of halide with the weaker coordinating anions by means of silver salts has been used in numerous cases and not all examples can be cited here. Either cationic solvent complexes or complexes with coordinating anions could be obtained, e.g., $\left[\mathrm{CpFe}(\mathrm{L}) \mathrm{L}^{\prime}(\mathrm{solv})\right]^{+},{ }^{50-52}\left[\mathrm{CpFe}(\mathrm{CO})_{2}\left(\mathrm{FBF}_{3}{ }^{53} \mathrm{OTeF}_{5}{ }^{54 a}\right)\right]$, $\left[(\mathrm{Mn}, \mathrm{Re})(\mathrm{CO})_{3} \mathrm{~L}_{2}\left(\mathrm{OClO}_{3},{ }^{55,56 \mathrm{a}, \mathrm{b}} \mathrm{FBF}_{3},{ }^{, 66 \mathrm{C}} \mathrm{OReO}_{3},{ }^{57} \mathrm{FAs}-\right.\right.$ $\left.\left.\mathrm{F}_{5},{ }^{3} \mathrm{OSO}_{2} \mathrm{CF}_{3},{ }^{58} \mathrm{OSO}_{2} \mathrm{~F}^{, 69 \mathrm{a}} \mathrm{CH}\left(\mathrm{SO}_{2} \mathrm{~F}\right)_{2}{ }^{59 \mathrm{~b}}\right)\right][\mathrm{L}=\mathrm{CO}$, $\left.\mathrm{PR}_{3}\right]$, [( $\left.\left.\mathrm{Mn}, \mathrm{Re}\right)(\mathrm{CO})_{3} \mathrm{~L}_{2}\left(\mathrm{H}_{2} \mathrm{O}\right)\right]^{+}{ }^{56 \mathrm{c}} \quad\left[\mathrm{CpCr}(\mathrm{NO})_{2^{-}}\right.$ (NCMe) $]^{+},{ }^{60}\left[\mathrm{Pd}(\mathrm{L})_{2}(\mathrm{solv})_{2}\right]^{2+}\left(\mathrm{L}=1 /{ }_{2} \mathrm{dppe},{ }^{61 \mathrm{a}} \mathrm{COD}\right.$, $\left.\mathrm{nbd}^{61 \mathrm{~b}}\right), \operatorname{Ir}(\mathrm{CO})\left(\mathrm{PPh}_{3}\right)_{2}\left(\mathrm{OClO}_{3}\right)$ and $\left[\operatorname{Ir}(\mathrm{CO})\left(\mathrm{PPh}_{3}\right)_{2^{-}}\right.$ (solv) ${ }^{+},{ }^{62} \mathrm{Pt}$ (nbd) $\left.(\mathrm{OTeF})_{2}\right)_{2}{ }^{63}\left[\mathrm{Ru}(\mathrm{dppe})_{2} \mathrm{~L}_{2}\right]^{2+}, 64[\mathrm{Ru}-$ (COD)(NCMe $\left.)_{4}\right]^{2+},{ }^{65 \mathrm{a}}\left[\mathrm{Rh}(\mathrm{COD})(\text { solv })_{x}\right]^{+66}\left[\mathrm{Cp}_{2} \mathrm{Zr}-\right.$ $(\mathrm{Me})(\mathrm{NCMe})]^{+}, 67 \mathrm{a}^{\prime} \mathrm{Cp}_{2} \mathrm{Zr}\left(\mathrm{OSO}_{2} \mathrm{CF}_{3}\right)_{2}$ (THF), ${ }^{67 \mathrm{~b}}$ and $\left[\left(\mathrm{C}_{5} \mathrm{Me}_{5}\right) \mathrm{M}(\mathrm{NCMe})_{3}\right]^{2+}(\mathrm{M}=\mathrm{Rh}, \mathrm{Ir}){ }^{68}\left[\mathrm{Mn}(\mathrm{CO})_{3^{-}}\right.$ $\left.(\mathrm{NCMe})_{3}\right]^{+}$has been shown to be a useful precursor for cationic manganese carbonyl compounds. ${ }^{65 b}$ The complex $\mathrm{CpFe}(\mathrm{CO})_{2} \mathrm{FBF}_{3}$ from $\mathrm{CpFe}(\mathrm{CO})_{2} \mathrm{I}$ and $\mathrm{AgBF}_{4}$ is formed via the iodo-bridged complex $\left[\left(\mathrm{CpFe}(\mathrm{CO})_{2}\right]_{2}(\mu\right.$ I) $]^{+} \mathrm{BF}_{4}{ }^{-53}$ The mechanism of chloride abstraction in $\mathrm{Mo}(\mathrm{NO})_{2} \mathrm{~L}_{2} \mathrm{Cl}_{2}$ has been also investigated. ${ }^{69}$ These reactions have been carried out often in the presence of other ligands without isolation of any organometallic Lewis acids.

It should be pointed out that sometimes the silver atom gets attached to the molecule. ${ }^{64,70,71}$

In a few cases $\mathrm{Tl}^{+}$salts could also be used; e.g., $\mathrm{WCl}_{2} \mathrm{H}_{2}\left(\mathrm{PMe}_{3}\right)_{4}$ and $\mathrm{TlBF}_{4}$ in $\mathrm{CH}_{3} \mathrm{CN}$ give [WCl$\left.(\mathrm{NCMe}) \mathrm{H}_{2}\left(\mathrm{PMe}_{3}\right)_{4}\right]^{+} \mathrm{BF}_{4}^{-}$, while in $\mathrm{C}_{6} \mathrm{H}_{5} \mathrm{Cl}$ the $16 \mathrm{e}$ complex $\left[\mathrm{WClH}_{2}\left(\mathrm{PMe}_{3}\right)_{4}\right]^{+}$is formed. ${ }^{72}$ Also $\left[\mathrm{AuPR}_{3}\right]^{+}$ can be generated in situ from $\mathrm{AuPR}_{3} \mathrm{Cl}$ and $\mathrm{TlBF}_{4}{ }^{73}$ $\mathrm{CpFe}(\mathrm{dmpe}) \mathrm{I}$ and $\mathrm{TlBF}_{4}$ give $[\mathrm{CpFe}(\mathrm{dmpe})(\mathrm{solv})]^{+}$. $\mathrm{BF}_{4}{ }^{74}$ while with $\mathrm{AgBF}_{4}$ the $17 \mathrm{e}$ cation $[\mathrm{CpFe}(\mathrm{dmpe}) \mathrm{I}]^{+}$ is obtained.

\section{Protonation of Hydrido, Alkyl, and $\eta^{3}-A l l y l$ Complexes}

The elimination of hydrogen by reaction of hydrido complexes with strong acids in the presence of other neutral ligands has often been studied, since the protonation of several hydrido complexes in several strong acids was examined in 1962: ${ }^{75}$

$$
\mathrm{ML}_{n} \mathrm{H}+\mathrm{HX}+\mathrm{L}^{\prime} \rightarrow\left[\mathrm{ML}_{n} \mathrm{~L}^{\prime}\right]^{+} \mathrm{X}^{-}+\mathrm{H}_{2}
$$

In several cases the first step of this reaction may be the formation of $\eta^{2}$-dihydrogen complexes, as has been proven by the isolation of $\left[\mathrm{P}\left(\mathrm{CH}_{2} \mathrm{CH}_{2} \mathrm{PPh}_{2}\right)_{3} \mathrm{RhH}_{2}\right]^{+}$, $6 \mathrm{a}$ $\left[\mathrm{FeH}_{3}(\mathrm{dppe})_{2}\right]^{+}{ }^{766, \mathrm{~d}}$ and $\left[\mathrm{CpRu}(\mathrm{CO})\left(\mathrm{PR}_{3}\right) \mathrm{H}_{2}\right]^{+} \mathrm{BF}_{4}{ }^{-76 \mathrm{c}}$ Also other protonated hydrido complexes, like $\left[\mathrm{H}_{2} \mathrm{Co}-\right.$ $\left.\left[\mathrm{P}(\mathrm{OR})_{3}\right]_{4}\right]^{+},{ }^{10}$ originally formulated as dihydrido compounds, might be $\eta^{2}$-dihydrogen complexes. ${ }^{76 e}$ Nitrosyl hydrides as $\mathrm{CpW}(\mathrm{NO})_{2} \mathrm{H}^{23}$ or $\mathrm{Mn}(\mathrm{NO})_{2}\left(\mathrm{PPh}_{3}\right)_{2} \mathrm{H}^{77}$ yield with acids $\mathrm{HX} \mathrm{CpW(NO)}{ }_{2} \mathrm{OTs}$ and $\mathrm{Mn}(\mathrm{NO})_{2}\left(\mathrm{PPh}_{3}\right)_{2} \mathrm{X}$ $\left(\mathrm{X}=\mathrm{FBF}_{3}, \mathrm{OSO}_{2} \mathrm{CF}_{3}\right)$, respectively.

Many polyhydrides, e.g., $\mathrm{MoL}_{4} \mathrm{H}_{4},{ }^{78,79} \mathrm{OsH}_{4} \mathrm{~L}_{3}{ }^{38,80}$ and $\mathrm{ReH}_{5}\left(\mathrm{PR}_{3}\right)_{2} \mathrm{~L}$, 11 have been reacted with acids. Whereas sometimes all hydrogens remain in the complex after protonation, $80,81 \mathrm{~b}, 82$ it also happens that one or more molecules of $\mathrm{H}_{2}$ are lost. ${ }^{80,83}$ A few interesting reactions have been observed with dihydrides. Thus, while $\mathrm{ReH}_{2} \mathrm{NO}\left(\mathrm{PPh}_{3}\right)_{3}$ yields with $\mathrm{HClO}_{4}$ in the presence of $\mathrm{CO}$ and alcohols the complexes $\mathrm{ReH}(\mathrm{OR})(\mathrm{CO})(\mathrm{NO})$ $\left(\mathrm{PPh}_{3}\right)_{2},{ }^{84 \mathrm{a}}$ a fluoro cation $\left[\mathrm{Re}(\mathrm{F})(\mathrm{CO})(\mathrm{NO})\left(\mathrm{PPh}_{3}\right)_{3}\right]^{+}$is 
obtained with $\mathrm{HBF}_{4} / \mathrm{CO}{ }^{84 \mathrm{~b}} \mathrm{RuH}_{2}\left(\mathrm{PPh}_{3}\right)_{4}$ yields on protonation with $p$ - $\mathrm{TsOH},{ }^{85 \mathrm{a}} \mathrm{H}_{2} \mathrm{C}\left(\mathrm{SO}_{2} \mathrm{CF}_{3}\right)_{2} /$ toluene, ${ }^{85 \mathrm{~b}}$ or $\mathrm{HBF}_{4}$ or phenol ${ }^{85 \mathrm{c}} \eta^{6}$-arene complexes [RuH$\left(\mathrm{PPh}_{3}\right)_{2}$ (arene) $]^{n+}(n=0,1)$, where the arene is either the phenyl ring of $\mathrm{OTs}^{-}$, toluene, $\mathrm{PPh}_{3}$, or $\mathrm{OPh}^{-}$. Also $\mathrm{OsH}_{4}\left(\mathrm{PPh}_{3}\right)_{3}$ undergoes a similar reaction with sulfonic acids in refluxing benzene. ${ }^{85 \mathrm{~d}}$

This method is especially convenient for metal carbonyl systems where the carbonyl metalate can be protonated without isolation of the intermediate hydride: complexes $\left[\mathrm{Fe}(\mathrm{CO})_{3}(\mathrm{NO}) \mathrm{L}\right]^{+86}$ and $\mathrm{Mn}(\mathrm{CO})_{5} \mathrm{O}$ $\mathrm{SO}_{2} \mathrm{CF}_{3}{ }^{87}$ have been produced from $\left[\mathrm{Fe}(\mathrm{CO})_{3}(\mathrm{NO})\right]^{-}$and $\left[\mathrm{Mn}(\mathrm{CO})_{5}\right]^{-}$, respectively.

The best and cleanest method for the isolation of organometallic Lewis acids seems to be the protonation of methyl complexes:

$$
\mathrm{L}_{n} \mathrm{M}-\mathrm{CH}_{3}+\mathrm{HX} \rightarrow \mathrm{L}_{n} \mathrm{M}-\mathrm{X}+\mathrm{CH}_{4}
$$

This method made the isolation of the following complexes possible: $\operatorname{Re}(\mathrm{CO})_{5} \mathrm{FBF}_{3},{ }^{88} \mathrm{CpM}(\mathrm{CO})(\mathrm{L})\left(\mathrm{OSO}_{2} \mathrm{R}\right)$ $\left(\mathrm{M}=\mathrm{Fe}, \mathrm{Ru}, \mathrm{L}=\mathrm{CO}, \mathrm{PMe}_{3}\right){ }^{89} \mathrm{Cp}(\mathrm{OC})_{2} \mathrm{FeOTeF}_{5},{ }^{54}$ $\mathrm{CpM}(\mathrm{CO})_{3} \mathrm{X}\left(\mathrm{M}=\mathrm{Mo}, \mathrm{W} ; \mathrm{X}=\mathrm{FBF}_{3}, \mathrm{OSO}_{2} \mathrm{R}\right),{ }^{90} \mathrm{M}$ (CO) ${ }_{5} \mathrm{OTeF}_{5}(\mathrm{M}=\mathrm{Mn}, \mathrm{Re}),{ }^{54} \mathrm{CpRe}(\mathrm{NO})\left(\mathrm{PPh}_{3}\right) \mathrm{X}(\mathrm{X}$ $\left.=\mathrm{OSO}_{2} \mathrm{CF}_{3}, \mathrm{FBF}_{3}, \mathrm{FPF}_{5}\right){ }^{91}$

However, it could be shown that isolation of these complexes and of " $\left(\mathrm{C}_{6} \mathrm{H}_{6}\right) \mathrm{Ru}\left(\mathrm{PR}_{3}\right)\left(\mathrm{CH}_{3}\right)^{+"}$ is not necessary for many reactions. ${ }^{92-95}$ Also ethy ${ }^{96}$ and other $\sigma$-alkyl and $\sigma$-ally ${ }^{97}$ complexes can be used. $\mathrm{H}_{3} \mathrm{CCo}$ $\left[\mathrm{P}(\mathrm{OR})_{3}\right]_{4}$ can be protonated by $\mathrm{NH}_{4} \mathrm{PF}_{6}$ to give $\left[\left(\mathrm{H}_{3} \mathrm{~N}\right) \mathrm{Co}\left[\mathrm{P}(\mathrm{OR})_{3}\right]_{4}\right]^{+} \mathrm{PF}_{6}{ }^{-10}$

Some mechanistic investigations on the reaction of metal alkyl complexes with strong acids have been undertaken. ${ }^{98,99}$ The protonation of the optically active $\mathrm{CpRe} *(\mathrm{NO})\left(\mathrm{PPh}_{3}\right) \mathrm{R}^{*}$ by $\mathrm{HBr}$ proceeds with retention of configuration at both carbon and rhenium, ${ }^{98}$ which "can be visualized as a net addition of $\mathrm{HX}$ across the rhenium-carbon $\sigma$-bond".

The intermediate complex $\left[\mathrm{Cp}\left(\mathrm{Me}_{3} \mathrm{P}\right)_{2} \mathrm{Ru}\left(\mathrm{CH}_{3}\right) \mathrm{H}\right]^{+}$. $\mathrm{BF}_{4}^{-}$from the protonation of $\mathrm{Cp}\left(\mathrm{Me}_{3} \mathrm{P}\right){ }_{2} \mathrm{Ru}\left(\mathrm{CH}_{3}\right)$ with $\mathrm{HBF}_{4}$ could be isolated. ${ }^{99 \mathrm{~b}}$ Further examples for the cleavage of the metal-carbon $\sigma$-bond by protonic acids are summarized in ref 100 .

The cleavage of the cyclopentadienyl ligand from nickelocene via $[\mathrm{NiCp}]^{+}$has led to the first "tripledecker" compound $\left[\mathrm{Ni}_{2} \mathrm{Cp}_{3}\right]^{+}{ }^{101 a}$ which can be used as an excellent source for the extremely reactive $[\mathrm{NiCp}]^{+}$ unit. ${ }^{101 \mathrm{~b}}$

Interestingly, protonation of $\left(\eta^{6}-\mathrm{C}_{7} \mathrm{H}_{8}\right) \mathrm{M}(\mathrm{CO})_{3}(\mathrm{M}=$ $\mathrm{Mo}, \mathrm{W})$ by $\mathrm{HBF}_{4}$ yields the $16 \mathrm{e}$ system $\left[\left(\mathrm{C}_{7} \mathrm{H}_{9}\right) \mathrm{M}\right.$ $\left.(\mathrm{CO})_{3}\right]^{+} \mathrm{BF}_{4}^{-}$with no coordination of the anion. ${ }^{102}$ In contrast to the analogous tungsten compound, the molybdenum atom is not electrophilic, since addition of phosphine takes place at the $\mathrm{C}_{7} \mathrm{H}_{9}$ group.

In contrast to these reactions, the cyclohexadienyl complex $\mathrm{CpFeC}_{6} \mathrm{H}_{7}$ is protonated by $\mathrm{HBF}_{4} \cdot \mathrm{OEt}_{2}$ to give the very reactive diene-tetrafluoroborato complex $\mathrm{CpFe}\left(\eta^{4}-\mathrm{C}_{6} \mathrm{H}_{8}\right)\left(\mathrm{FBF}_{3}\right)$. $^{103}$ Protonation of the $\eta^{3}$-allyl complex $\mathrm{CpMo}(\mathrm{CO})_{2}\left(\mathrm{C}_{3} \mathrm{H}_{5}\right)$ with $\mathrm{HBF}_{4}$ yielded $\mathrm{Cp}$ (CO) ${ }_{2} \mathrm{Mo}\left(\mathrm{CH}_{2}=\mathrm{CHMe}\right)\left(\mathrm{FBF}_{3}\right)$, an organometallic Lewis acid bearing two accessible coordination sites (eq 8). ${ }^{104 a}$ Similarly, protonation of $\eta^{3}$-allyl complexes $\left(\mathrm{C}_{3} \mathrm{H}_{5}\right) \mathrm{ML}_{n}\left(\mathrm{ML}_{n}=\mathrm{Co}\left[\mathrm{P}(\mathrm{OMe})_{3}\right]_{3},{ }^{10} \mathrm{Mn}(\mathrm{CO})_{2}[\mathrm{P}(\mathrm{O}-i-\right.$ $\left.\left.\mathrm{C}_{3} \mathrm{H}_{7}\right)_{3}\right]_{2},{ }^{104 \mathrm{~b}}$ and $\left.\mathrm{Ru}\left(\eta^{4} \text {-diene }\right)^{104 c}\right)$ gives compounds with two reactive coordination sites. $\mathrm{Ni}\left(\eta^{3}-\mathrm{C}_{3} \mathrm{H}_{5}\right), \mathrm{PMe}_{3}$, and $\mathrm{HBF}_{4}$ yield $\left(\eta^{3}-\mathrm{C}_{3} \mathrm{H}_{5}\right) \mathrm{Ni}\left(\mathrm{PMe}_{3}\right)\left(\mathrm{FBF}_{3}\right)$, which was characterized by ${ }^{31} \mathrm{P}$ NMR (eq 9). ${ }^{105}$

$$
\begin{gathered}
\mathrm{Cp}(\mathrm{OC})_{2} \mathrm{Mo}\left(\mathrm{CH}_{2} \mathrm{CHCH}_{2}\right)+\mathrm{HBF}_{4} \rightarrow \\
\mathrm{Cp}(\mathrm{OC})_{2} \mathrm{Mo}\left(\mathrm{CH}_{2}=\mathrm{CHCH}_{3}\right)\left(\mathrm{FBF}_{3}\right) \\
\mathrm{Ni}_{\left(\mathrm{CH}_{2} \mathrm{CHCH}_{2}\right)_{2}+\mathrm{HBF}_{4}+\mathrm{PMe}_{3} \rightarrow} \rightarrow \\
\left(\mathrm{CH}_{2} \mathrm{CHCH}_{2}\right) \mathrm{Ni}\left(\mathrm{PMe}_{3}\right)\left(\mathrm{FBF}_{3}\right)+\mathrm{CH}_{2}=\mathrm{CHCH}_{3}
\end{gathered}
$$

The aryl complexes $\mathrm{CpRe}(\mathrm{CO})(\mathrm{NO})\left(\mathrm{C}_{6} \mathrm{H}_{4} \mathrm{R}\right)$ gave cationic $\eta^{2}$-arene complexes [CpRe(CO)(NO) $\left(\eta^{2}\right.$ $\left.\left.\mathrm{C}_{6} \mathrm{H}_{5} \mathrm{R}\right)\right]^{+}$upon protonation with $\mathrm{HBF}_{4}{ }^{29 \mathrm{~b}}$ Evidence for the formation of $\mathrm{CpFe}(\mathrm{CO})_{2} \mathrm{BF}_{4}$ from $\mathrm{CpFe}$ (CO) ${ }_{2} \mathrm{SO}_{2} \mathrm{OR}$ and $\mathrm{HBF}_{4}$ has been reported. ${ }^{106}$

Closely related are the reactions of metal alkyl complexes with metal hydrides to give alkanes and dimeric species with metal-metal bonds. ${ }^{107}$

\section{Reactions Using $\mathrm{BF}_{3}, \mathrm{AlCl}_{3}, \mathrm{NO}^{+}, \mathrm{R}_{3} \mathrm{O}^{+}$, $\mathrm{CH}_{3} \mathrm{OSO}_{2} \mathrm{~F}, \mathrm{COBr}_{2}$, and $\mathrm{Hg}^{2+}$}

A general applicable method for the synthesis of cationic metal carbonyl complexes is the halide abstraction promoted by $\mathrm{BF}_{3}$ or $\mathrm{AlCl}_{3}$ in the presence of neutral ligands; ${ }^{108-111}$ e.g.

$$
\mathrm{L}_{n} \mathrm{M}-\mathrm{Cl}+\mathrm{AlCl}_{3} \rightarrow \mathrm{L}_{n} \mathrm{MClAlCl}_{3} \stackrel{+\mathrm{L}^{\prime}}{\longrightarrow}
$$

Thus the first homoleptic metal carbonyl cations [M$\left.(\mathrm{CO})_{6}\right]^{+}(\mathrm{M}=\mathrm{Mn}, \mathrm{Re})$ could be isolated. ${ }^{108,109}$ In several cases evidence for the intermediacy of coordinated $\mathrm{AlCl}_{4}{ }^{-}$and similar anions could be found. ${ }^{112}$ Due to their sensitivity to water and the easy loss of $\mathrm{AlCl}_{3}$, relatively few $\mathrm{AlCl}_{4}^{-}$complexes could be isolated. Addition of $\mathrm{AlCl}_{3}$ to chloro complexes yielded the structurally characterized $\mathrm{Sm}\left(\eta^{6}-\mathrm{C}_{6} \mathrm{Me}_{6}\right)\left(\eta^{2}-\mathrm{Cl}_{2} \mathrm{AlCl}_{2}\right)_{3}, 113 \mathrm{a}, \mathrm{b}$ $\left.\mathrm{U}\left(\eta^{6}-\mathrm{C}_{6} \mathrm{Me}_{6}\right)(\mathrm{ClAlCl})_{3}\right)_{3}, 13 \mathrm{~b}$ [U $\left(\mu_{3}-\mathrm{Cl}\right)_{2}\left(\mu_{2}-\mathrm{Cl}\right)_{3}\left(\mu_{1}-\eta^{2}-\right.$ $\left.\left.\mathrm{Cl}_{2} \mathrm{AlCl}_{2}\right)_{3}\left(\eta^{6}-\mathrm{C}_{6} \mathrm{Me}_{6}\right)\right]\left[\mathrm{AlCl}_{4}\right], 113 \mathrm{c}$ [Pd $\left.\left(\eta^{1}-\mathrm{ClAlCl}\right)_{3}\right)\left(\mu_{2}-\right.$ $\left.\left.\eta^{6}-\mathrm{C}_{6} \mathrm{H}_{6}\right)\right]_{2},{ }^{114}\left(\eta^{6}-\mathrm{C}_{6} \mathrm{R}_{6}\right) \mathrm{Ti}\left(\eta^{2}-\mathrm{Cl}_{2} \mathrm{AlCl}_{2}\right)_{2},{ }^{115 \mathrm{a}}\left(\eta^{3}-\mathrm{C}_{3} \mathrm{H}_{5}\right)-$ $\left[\left(\mathrm{C}_{6} \mathrm{H}_{11}\right)_{3} \mathrm{P}\right] \mathrm{NiClAlMe}{ }_{2} \mathrm{Cl},{ }^{105 c}$ and $\mathrm{Cp}_{2} \mathrm{Ti}(\mathrm{Cl})-$ $\left(\mathrm{ClAlCl}_{2} \mathrm{Me}\right){ }^{115 \mathrm{~b}}$ Other examples are 1:1 adducts of $\mathrm{AlCl}_{3}$ with $\mathrm{Cp}_{2} \mathrm{TiCl}_{2}{ }^{116 \mathrm{a}}$ and $(\mathrm{H})(\mathrm{OC})\left(\mathrm{Ph}_{3} \mathrm{P}\right)_{2} \mathrm{IrCl}_{2} \cdot{ }^{116 \mathrm{~b}}$ An unusual tricoordinate ylide complex [Pt$\left.\left(\mathrm{CH}_{2} \mathrm{PPh}_{3}\right)\left(\mathrm{PPh}_{3}\right) \mathrm{Cl}\right] \mathrm{AlCl}_{4}^{-}$could be obtained by chloride abstraction from $\mathrm{Pt}\left(\mathrm{CH}_{2} \mathrm{PPh}_{3}\right)\left(\mathrm{PPh}_{3}\right) \mathrm{Cl}_{2}$ by $\mathrm{Al}$ $\mathrm{Cl}_{3}{ }^{117}$ Abstraction of a $\mathrm{SiCl}_{3}$ group from $\mathrm{Re}(\mathrm{CO})_{5} \mathrm{SiCl}_{3}$ by $\mathrm{SbCl}_{5}$ produced $\mathrm{Re}(\mathrm{CO})_{5} \mathrm{ClSbCl}_{5}{ }^{118}$ The carboniron bond in $\left[\left(\mathrm{HNC}_{5} \mathrm{H}_{4}\right) \mathrm{CH}_{2} \mathrm{Fe}(\mathrm{CO})_{2} \mathrm{Cp}\right]^{+}$can be cleaved by $\mathrm{Hg}^{2+}$ salts in aqueous solution to give the $\left[\mathrm{CpFe}(\mathrm{CO})_{2}\left(\mathrm{H}_{2} \mathrm{O}\right)\right]^{+}$cation. ${ }^{119}$

Reaction of several Fischer-type carbene complexes with $\mathrm{BF}_{3}$ led to the isolation of $(\mathrm{MeC}) \mathrm{Cr}(\mathrm{CO})_{3}$ $\left(\mathrm{PMe}_{3}\right) \mathrm{FBF}_{3}{ }^{4}$ and $(\mathrm{RC}) \mathrm{W}(\mathrm{CO})_{4} \mathrm{FBF}_{3}{ }^{120} \mathrm{~A}$ niobium hydride was reported to react with $\mathrm{BF}_{3}$ to give a complex of $\mathrm{BF}_{3} \mathrm{H}^{-1}$. 21 Also a general examination of the interaction of metal carbonyl hydrides with Lewis acids $\mathrm{BCl}_{3}$ and $\mathrm{AlBr}_{3}$ was undertaken. ${ }^{122}$ The titanium boranate complex $\mathrm{Cp}_{2} \mathrm{TiBH}_{4}$ and $\mathrm{BF}_{3} \cdot \mathrm{OEt}_{2}$ give $\mathrm{Cp}_{2} \mathrm{TiBF}_{4}$ with bidentate $\mathrm{BF}_{4}-123$

Trialkyl oxonium salts have also been used for the abstraction of halide, ${ }^{24,124}$ methyl, ${ }^{24}$ or azide ${ }^{125 a-c}$ ligands. Halide and pseudohalide abstraction using $\mathrm{R}_{3} \mathrm{O}^{+}$, $\mathrm{SO}_{2} \mathrm{~F}(\mathrm{OMe}), \mathrm{NO}^{+}$, or $\mathrm{BF}_{3}$ is a directed way for the preparation of halide- and pseudohalide-bridged complexes. ${ }^{124-126}\left(\mathrm{C}_{8} \mathrm{H}_{14}\right) \mathrm{AuCl}$ and $\mathrm{SO}_{2} \mathrm{~F}(\mathrm{OMe})$ give the fluorosulfate complex $\left(\mathrm{C}_{8} \mathrm{H}_{14}\right)_{3} \mathrm{AuOSO} \mathrm{F}_{2} \mathrm{~F} .{ }^{127} \mathrm{Cp}_{2} \mathrm{ReH}$ and $\mathrm{AlX}_{3}$ form the hydride-bridged compounds $\mathrm{Cp}_{2} \mathrm{Re}-\mathrm{H}-\mathrm{AlX}_{3},{ }^{128 a}$ while with $\mathrm{CoBr}_{2}$ in $\mathrm{MeCN}$, hydride abstraction and formation of $\left(\mathrm{Cp}_{2} \mathrm{Re}\right)_{2} \mathrm{CoBr}_{4}$ are observed. ${ }^{128 b}$ 


\section{B. Oxidation of Neutral Monomerlc or Dimerlc Complexes}

The oxidation of dimeric metal carbonyls in coordinating solvents has been used to produce cationic complexes of many metals.

$\left[\mathrm{L}_{n} \mathrm{M}(\mathrm{CO})_{m}\right]_{2}+$ solv $\stackrel{-2 \mathrm{e}^{-}}{\longrightarrow} 2\left[\mathrm{~L}_{n} \mathrm{M}(\mathrm{CO})_{m}(\text { solv })\right]^{+}$

A quite general method for the preparation of cationic complexes is oxidation of neutral monomeric and dimeric carbonyl complexes by nitrosyl salts; ${ }^{129}$ e.g., from $\mathrm{M}_{2}(\mathrm{CO})_{10}$ and $\mathrm{NO}^{+}$in $\mathrm{MeCN}$ the cations $\left[(\mathrm{OC})_{5} \mathrm{MNCMe}^{+}(\mathrm{M}=\mathrm{Mn}, \mathrm{Re})\right.$ have been obtained, which are useful starting compounds for other cations $\left[(\mathrm{OC})_{5} \mathrm{ML}\right]^{+} .129 \mathrm{~d}$

Other oxidants that have been used to produce $\left[(\mathrm{OC})_{5} \mathrm{MNCMe}\right]^{+}$are $\mathrm{Cu}^{2+}, \mathrm{Fe}^{3+}$, and TCNE. ${ }^{129 e, \mathrm{f}}$

$\left[\mathrm{CpFe}(\mathrm{CO})_{2}\right]_{2}$ and its derivatives can be oxidized by $\mathrm{Fe}^{3+},{ }^{130} \mathrm{O}_{2}$ in the presence of $\mathrm{HBF}_{4} \cdot \mathrm{OEt}_{2},{ }^{131} \mathrm{Ag}^{+},{ }^{132}$ $\mathrm{Ph}_{3} \mathrm{C}^{+},{ }^{133}$ quinone, ${ }^{134}$ or $\mathrm{Cp}_{2} \mathrm{Fe}^{+135}$ to give [ $\mathrm{CpFe}(\mathrm{CO})-$ $\mathrm{L}$ (solv) $]^{+}$. Similarly, from $\left[\mathrm{CpM}(\mathrm{CO})_{3}\right]_{2}(\mathrm{M}=\mathrm{Cr}, \mathrm{Mo})$ and $\mathrm{Ag}^{+}, 136 \mathrm{Cp}_{2} \mathrm{Fe}^{+}, 135 \mathrm{c}$ or $\mathrm{Ph}_{3} \mathrm{C}^{+}, 137$ [CpRu(CO) $\left.{ }_{2}\right]_{2}$ and $\mathrm{Ag}^{+},{ }^{138}$ and $\left[\mathrm{CpCr}(\mathrm{NO})_{2}\right]_{2}$ and $\mathrm{HBF}_{4} \cdot \mathrm{OEt}_{2}{ }^{139}$ the organometallic Lewis acids $\left[\mathrm{CpM}(\mathrm{CO})_{3}\right]^{+},\left[\mathrm{CpRu}(\mathrm{CO})_{2}\right]^{+}$, and $\left[\mathrm{CpCr}(\mathrm{NO})_{2}\right]^{+}$have been generated in situ. Oxidation of the bis(carbene) complex $\mathrm{Mo}(\mathrm{CO})_{4} \mathrm{~L}_{2}$ with $\mathrm{HO}_{3} \mathrm{SCF}_{3}$ gave the bis(triflato) complex $\mathrm{Mo}(\mathrm{CO})_{2} \mathrm{~L}_{2}(\mathrm{O}$ -

$\left.\mathrm{SO}_{2} \mathrm{CF}_{3}\right)_{2}{ }_{2}^{140}\left(\mathrm{~L}==\mathrm{CN}(\mathrm{Et})\left(\mathrm{CH}_{2}\right)_{2} \mathrm{NEt}\right)$. It should be noted that in the oxidation of the dimeric rhodium complexes $\mathrm{Cp}_{2} \mathrm{Rh}_{2}(\mu-\mathrm{CO})(\mu-\mathrm{L})(\mathrm{L}=\mathrm{CO}$, dppm) with $\mathrm{AgX}$, the cluster compounds $(\mu-\mathrm{AgX})\left[\mathrm{Cp}_{2} \mathrm{Rh}_{2}(\mu-\mathrm{CO})\right.$ $\left(\mu\right.$-L)] are obtained..$^{141,142}$

An elegant way for the synthesis of $\left[\mathrm{CpFe}(\mathrm{CO})_{2-}\right.$ $\left.\left(\mathrm{SO}_{2}\right)\right]^{+},\left[\mathrm{Mn}(\mathrm{CO})_{5}\left(\mathrm{SO}_{2}\right)\right]^{+},\left[\operatorname{Re}(\mathrm{CO})_{5}\left(\mathrm{SO}_{2}\right)\right]^{+}$, and [Co$\left.(\mathrm{CO})_{4}\left(\mathrm{SO}_{2}\right)\right]^{+}$is the oxidation of the corresponding neutral dimers with $\mathrm{AsF}_{5}$ in liquid $\mathrm{SO}_{2} ;{ }^{143}$ e.g.

$$
\begin{aligned}
& \operatorname{Re}_{2}(\mathrm{CO})_{10}+3 \mathrm{AsF}_{5}+2 \mathrm{SO}_{2} \rightarrow \\
& 2\left[\operatorname{Re}(\mathrm{CO})_{5}\left(\mathrm{SO}_{2}\right)\right]^{+} \mathrm{AsF}_{6}^{-}+\mathrm{AsF}_{3}
\end{aligned}
$$

Another interesting example is $\operatorname{Re}(\mathrm{CO})_{5} \mathrm{FReF}_{5}$, which can be obtained from $\operatorname{Re}_{2}(C O)_{10}$ and $\mathrm{ReF}_{6}$ or $\mathrm{XeF}_{2}$, respectively..$^{144}$

The hydrido complex $\mathrm{CpFeL}_{2} \mathrm{H}$ and $\left[\mathrm{CpFe}(\mathrm{CO})_{2} \mathrm{~L}^{\prime \prime}\right]^{+}$ $\left(\mathrm{L}^{\prime \prime}=\right.$ olefin, carbene) undergo redox reactions to give $\left[\mathrm{CpFeL}_{2} \text { (solv) }\right]^{+}$and $\mathrm{CpFe}(\mathrm{CO})_{2}$ (alkyl). ${ }^{145} \mathrm{Cp}_{2} \mathrm{ReH}$ is oxidized by $\mathrm{CuCl}_{2}$ to $\left[\mathrm{Cp}_{2} \mathrm{Re}\right]^{+}\left[\mathrm{CuCl}_{2}^{-}\right]$. $^{146}$

Examples of dicationic complexes obtained by the oxidation of monomeric complexes include [Pd$\left.\left(\mathrm{PPh}_{3}\right)_{4}\right]^{2+}$ (from $\mathrm{Pd}\left(\mathrm{PPh}_{3}\right)_{4}$ and $\left.\mathrm{Ph}_{3} \mathrm{C}^{+}\right)$, ${ }^{147}\left[\mathrm{MoH}_{2}-\right.$ $\left.\left(\mathrm{PR}_{3}\right)_{4}(\mathrm{NCMe})_{2}\right]^{2+}$ (from $\mathrm{MoH}_{4}\left(\mathrm{PR}_{3}\right)_{4}$ and $\left.\mathrm{AgBF}_{4}\right)^{78}$ $\left[\mathrm{CpCoL}_{2}\right]^{2+}$ (from $\mathrm{CpCoL}_{2}$ and $\left.\mathrm{AgBF}_{4}\right),{ }^{148}\left[\mathrm{CpCoL}_{3}\right]^{2+}$ (from $\mathrm{CpCo}(\mathrm{CO})_{2}, \mathrm{~L}$, and $\left.\left[\mathrm{Cp}_{2} \mathrm{Fe}\right]^{+} \mathrm{BF}_{4}^{-}\right),{ }^{149}\left[\mathrm{M}(\mathrm{NO})_{2}-\right.$ $\left.\left(\mathrm{NCCH}_{3}\right)_{4}\right]^{2+}$ (from $\mathrm{M}(\mathrm{CO})_{6}(\mathrm{M}=\mathrm{Mo}, \mathrm{W})$ and $\left.\mathrm{NOBF}_{4}\right){ }^{150}$ and $\left[\mathrm{Mo}(\mathrm{CO})_{2}(\mathrm{bpy})_{2}\left(\mathrm{OH}_{2}\right)\right]^{2+}\left(\mathrm{BF}_{4}{ }^{-}\right)_{2}$ (from $\mathrm{Mo}(\mathrm{CO})_{2}(\mathrm{bpy})_{2}$ and $\left.\mathrm{AgBF}_{4}\right){ }_{151}$

A report of the synthesis of $\left[\mathrm{Mo}(\mathrm{NO})_{5}\right]^{5+}\left[\mathrm{BF}_{4}{ }^{-}\right]_{5}$ from $\mathrm{Mo}(\mathrm{CO})_{6}$ and $\mathrm{NOBF}_{4}$ in $\mathrm{Cl}_{2} \mathrm{C}_{2} \mathrm{H}_{4}$ is highly surprising ${ }^{152}$ and certainly wrong. Reactions of $\mathrm{M}(\mathrm{CO})_{6}$ with NOX $\left(\mathrm{X}=\mathrm{Cl}, \mathrm{PF}_{6}\right)$ give polymeric $\left[\mathrm{M}(\mathrm{NO})_{2} \mathrm{X}_{2}\right]_{n} \cdot{ }^{153,185}$ " $\left[\mathrm{Mo}(\mathrm{NO})_{5}\right]^{5+}\left[\mathrm{BF}_{4}^{-}\right]_{5}$ " and $\mathrm{Mo}(\mathrm{NO})_{2}\left(\mathrm{PF}_{6}\right)_{2}{ }^{186}$ show identical $\nu$ (NO) bands.

Seventeen-electron species can also be obtained ${ }^{148,154}$ by oxidation of 18-electron complexes, e.g., [CpFe(dppe)I] ${ }^{+} \mathrm{PF}_{6}{ }^{-155}$ In the electrochemical oxidation of
$\left[\mathrm{M}(\mathrm{CO})_{3} \mathrm{Cp}\right]_{2}$ and $\mathrm{Mn}_{2}(\mathrm{CO})_{10}$ the radicals $\left[\mathrm{M}(\mathrm{CO})_{3} \mathrm{Cp}\right]^{\cdot}$ and $\left[\mathrm{Mn}(\mathrm{CO})_{5}\right]^{\circ}$ are key intermediates. ${ }^{156,157}$ Electrochemical preparations of cationic complexes include $\left[\mathrm{CpFe}(\mathrm{CO})_{2}(\mathrm{solv})\right]^{+},{ }^{118}\left[\mathrm{IrR}(\mathrm{CO})\left(\mathrm{PPh}_{3}\right)_{3}\right]^{+159}[\mathrm{CpRh}-$ (CO) $\left.\left(\mathrm{PPh}_{3}\right)\right]^{+},{ }_{160}$ and $\left[\mathrm{CpCr}(\mathrm{NO})_{2} \text { (solv) }\right]^{+} .161$

Recently, a complex of coordinated $\mathrm{SbF}_{6}{ }^{-}$could be prepared by oxidation of $\mathrm{W}(\mathrm{CO})_{5}\left(\mathrm{PPh}_{3}\right)$ with $\mathrm{NOSbF}_{6}: 162$

$\mathrm{W}(\mathrm{CO})_{5}\left(\mathrm{PR}_{3}\right)+\mathrm{NOSbF}_{6} \underset{\mathrm{W}(\mathrm{CO})_{3}(\mathrm{NO})\left(\mathrm{PR}_{3}\right)}{\stackrel{\mathrm{CH}_{2} \mathrm{Cl}_{2}}{\longrightarrow}}\left(\mathrm{FSbF}_{5}\right)+2 \mathrm{CO}$

\section{Oxidative Addition of RX to Metal $d^{8}$ Systems}

The oxidative addition of strong acids, of alkyl fluorosulfates, or of oxonium salts to Vaska's compound $\operatorname{IrCl}(\mathrm{CO})\left(\mathrm{PPh}_{3}\right)_{2}$ gives the complexes $\operatorname{Ir}(\mathrm{CO})(\mathrm{Cl})$ $\left(\mathrm{PPh}_{3}\right)_{2}(\mathrm{R})(\mathrm{X})$ (where $\mathrm{R}=\mathrm{H}$ or $\mathrm{Me}$ and $\mathrm{X}=\mathrm{FBF}_{3}$, $\mathrm{FPF}_{5}, \mathrm{OPOF}_{2}, \mathrm{OSO}_{2} \mathrm{CF}_{3}, \mathrm{OSO}_{2} \mathrm{C}_{4} \mathrm{~F}_{9}, \mathrm{OSO}_{2} \mathrm{~F}$, or $\left.\mathrm{OClO}_{3}\right)$. ${ }^{14 a, b, 163,164}$ Similarly, $\operatorname{Ir}\left(\mathrm{N}_{2}\right)\left(\mathrm{PPh}_{3}\right)_{2} \mathrm{Cl}$ and RX give $\operatorname{Ir}\left(\mathrm{N}_{2}\right)\left(\mathrm{PPh}_{3}\right)_{2}(\mathrm{Cl})(\mathrm{R})(\mathrm{X})\left(\mathrm{R}=\mathrm{H}, \mathrm{Me} ; \mathrm{X}=\mathrm{OSO}_{2} \mathrm{~F}\right.$, $\left.\mathrm{OSO}_{2} \mathrm{CF}_{3}, \mathrm{FBF}_{3}\right),{ }^{163,165}$ which have two good leaving groups, similar to the above-mentioned $\left[\mathrm{CpMo}(\mathrm{CO})_{2}-\right.$ $\left.\left(\mathrm{CH}_{2}=\mathrm{CHMe}\right)\left(\mathrm{FBF}_{3}\right)\right]_{.}{ }^{104}$ The Brönsted acidity of strong acids $\mathrm{HX}$ is transferred to Lewis acidity at the metal by these reactions. Reacting $\mathrm{Pt}\left(\mathrm{PPh}_{3}\right)_{2}\left(\mathrm{C}_{2} \mathrm{H}_{4}\right)$ with $\mathrm{HBF}_{4}$ or $\mathrm{HSO}_{3} \mathrm{CF}_{3}$ gives $\mathrm{Pt}\left(\mathrm{PPh}_{3}\right)_{2} \mathrm{H}_{2} \mathrm{X}_{2}\left(\mathrm{X}=\mathrm{BF}_{4}\right.$, $\left.\mathrm{CF}_{3} \mathrm{SO}_{3}\right]_{166}$ and addition of $\mathrm{H}_{2} \mathrm{C}\left(\mathrm{SO}_{2} \mathrm{CF}_{3}\right)_{2}$ yields $\left(\mathrm{PPh}_{3}\right)_{2} \mathrm{PtH}\left[\mathrm{HC}\left(\mathrm{SO}_{2} \mathrm{CF}_{3}\right)_{2}\right] .{ }^{167} \mathrm{Pt}\left(\mathrm{PPh}_{3}\right)_{2} \mathrm{Cl}_{2}$ adds two molecules of $\mathrm{RX}$ to give $\mathrm{Pt}\left(\mathrm{PPh}_{3}\right)_{2} \mathrm{HClX}_{2}{ }^{166}$ or $\left[\mathrm{Pt}_{2}-\right.$ $\left.\left(\mathrm{PPh}_{3}\right)_{4} \mathrm{Cl}_{2}\right]\left[\mathrm{FSO}_{3}\right]_{2}{ }^{1246}$ depending on the nature of $\mathrm{RX}$. Addition of $\mathrm{HBF}_{4}$ to the dinuclear complex $[\mathrm{RhCl}(\mu$ dppm $]_{2}\left(\mu\right.$-CO) gives the mixed $\mathrm{Rh}^{\mathrm{I}} / \mathrm{Rh}^{\mathrm{III}}$ dimer $\left[\mathrm{RhCl}(\mu-\mathrm{dppm})_{2}\right]_{2}(\mu-\mathrm{CO})(\mu-\mathrm{H})\left(\mathrm{FBF}_{3}\right){ }^{39}$

\section{Miscellaneous}

Photochemical-induced disproportionation of dimeric complexes $\left[(\mathrm{MeCp}) \mathrm{Mo}(\mathrm{CO})_{3}\right]_{2}$ to give $[(\mathrm{MeCp}) \mathrm{Mo}$ $\left.(\mathrm{CO})_{3}(\mathrm{solv})\right]^{+}$and $\left[(\mathrm{MeCp}) \mathrm{Mo}(\mathrm{CO})_{3}\right]^{-}$has been reported. ${ }^{168}$ The highly reactive "[ $\left.\mathrm{CpFe}(\mathrm{dppe})\right]^{+\infty}$ is formed by irradiation of $[\mathrm{CpFe}(\mathrm{CO}) \text { (dppe) }]^{+}$; it coordinates a large variety of ligands. ${ }^{169}$ Abstraction of $\mathrm{Cl}^{-}$ from $\mathrm{CpCr}(\mathrm{NO})_{2} \mathrm{CH}_{2} \mathrm{Cl}$ by $\mathrm{Ag}^{+}$leads via the intermediate $\left[\mathrm{CpCr}(\mathrm{NO})_{2}=\mathrm{CH}_{2}\right]^{+}$to ${ }^{\circ}\left(\mathrm{C}_{5} \mathrm{H}_{4} \mathrm{CH}_{3}\right) \mathrm{Cr}(\mathrm{NO})_{2}{ }^{+\cdots} \cdot{ }^{170}$ The disporportionation of cationic methylene complexes gives the intermediate organometallic Lewis acids and cationic ethylene compounds: ${ }^{90,171-173}$

$$
2\left[\mathrm{~L}_{n} \mathrm{M}=\mathrm{CH}_{2}\right]^{+} \rightarrow\left[\mathrm{L}_{n} \mathrm{M}\left(\mathrm{C}_{2} \mathrm{H}_{4}\right)\right]^{+}+{ }^{-} \mathrm{L}_{n} \mathrm{M}^{+”}
$$

where $\mathrm{L}_{n} \mathrm{M}=\mathrm{CpL}_{2} \mathrm{Fe},{ }^{171} \mathrm{Cp}(\mathrm{OC})_{2} \mathrm{LMo}, \mathrm{Cp}$ $(\mathrm{OC})_{2} \mathrm{LW},{ }^{90,172}$ or $\mathrm{Cp}(\mathrm{ON})\left(\mathrm{Ph}_{3} \mathrm{P}\right) \mathrm{Re}^{173}\left(\mathrm{~L}=\mathrm{CO}, \mathrm{PR}_{3}\right)$. Triflato complexes can be isolated by this route. ${ }^{90,171 d, \theta}$ An unusual weak ligand is $\mathrm{V}(\mathrm{CO})_{6}{ }^{-}$in $\mathrm{V}(\mathrm{THF})_{4}[(\mu-$ OC) $\left.\mathrm{V}(\mathrm{CO})_{5}\right]_{2}{ }^{174}$ and $\left(\mathrm{C}_{5} \mathrm{Me}_{5}\right)_{2} \mathrm{~V}(\mu-\mathrm{OC}) \mathrm{V}(\mathrm{CO})_{5},{ }^{175}$ which is coordinated via the oxygen atom of one carbonyl ligand to the Lewis acid [V(THF $\left.)_{4}\right]^{2+}$ and $\left[\left(\mathrm{C}_{5} \mathrm{Me}_{5}\right)_{2} \mathrm{~V}\right]^{+}$, respectively. In the latter complex $\mathrm{V}(\mathrm{CO})_{6}{ }^{-}$is easily displaced by nitrogen donors. ${ }^{175}$

\section{Proof of Coordination}

In Table I a listing of isolated and characterized complexes of coordinated fluoro anions, $\mathrm{OTeF}_{5}^{-}$, and carborane anions is given. 
TABLE I. Representative Examples of Proposed or Isolated Complexes with Fluoro-, Teflate- and Carborane anions

\begin{tabular}{|c|c|c|}
\hline complex & $\mathrm{X}, \mathrm{L}$ & ref \\
\hline $\begin{array}{l}\mathrm{CrCp}(\mathrm{NO})_{2} \mathrm{X} \\
\mathrm{Cr}(\mathrm{CO})_{3}(\mathrm{CMe})\left(\mathrm{PMe}_{3}\right) \mathrm{X} \\
\mathrm{MoCp}(\mathrm{CO})_{2}(\mathrm{~L}) \mathrm{X} \\
\mathrm{WCp}(\mathrm{CO})_{2}(\mathrm{~L}) \mathrm{X} \\
\mathrm{WCp}(\mathrm{NO})_{2} \mathrm{X} \\
\mathrm{W}(\mathrm{CO})_{3}(\mathrm{NO})\left(\mathrm{PR}_{3}\right) \mathrm{X} \\
\mathrm{Mn}(\mathrm{CO})_{5} \mathrm{X} \\
\operatorname{Re}(\mathrm{CO})_{3}(\mathrm{~L})_{2} \mathrm{X} \\
\operatorname{Re}(\mathrm{Cp})(\mathrm{NO})\left(\mathrm{PPh}_{3}\right) \mathrm{X} \\
\mathrm{FeCp}(\mathrm{CO})_{2} \mathrm{X} \\
\mathrm{Fe}(\mathrm{TPP}) \mathrm{X} \\
{\left[\mathrm{Rh}_{2} \mathrm{Cl} \mathrm{Cl}_{2}(\mu-\mathrm{H})(\mu-\mathrm{CO})(\mathrm{dppm})_{2} \mathrm{X}\right]} \\
\operatorname{Ir}(\mathrm{L})(\mathrm{Cl})(\mathrm{PPh})_{2}(\mathrm{H}) \mathrm{X} \\
\mathrm{U}_{2}\left(\mathrm{C}_{6} \mathrm{H}_{3}\left(\mathrm{SiMe}_{3}\right)_{2}\right)_{4}(\mu-\mathrm{F})_{2}(\mu-\mathrm{X})_{2} \\
\operatorname{trans}_{2}\left(\mathrm{Ph}_{3} \mathrm{P}\right)_{2} \mathrm{PtHX}\end{array}$ & 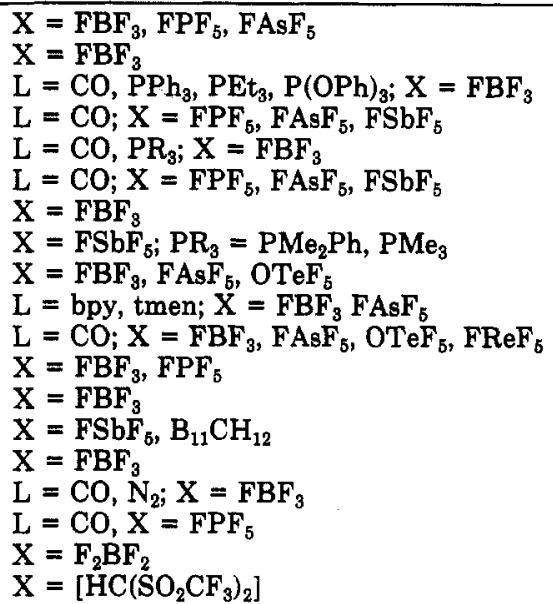 & $\begin{array}{l}20,60,161 \\
4 \\
5,182 \\
5,25 \\
5,186 \mathrm{a} \\
5,202 \\
222 \\
162 \\
3,54,183 \\
56 \mathrm{c} \\
3,42,54,88,144 \\
91 \mathrm{~b} \\
53 \\
179,180 \\
39 \\
164 \\
252 \\
176 \\
167\end{array}$ \\
\hline
\end{tabular}

TABLE II. Bond Lengths in Some M-F-EF Complexes $_{n}$

\begin{tabular}{lllll}
\hline \multicolumn{1}{c}{ compd } & $d\left(\mathrm{M}-\mathrm{F}_{\mathrm{b}}\right)$ & \multicolumn{1}{c}{$d\left(\mathrm{~F}_{\mathrm{b}}-\mathrm{E}\right)$} & $d\left(\mathrm{E}-\mathrm{F}_{\mathrm{t}}\right)$ & ref \\
\hline $\mathrm{Cu}\left(\mathrm{PPh}_{3}\right)_{3} \mathrm{FBF}_{3}$ & 2.31 & 1.39 & 1.35 & $13 \mathrm{c}$ \\
{$\left[\mathrm{UCp}{ }_{2}\left(\mu-\mathrm{BF}_{4}\right)(\mu-\mathrm{F})\right]_{2}$} & 2.41 & 1.34 & $1.38 / 1.23$ & 176 \\
{$\left[\mathrm{Cu}\left(\mathrm{bpyy}_{2}\left(\mathrm{~F}_{2} \mathrm{BF}_{2}\right)\right]^{+\mathrm{BF}_{4}-}\right.$} & $2.56 / 2.66$ & $1.37 / 1.35$ & 1.39 & $14 \mathrm{a}$ \\
$\mathrm{IrClCO}\left(\mathrm{PPh}_{3}\right)_{2}(\mathrm{H})\left(\mathrm{FBF}_{3}\right)$ & 2.27 & 1.45 & 1.34 & $164 \mathrm{~b}$ \\
$\mathrm{Ag}(\mathrm{CNR})_{2} \mathrm{~F}_{2} \mathrm{PF}_{4}$ & 2.67 & 1.56 & 1.51 & 178 \\
$\mathrm{Fe}(\mathrm{TPP}) \mathrm{FSbF}_{5}$ & 2.11 & 1.90 & $1.88-1.93$ & $180 \mathrm{a}$ \\
$\left(\mathrm{Me} \mathrm{PhP}_{3}\left(\mathrm{CO}_{2}\right)_{2} \mathrm{ONWFSbF}_{5}\right.$ & 2.17 & 1.95 & 1.86 & 162 \\
$(\mathrm{OC})_{5} \mathrm{ReFReF}$ & & $1.98 / 1.95$ & 1.84 & 144
\end{tabular}

TABLE III. $\nu(E-F)$ Bands $\left(\mathrm{cm}^{-1}\right)$ of Hexafluorophosphato, -arsenato, and -antimonato Complexes $\mathrm{L}_{n} \mathbf{M}^{-X^{a}}$

\begin{tabular}{|c|c|c|c|c|c|c|c|c|c|}
\hline $\mathrm{L}_{n} \mathrm{M}$ & $\mathrm{X}$ & $\mathrm{A}_{1}\left(\nu_{1}\right)$ & $A_{1}\left(\nu_{2}\right)$ & $\mathbf{B}_{1}\left(\nu_{2}\right)$ & $A_{1}\left(\nu_{3}\right)$ & $\mathrm{E}\left(\nu_{3}\right)$ & $A_{1}\left(\nu_{4}\right)$ & $\mathrm{E}\left(\nu_{4}\right)$ & ref \\
\hline $\begin{array}{l}\mathrm{Cu}(\mathrm{py})_{4} \\
\mathrm{CpMo}(\mathrm{CO})_{3} \\
\mathrm{IrHCl}\left(\mathrm{PPh}_{3}\right)_{2} \mathrm{CO} \\
\mathrm{CpMo}(\mathrm{CO})_{3} \\
\mathrm{CpW}(\mathrm{CO})_{3} \\
\mathrm{Re}(\mathrm{CO})_{5} \\
\mathrm{Mn}(\mathrm{CO})_{5} \\
\operatorname{Re}(\mathrm{CO})_{3}(\mathrm{bpy}) \\
\mathrm{CpMo}(\mathrm{CO})_{3}\end{array}$ & $\begin{array}{l}\left(\mathrm{FPF}_{5}\right)_{2} \\
\mathrm{FPF}_{5} \\
\mathrm{FPF}_{5} \\
\mathrm{FAsF}_{5} \\
\text { FAsF }_{5} \\
\text { FAsF }_{5} \\
\text { FAsF }_{5} \\
\text { FAsF }_{5} \\
\text { FSbF }_{5}\end{array}$ & $\begin{array}{l}742 \\
739 \\
734 \\
669 \\
668 \\
675 \\
676 \\
\\
642\end{array}$ & 511 & 550 & $\begin{array}{l}852 \\
879 \\
880 \\
699 \\
697 \\
712 \\
710 \\
705 \\
662\end{array}$ & $\begin{array}{l}828 \\
810 \\
810 \\
726 \\
726 \\
730 \\
725 \\
765 \\
679\end{array}$ & $395,384,367$ & $\begin{array}{l}551 \\
488 \\
488\end{array}$ & $\begin{array}{l}181 \\
5 \\
116 \mathrm{~b} \\
25 \\
34 \\
3 \\
3 \\
56 \mathrm{c} \\
25\end{array}$ \\
\hline
\end{tabular}

${ }^{-} \mathrm{The} \nu(\mathrm{E}-\mathrm{F})$ bands $\left(\mathrm{F}_{\mathrm{lu}}\right)$ of the free octahedral anions are at ca. $830 \mathrm{~cm}^{-1}\left(\mathrm{PF}_{6}^{-}\right), 700 \mathrm{~cm}^{-1}\left(\mathrm{AsF}_{6}^{-}\right)$, and $670 \mathrm{~cm}^{-1}\left(\mathrm{SbF}_{6}{ }^{-}\right)$.

\section{A. Crystal Structure Determinatlons}

A series of crystal structures of non-carbonyl complexes show $\mathrm{BF}_{4}^{-}$as a monodentate or bidentate bridging ligand. 13,14,176,177 Also the structures of complexes with $\mathrm{PF}_{6}^{-178} \mathrm{AlCl}_{4}^{-}$, and $\mathrm{AlCl}_{2} \mathrm{R}_{2}^{-105 c, 113-115}$ have been elucidated. A group of $\mathrm{Fe}(\mathrm{TPP}) \mathrm{X}$ complexes, particularly with the weak anionic ligands $\mathrm{X}=\mathrm{SbF}_{6}{ }^{-179}$ and $\mathrm{B}_{11} \mathrm{CH}_{12}{ }^{-180}$ was examined by $\mathrm{X}$-ray crystallography. The crystal structure of $\mathrm{AgB}_{11} \mathrm{CH}_{12} \cdot 2 \mathrm{C}_{6} \mathrm{H}_{6}{ }^{180 \mathrm{c}}$ shows a bridging carborane and an $\eta^{1}$-coordinated benzene ligand, which is "interpreted as resulting from the poor ligation properties of the closo-carborane anion". Only a few crystal structures of carbonyl complexes with $\mathrm{BF}_{4}^{-}, \mathrm{SbF}_{6}{ }^{-}, \mathrm{ReF}_{6}{ }^{-}$, and $\mathrm{OTeF}_{5}{ }^{-}$have been reported, e.g., $\operatorname{IrCl}(\mathrm{CO})\left(\mathrm{PPh}_{3}\right)_{2}(\mathrm{H})\left(\mathrm{FBF}_{3}\right){ }_{1,164}$ $\left(\mathrm{Me}_{2} \mathrm{PhP}\right)(\mathrm{CO})_{3}(\mathrm{NO}) \mathrm{WFSbF}_{5},{ }^{162}(\mathrm{OC})_{5} \mathrm{ReFReF}_{5},{ }^{144}$ and $(\mathrm{OC})_{5} \mathrm{MnOTeF}_{5}{ }^{54}$ In most of these compounds the bridging E-F bonds are longer than the terminal E-F bonds. The metal-fluorine interaction is stronger in complexes of $\pi$-acceptor ligands than it is in complexes of metals in normal oxidation states with nitrogen donors. The two very different terminal $B-F$ bond lengths in $\left[\mathrm{UCp}_{2}^{\prime \prime}{ }_{2}\left(\mu-\mathrm{BF}_{4}\right)(\mu-\mathrm{F})_{2}\right]\left(\mathrm{Cp}^{\prime \prime}=\mathrm{C}_{5} \mathrm{H}_{3}\left(\mathrm{SiMe}_{3}\right)_{2}\right)$ are unusual. ${ }^{176}$
The coordination of $\mathrm{CH}\left(\mathrm{SO}_{2} \mathrm{CF}_{3}\right)_{2}{ }^{-}$to platinum via the carbon atom was shown in a crystal structure determination of $\mathrm{Pt}\left(\mathrm{PPh}_{3}\right)_{2}(\mathrm{H})\left(\mathrm{CH}\left(\mathrm{SO}_{2} \mathrm{CF}_{3}\right)_{2}\right) .{ }^{167 \mathrm{~b}}$ This appears interesting since $\mathrm{RHC}\left(\mathrm{SO}_{2} \mathrm{CF}_{3}\right)_{2}$ are strong protic acids and the negative charge of the corresponding anion should be delocalized.

Bond lengths of relevant examples are summarized in Table II.

\section{B. IR Spectroscopy}

Coordination of the highly symmetric anions $\mathrm{BF}_{4}^{-}$ $\left(T_{d}\right), \mathrm{PF}_{6}^{-}, \mathrm{AsF}_{6}^{-}$, or $\mathrm{SbF}_{6}^{-}\left(O_{h}\right)$ to a metal center leads to a significant lowering of the symmetry. This results in a characteristic splitting of the E-F stretching vibrations ${ }^{13 \mathrm{c}, 181}$ (Table III). The $\nu\left(\mathrm{BF}_{4}\right)$ vibrations are especially sensitive to changes in the neighborhood of the $\mathrm{BF}_{4}^{-}$anion. Four $\nu(\mathrm{BF})$ bands are expected for the $C_{s}$ symmetry of the $\mathrm{M}-\mathrm{F}-\mathrm{BF}_{3}$ group, as has been observed with $\mathrm{M}(\mathrm{CO})_{5} \mathrm{FBF}_{3}(\mathrm{M}=\mathrm{Mn}, \mathrm{Re})$; sometimes only three bands are visible (Table IV).

Splitting of the $v\left(\mathrm{BF}_{4}\right)$ band may also be observed without coordination of the anions to a metal center, due to hydrogen bonds to coordinated water or amines. $4,56 c, 184$ 
TABLE IV. $\nu\left({ }^{11} \mathrm{~B}-\mathrm{F}\right)$ Bands of Tetrafluoroborato Complexes ${ }^{a}$

\begin{tabular}{llrrll}
\hline \multicolumn{1}{c}{ compd } & \multicolumn{3}{c}{$\nu\left({ }^{11} \mathrm{~B}-\mathrm{F}\right)$} & ref \\
\cline { 2 - 5 } $\mathrm{CpMo}(\mathrm{CO})_{3} \mathrm{FBF}_{3}$ & 1130 & & 884 & 722 & 5 \\
$\mathrm{CpMo}(\mathrm{CO})_{2}\left(\mathrm{PPh}_{3}\right) \mathrm{FBF}_{3}$ & 1119 & & 901 & 732 & 182 \\
$\mathrm{CpMo}(\mathrm{CO})_{2}\left(\mathrm{PEt}_{3}\right) \mathrm{FBF}_{3}$ & 1137 & & 891 & 730 & 182 \\
$\left.\mathrm{Cu}(\mathrm{PPh})_{3}\right)_{3} \mathrm{FBF}_{3}$ & & 984 & 769 & & $13 \mathrm{c}$ \\
$\mathrm{Cr}(\mathrm{CMe})\left(\mathrm{CO}_{3}\left(\mathrm{PMe}_{3}\right) \mathrm{FBF}_{3}\right.$ & 1110 & & 910 & 745 & 4 \\
$\mathrm{Re}(\mathrm{CO})_{5} \mathrm{FBF}_{3}$ & 1162 & 1128 & 902 & 738 & 42 \\
$\mathrm{Mn}(\mathrm{CO})_{5} \mathrm{FBF}_{3}$ & 1145 & 1118 & 919 & 738 & 183 \\
{$\left[\mathrm{Cu}(\mathrm{bpy})_{2} \mathrm{~F}_{2} \mathrm{BF}_{3}{ }^{+} \mathrm{BF}_{4}^{-}\right.$} & 1105 & 1070 & 1030 & & $14 \mathrm{a}$ \\
{$\left[\mathrm{SnMe} \mathrm{F}_{2} \mathrm{BF}_{2}\right]^{+} \mathrm{BF}_{4}^{-}$} & 1170 & 1085 & 1005 & 758 & $14 \mathrm{a}$
\end{tabular}

a The free $\mathrm{BF}_{4}^{-}$ion absorbs at ca. $1050-1080 \mathrm{~cm}^{-1}$.

We have found that some very labile complexes can react with the material of the IR cell windows rapidly under formation of halide compounds. Then only the IR spectrum of the free anions is observed. In other cases the presence of water leads also to splitting of $\nu\left(\mathrm{BF}_{4}\right)$ bands (see below).

Evidence for bridging $\mathrm{F}_{2} \mathrm{PF}_{4}$ groups in polymeric $\left[\mathrm{Mo}(\mathrm{NO})_{2}\left(\mathrm{PF}_{6}\right)_{2}\right]_{n}$ has been obtained by IR spectroscopy. ${ }^{185}$

\section{NMR Spectroscopy}

In a few cases it has been possible to demonstrate the coordination by NMR spectroscopy. The ${ }^{19} \mathrm{~F}$ NMR low-temperature spectra of $\mathrm{CpM}(\mathrm{CO})_{2}(\mathrm{~L})\left(\mathrm{FBF}_{3}\right)^{186}$ in $\mathrm{CD}_{2} \mathrm{Cl}_{2}$ show two widely separated signals; a doublet due to the terminal fluorine nuclei close to free $\mathrm{BF}_{4}^{-}$ and a quartet at very high field due to the bridging fluorine atom. Similarly, coordination of $\mathrm{BF}_{4}^{-}$in $\mathrm{CpFe}\left(\mathrm{C}_{6} \mathrm{H}_{8}\right)\left(\mathrm{FBF}_{3}\right)$ was proven by ${ }^{19} \mathrm{~F}$ NMR spectroscopy. ${ }^{103}$ In phosphorus-containing compounds ( $\mathrm{L}=$ $\mathrm{PR}_{3}$ ) additional coupling to the ${ }^{31} \mathrm{P}$ nuclei can be observed. ${ }^{186,187}$ The ${ }^{31} \mathrm{P}$ NMR spectra of these complexes as well of $\left(\mathrm{MeC} \equiv \mathrm{Cr}(\mathrm{CO})_{3}\left(\mathrm{PMe}_{3}\right) \mathrm{FBF}_{3},{ }_{4} \mathrm{~W}(\mathrm{CO})_{3}\right.$ $\left(\mathrm{PR}_{3}\right)(\mathrm{NO})\left(\mathrm{FSbF}_{5}\right){ }^{162}$ and $\left(\eta^{3}-\mathrm{C}_{3} \mathrm{H}_{5}\right)\left(\mathrm{Me}_{3} \mathrm{P}\right) \mathrm{NiFBF}_{3}{ }^{105 \mathrm{a}}$ also reveal a doublet at low temperature due to coupling of the ${ }^{31} \mathrm{P}$ nucleus to a single fluorine atom. At higher temperatures typical coalescence behavior in both ${ }^{31} \mathrm{P}$ and ${ }^{19} \mathrm{~F}$ NMR spectra is observed, which is consistent with an intramolecular exchange of coordinated and noncoordinated fluorine atoms.

In some cases only signals of freely rotating or uncoordinated fluoro anions could be obtained. ${ }^{30,56 c, 60,91 b, 186 a}$ This was explained by an equilibrium between the coordinated and free $\mathrm{BF}_{4}{ }^{-}$anion ${ }^{56 \mathrm{c}}$ or by rapid rotation of $\mathrm{PF}_{6}{ }^{-}$on the NMR time scale; ${ }^{60}$ a fast exchange of solvent and coordinated $\mathrm{PF}_{6}{ }^{-}$may also be an explanation. ${ }^{186 \mathrm{~B}}$ For $\mathrm{CpRe}(\mathrm{NO})\left(\mathrm{PPh}_{3}\right) \mathrm{BF}_{4}{ }^{13} \mathrm{C}$ NMR evidence of coordinated $\mathrm{CD}_{2} \mathrm{Cl}_{2}$ in $\mathrm{CD}_{2} \mathrm{Cl}_{2}$ solution has been obtained. ${ }^{91 \mathrm{~b}}$ The relatively small number of NMR "proofs" is a consequence of the low solubility of many of these complexes in weakly coordinating solvents. In stronger donor solvents substitution of the coordinated anion by the solvent occurs.

\section{Reactivity}

Organometallic Lewis acids such as " $\operatorname{Re}(\mathrm{CO})_{5}{ }^{+"}$ or " $\mathrm{AuPPh}{ }_{3}{ }^{+"}$ can serve as a probe for the behavior of all kinds of ligands, including ambivalent ligands. "Re$(\mathrm{CO})_{5}{ }^{+}$is especially useful since the pentacarbonyl group can easily be detected by its characteristic pattern of the $\nu(\mathrm{CO})$ bands in the IR spectrum.
TABLE V

A. CO Frequencies $\left(\mathrm{cm}^{-1}\right)$ of Carbonyl Complexes $\mathrm{L}_{n}(\mathrm{CO})_{n} \mathrm{MX}$ of Weakly Weakly Coordinating Anions

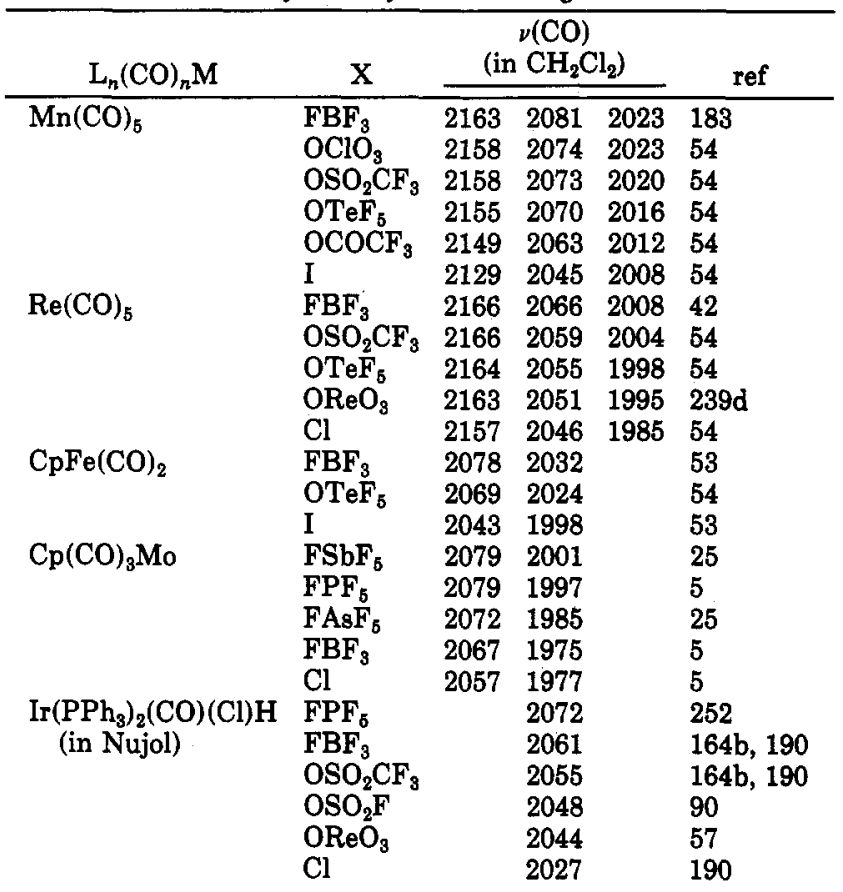

B. NO Frequencies $\left(\mathrm{cm}^{-1}\right)$ of Complexes $\mathrm{CpW}(\mathrm{NO})_{2} \mathrm{X}$

\begin{tabular}{llll}
\hline $\mathrm{X}$ & \multicolumn{2}{c}{$\nu(\mathrm{NO})$} & ref \\
\hline FBF & 1770 & 1640 & 222 \\
OTs & 1737 & 1650 & 222 \\
Cl & 1705 & 1625 & 222
\end{tabular}

TABLE VI. $\delta\left(\mathrm{C}_{5} \mathrm{H}_{5}\right)$ Signals of Several Cyclopentadienyl Complexes $\mathrm{CpL}_{n} \mathrm{MX}$ of Weakly Coordinated Anions

\begin{tabular}{clcl}
\hline $\mathrm{CpL}_{n} \mathrm{M}$ & \multicolumn{1}{c}{$\mathrm{X}$} & $\delta\left(\mathrm{C}_{5} \mathrm{H}_{5}\right)^{a}$ & ref \\
\hline $\mathrm{Cp}(\mathrm{CO})_{3} \mathrm{Mo}$ & $\mathrm{FSbF}_{5}$ & 6.02 & 25 \\
& $\mathrm{FAsF}_{5}$ & 6.02 & 25 \\
& $\mathrm{FBF}_{3}$ & 5.98 & 90 \\
& $\mathrm{OSO}_{2} \mathrm{~F}$ & 5.88 & 90 \\
& $\mathrm{OSO}_{2} \mathrm{CF}_{3}$ & $5.85^{b}$ & 90 \\
$\mathrm{Cl}$ & 5.70 & 5 \\
$\mathrm{Cp}(\mathrm{CO})_{3} \mathrm{~W}$ & $\mathrm{FBF}_{3}$ & 6.11 & 90 \\
& $\mathrm{OSO}_{2} \mathrm{~F}$ & 6.00 & 90 \\
& $\mathrm{OSO}_{2} \mathrm{CF}_{3}$ & $5.96^{b}$ & 90 \\
& $\mathrm{Cl}^{6}$ & 5.79 & 5
\end{tabular}

\section{A. Relative Donor Strengths of Several Weakly Coordinating Anions}

The cationic character of the metal carbonyl fragment, corresponding to a weak donor strength of the coordinated anions, can easily be derived from the $\nu$ (CO) data. The complex " $\mathrm{Cu}(\mathrm{CO}) \mathrm{AsF}_{6}$ " shows the highest $\nu(\mathrm{CO})$ frequency $\left(2180 \mathrm{~cm}^{-1}\right)$ for monocarbonyls. ${ }^{11 a}$ In the complexes $\mathrm{M}(\mathrm{CO})_{5} \mathrm{X}(\mathrm{M}=\mathrm{Mn}$, $\mathrm{Re}), \mathrm{CpM}(\mathrm{CO})_{3} \mathrm{X}(\mathrm{M}=\mathrm{Mo}, \mathrm{W})$, or $\mathrm{CpFe}(\mathrm{CO})_{2} \mathrm{X}$ the $\nu(\mathrm{CO})$ frequencies are considerably higher for $\mathrm{X}=\mathrm{EF}_{n}{ }^{-}$, $\mathrm{ClO}_{4}^{-}, \mathrm{OTeF}_{5}{ }^{-}$, etc. than in the corresponding halides. In the nitrosyl complex $\mathrm{CpW}(\mathrm{NO})_{2} \mathrm{X}$ the same observation can be made for $\nu$ (NO) (Table V).

Similar results can be obtained by comparing the ${ }^{1} \mathrm{H}$ NMR spectra of $\mathrm{CpM}(\mathrm{CO})_{3} \mathrm{X}(\mathrm{M}=\mathrm{Mo}, \mathrm{W})$, where the shifts for the cyclopentadienyl group occur at lower fields for weaker $\sigma$-donor ability of X (see Table VI). The IR and NMR data indicate the following order of 
TABLE VII

A. ${ }^{1} \mathrm{H}$ NMR Chemical Shifts and $\nu(\mathrm{Ir}-\mathrm{H})$ Wavenumbers for $\operatorname{Ir}\left(\mathrm{PPh}_{3}\right)_{2}(\mathrm{CO})(\mathrm{Cl})(\mathrm{H}) \mathrm{X}^{190}$

\begin{tabular}{lll}
\multicolumn{1}{c}{$\mathrm{X}$} & $\delta(\mathrm{Ir}-\mathrm{H})$ & $\nu(\mathrm{Ir}-\mathrm{H})$ \\
\hline $\mathrm{FBF}_{3}$ & -26.5 & 2333 \\
$\mathrm{OSO}_{2} \mathrm{CF}_{3}$ & -21.77 & 2305 \\
$\mathrm{NCSe}$ & -16.1 & \\
$\mathrm{Cl}$ & & 2240 \\
$\mathrm{SeCN}$ & -11.4 & 2180
\end{tabular}

B. ${ }^{31} \mathrm{P}$ NMR Data for trans- $\left[(\mathrm{OC})_{3}(\mathrm{ON})\left(\mathrm{Me}_{3} \mathrm{P}\right) \mathrm{WL}\right]^{+191}$

\begin{tabular}{lcc}
\hline \multicolumn{1}{c}{$\mathrm{L}$} & $\delta\left({ }^{31} \mathrm{P}\right)$ & ${ }^{1} J(\mathrm{P}-W)$ \\
\hline $\mathrm{FSbF}_{5}{ }^{-}$ & -16.25 & 282.9 \\
$\mathrm{FBF}_{3}{ }^{-}$ & -17.46 & 282.0 \\
$\mathrm{OPOF}_{2}^{-}$ & -21.48 & 274.4 \\
$\mathrm{NCMe}^{-}$ & -27.03 & 263.8 \\
$\mathrm{Cl}^{-}$ & -28.34 & 263.8 \\
$\mathrm{PPh}_{3}$ & -35.96 & 252.4
\end{tabular}

relative $\sigma$-donor strength:, $, 54,57,90 \mathrm{FAsF}_{5}^{-}, \mathrm{FSbF}_{5}{ }^{-}, \mathrm{FPF}_{5}^{-}$ $<\mathrm{FBF}_{3}^{-}<\mathrm{OSO}_{2} \mathrm{~F}^{-}, \mathrm{OSO}_{2} \mathrm{CF}_{3}^{-}<\mathrm{OClO}_{3}^{-}<\mathrm{OTeF}_{5}^{-}$, $\mathrm{OReO}_{3}^{-} \ll \mathrm{Cl}^{-}$.

A similar order was found earlier from electronic spectra and magnetic data of complexes $M(e n)_{2} X_{2}$ and $M(p y){ }_{4} X_{2} \cdot{ }^{14 c, 188} \quad$ Calculations of the "group electronegativities" 189 of the coordinating anions are consistent with this series. For complexes of the same type this trend is qualitatively also observed in the chemical reactivity. For instance, the reaction of $\mathrm{HRh}\left(\mathrm{PP}_{3}\right)\left(\mathrm{PP}_{3}=\mathrm{P}\left(\mathrm{CH}_{2} \mathrm{CH}_{2} \mathrm{PPh}_{2}\right)_{3}\right)$ with $\mathrm{HBF}_{4}$ yields an $\eta^{2}-\mathrm{H}_{2}$ complex $\left[\mathrm{H}_{2} \mathrm{Rh}\left(\mathrm{PP}_{3}\right)\right]^{+} \mathrm{BF}_{4}^{-}$, which spontaneously loses $\mathrm{H}_{2}$ on addition of $\mathrm{CF}_{3} \mathrm{SO}_{3}{ }^{-}$to give the triflato complex $\left(\mathrm{PP}_{3}\right) \mathrm{RhOSO}_{2} \mathrm{CF}_{3}$. ${ }^{76 a}$

Two correlations have been found by comparing either the $\delta(\mathrm{Ir}-\mathrm{H})$ chemical shifts and $\nu(\mathrm{Ir}-\mathrm{H})$ in compounds $\mathrm{HIr}(\mathrm{CO})\left(\mathrm{PPh}_{3}\right)_{2}(\mathrm{~L}) \mathrm{Cl}^{190}$ or the ${ }^{31} \mathrm{P}$ NMR data of $\mathrm{LW}(\mathrm{NO})\left(\mathrm{PMe}_{3}\right)(\mathrm{CO})_{3}{ }_{3}^{191}(\mathrm{~L}=$ anionic or neutral ligand). Linear relationships between the $\delta(\operatorname{Ir}-H)$ shift and the electronegativity of the ligand trans to hydrogen in the first case and between ${ }^{31} \mathrm{P}$ NMR shifts and one-bond coupling constants ${ }^{1} J\left({ }^{31} \mathrm{P}-183 \mathrm{~W}\right)$ in the second case were found. Both measurements allow one to derive a general order of $\sigma$-donor strengths for both anionic or neutral ligands: $\mathrm{F}$ donor $<\mathrm{O}$ donor $<\mathrm{N}$ donor (Table VII).

From the porphyrin core coordination parameters of five-coordinate ferric porphyrins, e.g., the iron-nitrogen distances, or the distance of iron atoms from the porphyrin plane, the following order of decreasing strength of anion bonding has been found: ${ }^{180 \mathrm{~b}} \mathrm{I}^{-}>\mathrm{OClO}_{3}{ }^{-}>$ $\mathrm{FSbF}_{5}{ }^{-}>\mathrm{B}_{11} \mathrm{CH}_{12}^{-}$. Thus the carborane ligand is the least coordinating anion, at least for the $\mathrm{Fe}(\mathrm{TPP})^{+}$ moiety. ${ }^{179,180} \mathrm{~A}$ "new" candidate for the least coordinating anion is the $\mathrm{B}\left(\mathrm{OTeF}_{5}\right)_{4}^{-}$anion. ${ }^{192} \mathrm{An}$ "old" candidate for this position may be the $\mathrm{C}\left(\mathrm{SO}_{2} \mathrm{R}\right)_{3}{ }^{-}$ion, which has been shown to be a very weak ligand. ${ }^{193}$

\section{B. Substitution of Weakly Coordlnating Llgands by Noutral $\sigma$ - and $\pi$-Donors}

The weakly coordinated anions are readily displaced under very mild conditions by a large number of neutral $\sigma$ - and $\pi$-donors to give ionic complexes

$$
\mathrm{L}_{n} \mathrm{M}-\mathrm{X}+\mathrm{L}^{\prime} \rightarrow\left[\mathrm{L}_{n} \mathrm{M}-\mathrm{L}^{\prime}\right]^{+} \mathrm{X}^{-}
$$

where $\mathrm{X}=\mathrm{BF}_{4}^{-}, \mathrm{PF}_{6}^{-}, \mathrm{AsF}_{6}^{-}, \mathrm{SbF}_{6}^{-}, \mathrm{OTeF}_{5}^{-}, \mathrm{OClO}_{3}^{-}$, $\mathrm{CF}_{3} \mathrm{SO}_{3}^{-}, \mathrm{AlCl}_{4}^{-}$, and $\mathrm{SbCl}_{6}^{-}$and $\mathrm{L}^{\prime}=\mathrm{H}_{2} \mathrm{O}$, $\mathrm{ROH}^{84 \mathrm{a}, 194,195} \mathrm{R}_{2} \mathrm{O},{ }^{58,195,196} \mathrm{R}_{2} \mathrm{C}=\mathrm{O},{ }^{5,42,49,50,55,56 \mathrm{a}, 134,194,198,199}$
$\mathrm{RNO}_{2},{ }^{134,200} \mathrm{H}_{2} \mathrm{~S}^{24,201,202} \mathrm{RSH}^{202,203} \mathrm{R}_{2} \mathrm{E}_{n}(n=1,2 ; \mathrm{E}$ $=\mathrm{S}, \mathrm{Se}, \mathrm{Te}),{ }^{103,200,202,204,205} \quad \mathrm{R}_{2} \mathrm{SO},{ }^{202,205} \quad \mathrm{~N}_{2},{ }^{74,169}$ $\mathrm{NR}_{3},{ }^{169,206-209,282} \mathrm{R}-\mathrm{CN},{ }^{134,210-213} \mathrm{ER}_{3}(\mathrm{E}=\mathrm{P}, \mathrm{As}, \mathrm{Sb}$, $\mathrm{Bi})$, ${ }^{135 e, 205,214-218} \mathrm{R}_{3} \mathrm{EX}$ ( $\mathrm{E}=\mathrm{P}, \mathrm{As}, \mathrm{Sb} ; \mathrm{X}=\mathrm{S}, \mathrm{Se}, \mathrm{Te}$, $\mathrm{O}, \mathrm{NR}){ }^{219,282} \mathrm{RN} \equiv \mathrm{C},{ }^{220,245} \mathrm{R}_{2} \mathrm{C}=\mathrm{CR}_{2},{ }^{10,134,136 a, 182,221-226}$ $\mathrm{R}-\mathrm{C} \equiv \mathrm{C}-\mathrm{R},{ }^{5,51,136 \mathrm{~b}, 225,227-229} \mathrm{CO},{ }^{5,42,62} \mathrm{SO}_{2},{ }^{194,213} \mathrm{O}, \mathrm{S}$, and $\mathrm{N}$ heterocycles, ${ }^{230,239 \mathrm{~b}}$ and guanine ${ }^{239 \mathrm{c}}$ and xanthine derivatives. ${ }^{77}$ Easy uptake of $\mathrm{H}_{2}$ even in the solid state has been reported for $\left[\mathrm{Co}\left(\mathrm{P}(\mathrm{OMe})_{3}\right)_{4}\right]^{+} \mathrm{PF}_{6}{ }^{-}$to give $\left[\mathrm{H}_{2} \mathrm{Co}\left(\mathrm{P}(\mathrm{OMe})_{3}\right)_{4}\right]^{+} \mathrm{PF}_{6}{ }^{-10}$

The systems $\mathrm{CpFe}(\mathrm{CO})_{2} \mathrm{X},{ }^{231 \mathrm{a}} \mathrm{CpML}_{2} \mathrm{X}(\mathrm{M}=\mathrm{Ru}$, $\mathrm{Os}),{ }^{231 \mathrm{~b}}$ and $\mathrm{CpM}(\mathrm{CO})_{2}(\mathrm{~L}) \mathrm{X}(\mathrm{M}=\mathrm{Mo}, \mathrm{W})$ have been intensively studied. It should be noted that the starting compounds $\mathrm{CpM}(\mathrm{CO})_{2}(\mathrm{~L}) \mathrm{X}(\mathrm{M}=\mathrm{Mo}, \mathrm{W})$ are extremely sensitive to moisture. The formation of the much less reactive red aqua complexes $\left[\mathrm{CpM}(\mathrm{CO})_{2} \mathrm{~L}\right.$ $\left.\left(\mathrm{H}_{2} \mathrm{O}\right)\right]^{+} \mathrm{X}^{-}$from the violet $\mathrm{BF}_{4}^{-}$complexes can easily be recognized. In the system $\mathrm{CpM}(\mathrm{CO})_{2}(\mathrm{~L}) \mathrm{X}$ we observed the following order of increasing ability to substitute weaker ligands: ${ }^{5,195,196,202} \mathrm{CH}_{2} \mathrm{Cl}_{2} \sim \mathrm{PF}_{6}{ }^{-} \sim$ $\mathrm{AsF}_{6}^{-} \sim \mathrm{SbF}_{6}^{-}<\mathrm{OEt}_{2}<\mathrm{BF}_{4}^{-}<\mathrm{H}_{2} \mathrm{~S}<\mathrm{THF}<\mathrm{Me}_{2} \mathrm{CO}$ $<\mathrm{H}_{2} \mathrm{O}<\mathrm{CF}_{3} \mathrm{SO}_{3}{ }^{-}<\mathrm{CO} \sim \mathrm{MeCN} \sim \mathrm{PR}_{3}$.

Other systems examined for substitution reactions include $\mathrm{CpW}(\mathrm{NO})_{2} \mathrm{BF}_{4},{ }^{222} \mathrm{CpCr}(\mathrm{NO})_{2} \mathrm{PF}_{6}{ }^{60} \mathrm{Mn}$ $(\mathrm{CO})_{5} \mathrm{OClO}_{3},{ }^{55,56 a, 217}(\mathrm{Mn}, \mathrm{Re})(\mathrm{CO})_{5} \mathrm{OSO}_{2} \mathrm{CF}_{3},{ }^{58}(\mathrm{Mn}$,$\mathrm{Re})(\mathrm{CO})_{5} \mathrm{FBF}_{3},{ }^{42,56 \mathrm{c}, 224,239 \mathrm{a}, \mathrm{b}} \mathrm{Mn}(\mathrm{CO})_{4}(\mathrm{~L}) \mathrm{BF}_{4},{ }^{24} \mathrm{Mn}$ $(\mathrm{CO})_{3}(\mathrm{~L})_{2} \mathrm{X}\left(\mathrm{X}=\mathrm{ClO}_{4}^{-}, \mathrm{PO}_{2} \mathrm{~F}_{2}^{-56 \mathrm{a}, 220}(\mathrm{Mn}, \mathrm{Re})(\mathrm{CO})_{5} \mathrm{~F}-\right.$ $\mathrm{AsF}_{5}, 194,213,234 \quad \mathrm{Cp}^{\prime} \mathrm{Os}(\mathrm{CO}){ }_{2} \mathrm{BF}_{4},{ }^{30}(\mathrm{Rh}, \mathrm{Ir})(\mathrm{CO})-$ $\left(\mathrm{PPh}_{3}\right)_{2} \mathrm{ClO}_{4},{ }^{62,210,211} \mathrm{HIr}(\mathrm{Cl})(\mathrm{CO})\left(\mathrm{PPh}_{3}\right)_{2} \mathrm{FBF}_{3},{ }^{164}$ $\mathrm{Cp}_{2} \mathrm{TiX}_{2},{ }^{198,206,212}$ and $\mathrm{W}(\mathrm{CO})_{3}\left(\mathrm{PR}_{3}\right)(\mathrm{NO}) \mathrm{X}^{191}(\mathrm{OC})_{5^{-}}$ $\mathrm{ReOSO}_{2} \mathrm{CF}_{3}$ undergoes substitution of triflate with nucleophiles according to an associative process. ${ }^{58} \mathrm{In}$ terestingly, the reactions of $\mathrm{CpRe}(\mathrm{NO})\left(\mathrm{PPh}_{3}\right) \mathrm{X}\left(\mathrm{X}^{-}=\right.$ $\mathrm{BF}_{4}^{-}, \mathrm{PF}_{6}^{-}$) with $\sigma$ - and $\pi$-donors proceed with retention of configuration at rhenium. .11 $^{\text {b }}$

Many substitution reactions have been carried out by in situ generation of $\mathrm{L}_{n} \mathrm{M}-\mathrm{X}$ or from cationic solvent complexes in the presence of donors. As far as these syntheses have been carried out in dichloromethane, coordination of $\mathrm{CH}_{2} \mathrm{Cl}_{2}$ may occur. $5,52,53,56 \mathrm{~b}, 91 \mathrm{~b}, 186 \mathrm{a}, 22 \mathrm{I}$

Interesting ligands that could be coordinated to the metal via this method in cationic complexes are acetic and trifluoroacetic acid, ${ }^{232} \mathrm{CH}_{3} \mathrm{I},{ }^{233 a, e, 236} \mathrm{C}_{6} \mathrm{H}_{4} \mathrm{I}_{2},{ }^{233 \mathrm{~b}}$ $\mathrm{NS}^{+234 \mathrm{a}}$ (thus a dicationic carbonyl complex $\left[(\mathrm{OC})_{5} \mathrm{ReNS}\right]^{2+}$ could be obtained), $\mathrm{NSF}_{3}{ }^{234 \mathrm{~b}} \mathrm{HNSO},{ }^{234 \mathrm{c}}$ $\mathrm{HNSOF}_{2}$, ${ }^{234 d} \mathrm{Ph}_{3} \mathrm{PCHCOCH}_{3}{ }^{235}$ and $\mathrm{Et}_{3} \mathrm{PCS}_{2}{ }^{237}$ The alkyl iodide complexes $\left[\operatorname{Ir}\left(\mathrm{PPh}_{3}\right)_{2} \mathrm{H}_{2}(\mathrm{MeI})_{2}\right]^{+233 \mathrm{a}}$ and $\left[\mathrm{CpRe}(\mathrm{NO})\left(\mathrm{PPh}_{3}\right)\left(\mathrm{ICH}_{2} \mathrm{SiMe}_{3}\right)\right]^{+} \mathrm{BF}_{4}^{-236}$ have been characterized by crystal structure determination. Closely related are [(COD) $\operatorname{Ir}\left(\eta^{2}-(P, B r)\right.$ $\left.\left.\left(\mathrm{Ph}_{2} \mathrm{PC}_{6} \mathrm{H}_{4} \mathrm{Br}\right)\right)\right]^{+233 c}$ and $\left[\mathrm{IrH}_{2}\left(\mathrm{PPh}_{3}\right)_{2}\left(\eta^{2}\right.\right.$-(8-fluoroquinoline))].233d

With diphosphines mono- and dinuclear cationic complexes can be obtained, e.g., [(CO) ${ }_{5} \mathrm{Re}-\mathrm{PPh}_{2}-\mathrm{X}-$ $\left.\mathrm{PPh}_{2}-\mathrm{Re}(\mathrm{CO})_{5}\right]^{2+}$, with $\mathrm{X}=\mathrm{CH}_{2}, \mathrm{NH}$, or $\mathrm{O}$, or the two tautomers $\left[(\mathrm{CO})_{5} \mathrm{Re}-\mathrm{PPh}_{2}-\mathrm{NH}-\mathrm{PPh}_{2}\right]^{+}$and $\left[(\mathrm{CO})_{5} \mathrm{Re}-\right.$ $\left.\mathrm{PPh}_{2}-\mathrm{N}-\mathrm{PPh}_{2} \mathrm{H}\right]^{+} .238$ Several polyfunctional neutral ligands, such as pyrazine, ${ }^{239 b}$ TCNE, TCNQ, ${ }^{239 a} 1,3-$ or 1,4-dithiane, 1,3,5-trithiane, ${ }^{219 b, 239 d}$ or 1,4-dioxane, ${ }^{196} \mathrm{can}$ also be metalated by more than one $\operatorname{Re}(\mathrm{CO})_{5}{ }^{+}$to give ligand-bridged cations; e.g.

$$
\mathrm{L}-\mathrm{L}+2 \operatorname{Re}(\mathrm{CO})_{5}{ }^{+} \rightarrow\left[(\mathrm{OC})_{5} \mathrm{Re}-\mathrm{L}-\mathrm{L}-\operatorname{Re}(\mathrm{CO})_{5}\right]^{2+}
$$

Trithiane and TCNQ can be trimetallated by $\mathrm{Cp}(\mathrm{OC})_{2} \mathrm{Fe}^{+219 \mathrm{~b}}$ or $\operatorname{Re}(\mathrm{CO})_{5}{ }^{+}, 239 \mathrm{a}$ respectively. 
$\mathrm{CO}$ readily displaces $\mathrm{AlCl}_{4}^{-}, \mathrm{BF}_{4}^{-}$, or $\mathrm{ReF}_{6}^{-}$in $(\mathrm{Mn}$,$\mathrm{Re})(\mathrm{CO})_{5} \mathrm{X}^{42,108,109,144}$ in a CO atmosphere, while for $\mathrm{X}$ $=\mathrm{OTeF}_{5}{ }^{-}$or $\mathrm{CF}_{3} \mathrm{SO}_{3}{ }^{-}$no reaction occurs under these conditions. ${ }^{54,58}$ On the other hand, in (OC) ${ }_{5} \mathrm{MnX}$ with $\mathrm{X}=\mathrm{OTeF}_{5}{ }^{-}$and $\mathrm{CF}_{3} \mathrm{SO}_{3}{ }^{-}$ligands $\mathrm{CO}$ is substituted by nucleophiles while the anionic ligand remains coordinated ${ }^{54,58}$ Interestingly, the metal-triflate bond of (OC) ${ }_{5} \mathrm{MOSO}_{2} \mathrm{CF}_{3}(\mathrm{M}=\mathrm{Mn}, \mathrm{Re})$ is seriously weakened in superacidic solvents $\left(\mathrm{CF}_{3} \mathrm{SO}_{3} \mathrm{H}, \mathrm{FSO}_{3} \mathrm{H}, \mathrm{SbF}_{5}\right)$. In these systems the triflate complexes react with $\mathrm{CO}$ to give $\left[\mathrm{M}(\mathrm{CO})_{6}\right]^{+} .58$

Introducing alkenes or alkynes to form cationic complexes with coordinated unsaturated hydrocarbons is particularly easy by this method. Cationic complexes are important starting materials for nucleophilic addition reactions at coordinated unsaturated hydrocarbons, carbon monoxide, isonitriles, or ketones. ${ }^{518,199,240-243}$ The use of carbonyl metalates, e.g., $\operatorname{Re}(\mathrm{CO})_{5}{ }^{-}$, instead of organic nucleophiles for the attack on the coordinated unsaturated hydrocarbon leads to a series of hydrocarbon-bridged bimetallic complexes in a directed way; ${ }^{244}$ e.g.

$$
\left[(\mathrm{OC})_{5} \operatorname{Re}\left(\mathrm{C}_{2} \mathrm{H}_{4}\right)\right]^{+}+\left[\operatorname{Re}(\mathrm{CO})_{5}\right]^{-} \rightarrow{ }_{(\mathrm{OC})_{5} \mathrm{ReCH}_{2} \mathrm{CH}_{2} \operatorname{Re}(\mathrm{CO})_{5}}
$$

\section{Reaction of Tetrafluoroborato and Hexafluorophosphato Complexes with Water}

Three different types of reaction with water have been observed.

\section{Formation of Aqua Complexes}

The anion can be substituted by $\mathrm{H}_{2} \mathrm{O}$ to give cationic aqua complexes. So $\left[\mathrm{Mn}(\mathrm{CO})_{5}\left(\mathrm{H}_{2} \mathrm{O}\right)\right]^{+},{ }^{246}\left[\operatorname{Re}(\mathrm{CO})_{5^{-}}\right.$ $\left.\left(\mathrm{H}_{2} \mathrm{O}\right)\right]^{+}, 42,56 c, 194,201\left[\mathrm{Mn}(\mathrm{CO})_{4}\left(\mathrm{PPh}_{3}\right)\left(\mathrm{H}_{2} \mathrm{O}\right)\right]^{+},{ }^{24}[(\mathrm{Mn}$,$\left.\mathrm{Re})(\mathrm{CO})_{3} \mathrm{~L}_{2}\left(\mathrm{H}_{2} \mathrm{O}\right)\right]^{+}{ }^{56 \mathrm{c}}\left[\mathrm{Cp}(\mathrm{Mo}, \mathrm{W})(\mathrm{CO})_{2}(\mathrm{~L})\left(\mathrm{H}_{2} \mathrm{O}\right)\right]^{+, 5,195}$ $\left[\mathrm{CpOs}(\mathrm{CO})_{2}\left(\mathrm{H}_{2} \mathrm{O}\right)\right]^{+},{ }^{30}\left[\mathrm{Cr}(\mathrm{CO})_{3}\left(\mathrm{PMe}_{3}\right)(\mathrm{CMe})\left(\mathrm{H}_{2} \mathrm{O}\right)\right]^{+4}$ $\left[\mathrm{CpFe}(\mathrm{CO})_{2}\left(\mathrm{H}_{2} \mathrm{O}\right)\right]^{+}, 41,53 \quad\left[\mathrm{Pt}\left(\mathrm{PPh}_{3}\right)_{2}(\mathrm{Cl})\left(\mathrm{H}_{2} \mathrm{O}\right)\right]^{+},{ }^{247}$ $\left[\mathrm{Cp}_{2} \mathrm{Ti}\left(\mathrm{H}_{2} \mathrm{O}\right)_{2}\right]^{2+}, 248\left[\mathrm{Cp}_{2} \mathrm{Zr}\left(\mathrm{H}_{2} \mathrm{O}\right)_{3}\right]^{2+}, 249$ and [W$\left.\left(\mathrm{PMe}_{3}\right)_{4}\left(\mathrm{H}_{2} \mathrm{O}\right) \mathrm{H}_{2} \mathrm{~F}\right]^{+250}$ have been obtained. The origin of the water can be moist solvent ${ }^{24}$ or traces of water in the silver salts ${ }^{56 c}$ used in synthesis.

A variable-temperature ${ }^{19} \mathrm{~F}$ NMR study of [Re$(\mathrm{CO})_{3}($ tmen $\left.)\left(\mathrm{H}_{2} \mathrm{O}\right)\right]^{+}$has been undertaken. ${ }^{56 \mathrm{C}}$ The coalescence of two singlets was interpreted as evidence for an equilibrium:

$$
\mathrm{L}_{n} \mathrm{M}-\mathrm{FBF}_{3}+\mathrm{H}_{2} \mathrm{O} \rightleftarrows\left[\mathrm{L}_{n} \mathrm{M}-\mathrm{OH}_{2}\right]^{+} . . . \mathrm{FBF}_{3}^{-}
$$

However, the characteristic pattern of coordinated $\mathrm{BF}_{4}^{-}$ has not been observed.

In complexes $\left[\mathrm{L}_{n} \mathrm{M}-\mathrm{OH}_{2}\right]^{+} \mathrm{BF}_{4}^{-} \mathrm{IR}$ spectroscopy clearly reveals hydrogen bridges between coordinated $\mathrm{H}_{2} \mathrm{O}$ and $\mathrm{BF}_{4}^{-4,5,56 c, 184,247,251}$ The characteristic pattern of the $\nu$ (BF) absorptions in H-bridged species can easily be distinguished from the pattern of coordinated $\mathrm{BF}_{4}^{-}$ and of free $\mathrm{BF}_{4}^{-}$, as was shown for $\left[\mathrm{CpMo}(\mathrm{CO})_{3}\right.$ $\left.\left(\mathrm{H}_{2} \mathrm{O}\right)\right]^{+}+{ }^{5,184}$ When more than stoichiometric amounts of water are present, hydrates are formed and the IR spectrum of free $\mathrm{BF}_{4}{ }^{-}$is observed.

$$
\begin{aligned}
\mathrm{L}_{n} \mathrm{M}-\mathrm{OH}_{2}{ }^{+} \ldots \mathrm{FBF}_{3}+ & \mathrm{H}_{2} \mathrm{O} \rightarrow \\
& {\left[\mathrm{L}_{n} \mathrm{M}\left(\mathrm{OH}_{2}\right)\left(\mathrm{OH}_{2}\right)_{n}\right]^{+} \mathrm{BF}_{4}^{-} }
\end{aligned}
$$

The crystal structure determinations of the aqua complexes $\left[\operatorname{Ir}\left(\mathrm{PPh}_{3}\right)_{2}(\mathrm{Cl}) \mathrm{H}\left(\mathrm{H}_{2} \mathrm{O}\right)\right]^{+} \mathrm{BF}_{4}{ }^{-252}$ and
$\left[\left(\mathrm{C}_{7} \mathrm{H}_{7}\right) \mathrm{Mo}(\mathrm{acac})\left(\mathrm{H}_{2} \mathrm{O}\right)\right]^{+} \mathrm{BF}_{4}^{-253}$ show a cyclic structure of two water molecules doubly hydrogen bridged to two different bridging $\mathrm{BF}_{4}^{-}$anions. $\mathrm{BF}_{4}^{-}$and $\mathrm{AsF}_{6}{ }^{-}$was shown to be hydrogen bonded to two coordinated water molecules of $\left[\operatorname{Re}(\mathrm{CO})_{3}(\mathrm{tmen})\left(\mathrm{H}_{2} \mathrm{O}\right)\right]^{+}{ }^{56 \mathrm{c}}$ Also in $\left[\mathrm{Cp}_{2} \mathrm{Zr}\left(\mathrm{H}_{2} \mathrm{O}\right)_{2}(\mathrm{OTs})\right]^{+}$hydrogen bridges between $\mathrm{H}_{2} \mathrm{O}$ and the sulfonato group have been proven. ${ }^{254}$

\section{Condensation Reactions}

In the presence of weak bases cationic aqua complexes can be deprotonated, and condensation gives dinuclear hydroxo-bridged or tris(metallaoxonium) species. With $\left[\mathrm{CpCr}(\mathrm{NO})_{2}\left(\mathrm{OH}_{2}\right)\right]^{+139}$ or $\left[\mathrm{Cp}_{2} \mathrm{Zr}\right.$ $\left.\left(\mathrm{H}_{2} \mathrm{O}\right)_{3}\right]^{2+255}$ the hydroxo-bridged $\left\{\left[\mathrm{CpCr}(\mathrm{NO})_{2}\right]_{2}(\mu\right.$ $\mathrm{OH}){ }^{+} \mathrm{BF}_{4}^{-}$and $\left[\mathrm{Cp}_{2} \mathrm{Zr}_{2}(\mu-\mathrm{OH})_{2}\left(\mathrm{H}_{2} \mathrm{O}\right)_{6}\right]^{4+}$ have been obtained. On the other hand, $\left[\mathrm{CpMo}(\mathrm{CO})_{3}\left(\mathrm{H}_{2} \mathrm{O}\right)\right]^{+}$and $\left[\mathrm{CpMo}(\mathrm{NO})_{2}\left(\mathrm{H}_{2} \mathrm{O}\right)\right]^{+}$give $\left\{\left[\mathrm{CpMo}(\mathrm{CO})_{2}\right]_{3} \mathrm{O}\right\}^{+184}$ and $\left\{[\mathrm{CpMo}(\mathrm{NO})(\mathrm{OH})]_{3} \mathrm{O}\right\}^{+} .256\left\{\left[\mathrm{CpMo}(\mathrm{CO})_{2}\right]_{3} \mathrm{O}\right\}^{+}$contains semibridging $\mathrm{CO}$ groups, while the homologous $\left\{\left[\mathrm{CpMo}(\mathrm{CO})_{2}\right]_{3} \mathrm{~S}\right\}^{+}$does not. ${ }^{257}$ Oxonium salt formation is also observed in the system $\mathrm{AuPPh}_{3} \mathrm{Cl} / \mathrm{AgBF}_{4}$, where $\left\{\left[\mathrm{Au}\left(\mathrm{PPh}_{3}\right)\right]_{3} \mathrm{O}\right\}^{+}$is the reaction product in the presence of moisture. ${ }^{258} \mathrm{Oxo}$ - and/or hydroxo-bridged oligomers are also obtained by alkaline hydrolysis of $\mathrm{Fe}$ (TPP)$\mathrm{Cl},{ }^{259}\left[\mathrm{Cp}^{\prime} \mathrm{CoX}_{2}\right]_{2},{ }^{260}$ or $\mathrm{Cp}_{2} \mathrm{ZrCl}_{2}{ }^{261}$ and have been structurally characterized.

Nucleophilic attack of hydroxide on a carbon atom of a carbonyl ligand (Hieber base reaction ${ }^{262}$ ) gives the $\mathrm{CO}_{2}$-bridged complex ${ }^{201,263}$

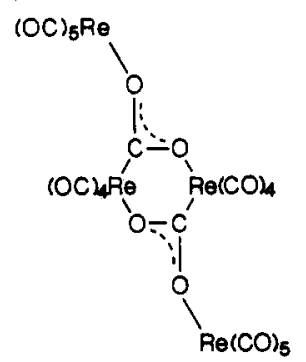

as the final product.

\section{Hydrolysis of the Anion}

Hydrolysis of the anion occurs especially with the $\mathrm{PF}_{6}{ }^{-}$anion, where the very stable $\mathrm{PO}_{2} \mathrm{~F}_{2}{ }^{-}$ion is formed, ${ }^{264}$ which is also a much stronger donor. Examples are $\mathrm{Mn}(\mathrm{CO})_{3}(\mathrm{~L})_{2} \mathrm{OPOF}_{2}{ }^{56}$ and $\mathrm{IrCl}(\mathrm{H})\left(\mathrm{PPh}_{3}\right)_{2}$ (CO) $\left(\mathrm{PO}_{2} \mathrm{~F}_{2}\right){ }^{252}$ the crystal structures of which have been determined.

In some cases hydrolysis of $\mathrm{BF}_{4}^{-}$and $\mathrm{BPh}_{4}^{-}$to give $\mathrm{BF}_{3} \mathrm{OH}^{-}$and $\mathrm{BPh}_{3} \mathrm{OH}^{-}$is observed. .2,139,201,265 $^{\text {The }}$ structure of $(\mathrm{OC})_{5} \operatorname{Re}(\mathrm{OH}) \mathrm{BF}_{3}$, which crystallized with $\left\{\left[(\mathrm{CO})_{5} \mathrm{Re}\right]_{3} \mathrm{~S}\right\}^{+} \mathrm{BF}_{4}^{-}$in the same crystal, has been determined. ${ }^{266}$ Interestingly, the $\mathrm{BF}_{3} \mathrm{OH}^{-}$ion was formed also by the reaction of $\mathrm{AgF}_{2} \mathrm{H}$ with $\mathrm{Re}(\mathrm{CO})_{3}$ (tmen) $\mathrm{Br}$ in a glass apparatus to give $\left\{\left[\mathrm{Re}(\mathrm{CO})_{3}(\mathrm{tmen}) \mathrm{F}\right]_{2} \mathrm{H}\right\}^{+}$. $\mathrm{BF}_{3} \mathrm{OH}^{-}$, as was shown by crystal structure determination. ${ }^{267}$

\section{Reaction with Monoanionic Llgands}

Particularly for $\operatorname{Re}(\mathrm{CO})_{5} \mathrm{X}$ compounds the substitution of the $\mathrm{BF}_{4}{ }^{-}$ion in $\mathrm{Re}(\mathrm{CO})_{5} \mathrm{BF}_{4}$ by a large number of anionic ligands is a straightforward way under very mild conditions. In these reactions the $\operatorname{Re}(\mathrm{CO})_{5}$ moiety remains intact, whereas in reaction of the corresponding 
halides with silver salts loss of $\mathrm{CO}$ may occur. In several cases $\mathrm{Re}(\mathrm{CO})_{5} \mathrm{FBF}_{3}$ can be reacted in aqueous solution, where substitution of the aqua ligand in $\left[\mathrm{Re}(\mathrm{CO})_{5^{-}}\right.$ $\left.\left(\mathrm{H}_{2} \mathrm{O}\right)\right]^{+}$takes place. Thus complexes $\mathrm{Re}(\mathrm{CO})_{5} \mathrm{X}$ with $\mathrm{X}=\mathrm{SCN}, \mathrm{SeCN}, \mathrm{OReO}_{3}, \mathrm{ONO}_{2}, \mathrm{NO}_{2}, \mathrm{OCHO}, \mathrm{NCNC}-$ $\mathrm{N}^{-}, \mathrm{NCC}(\mathrm{CN})_{2}{ }^{-}$, or TCNQ- could be obtained. ${ }^{57,201,239 a}$ Dicyanamide and tricyanomethanide have been shown to coordinate through the nitrile $\mathrm{N}$ atom to the metal. ${ }^{239 a}$ Related substitutions with halide, pseudohalide, or nitrate ligands have been reported for $\left[\mathrm{CpFe}(\mathrm{CO})_{2^{-}}\right.$ $\left.\left(\mathrm{OH}_{2}\right)\right]^{+} \mathrm{BF}_{4}^{-131} \mathrm{~W}(\mathrm{CO})_{4}(\mathrm{CR}) \mathrm{FBF}_{3},{ }^{120} \quad \mathrm{Cp}_{2} \mathrm{Ti}-$ $\left(\mathrm{CF}_{3} \mathrm{SO}_{3}\right)_{2},{ }^{268}$ and $\mathrm{W}(\mathrm{CO})_{2}(\mathrm{NO})\left(\mathrm{PPh}_{3}\right)_{2}\left(\mathrm{OClO}_{3}\right)^{269}$ In the perchlorato complexes $\mathrm{Mn}(\mathrm{CO})_{5}\left(\mathrm{OClO}_{3}\right)$ the $\mathrm{ClO}_{4}^{-}$ anion can be substituted by acetylide. ${ }^{270}$ Similar reactions with " $[\mathrm{CpFe}(\mathrm{CO}) \mathrm{L}]^{+» 271}$ and with $\mathrm{Os}\left(\mathrm{C}_{6} \mathrm{H}_{6}\right)(\mathrm{P}$ $i$ - $\left.\mathrm{Pr}_{3}\right) \mathrm{I}_{2} / \mathrm{AgPF}_{6}$ and an alkyne ${ }^{272}$ also yield $\sigma$-acetylide complexes $\mathrm{L}_{n} \mathrm{M}-\mathrm{C} \equiv \mathrm{C}-\mathrm{R}$. Carboxylate complexes are obtained from $\left[(\mathrm{Rh}, \mathrm{Ir})(\mathrm{CO})\left(\mathrm{PPh}_{3}\right)_{2}(\mathrm{MeCN})\right]^{+}$and peroxocarboxylates, ${ }^{273}$ from halide complexes with silver carboxylates, ${ }^{274}$ or from $\left[\left(\mathrm{Me}_{2} \mathrm{NCH}_{2}\right)_{2} \mathrm{C}_{6} \mathrm{H}_{3}(\mathrm{Ni}\right.$,$\left.\mathrm{Pt})\left(\mathrm{H}_{2} \mathrm{O}\right)\right]^{+}$and $\mathrm{NaOCHO} .^{275}$ In an interesting reaction the highly electrophilic [(OEP)Rh] ${ }^{+}$(from (OEP)RhCl and $\mathrm{AgBF}_{4}$ or $\mathrm{AgClO}_{4}$ ) yields in the presence of arenes $\mathrm{Ph}-\mathrm{X}$ the $\sigma$-aryl compounds (OEP) Rh- $\mathrm{C}_{6} \mathrm{H}_{4} \mathrm{X} .{ }^{276} \mathrm{Re}$ cently, the reaction of $\left(\mathrm{C}_{5} \mathrm{R}_{5}\right) \mathrm{M}(\mathrm{CO})_{3} \mathrm{CH}_{3} / \mathrm{HBF}_{4}(\mathrm{M}=$ $\mathrm{Mo}, \mathrm{W}$ ) with hydrogen selenide was reported to give $\left(\mathrm{C}_{5} \mathrm{R}_{5}\right) \mathrm{M}(\mathrm{CO})_{3} \mathrm{SeH}^{277}$ Metal-metal-bonded complexes can be obtained from the substitution of the weakly coordinated anion by an organometallic anion, ${ }^{278}$ e.g., $(\mathrm{OC})_{5} \mathrm{MnRe}\left({ }^{13} \mathrm{CO}\right)_{5}$ from $\left(\mathrm{O}^{13} \mathrm{C}\right)_{5} \mathrm{ReOSO}_{2} \mathrm{CF}_{3}$ and $\mathrm{KMn}(\mathrm{CO})_{5} \cdot{ }^{197}$

\section{E. Directed Synthesis of Llgand-Bridged Complexes}

\section{Neutral Ligand-Bridged Bimetallic Complexes}

It is well-known that Lewis acids of main-group elements $\left(\mathrm{BF}_{3}, \mathrm{SiF}_{4}, \mathrm{AlR}_{3}\right)$ add to the nitrogen atom of cyanide complexes. ${ }^{279}$ Similarly, the Lewis acids [Me$\left.(\mathrm{CO})\left(\mathrm{PR}_{3}\right)_{2}(\mathrm{Cl}) \mathrm{Ir}\right]^{+},{ }^{280}\left[\mathrm{Cp}_{2} \mathrm{Zr}\right]^{2+, 281}$ and $\left[\mathrm{Re}(\mathrm{CO})_{5}\right]^{+201}$ form cyanide-bridged complexes with $\left[\mathrm{Pt}(\mathrm{CN})_{4}\right]^{2-}$ or $\left[\mathrm{Au}(\mathrm{CN})_{2}\right]^{-}$, e.g., $\mathrm{NCAuCNRe}(\mathrm{CO})_{5}$ and $(\mathrm{NC})_{2} \mathrm{Pt}[\mathrm{CN}-$ $\left.\operatorname{Re}(\mathrm{CO})_{5}\right]_{2}$. By the reaction of anionic carbonyl halide or pseudohalide complexes with $\operatorname{Re}(\mathrm{CO})_{5} \mathrm{FBF}_{3}$ the neutral compounds $\mathrm{L}_{n} \mathrm{M}-\mathrm{X}-\mathrm{Re}(\mathrm{CO})_{5}$ can be obtained

$$
\begin{aligned}
{\left[\mathrm{L}_{n} \mathrm{M}-\mathrm{X}\right]^{-}+\mathrm{Re}(\mathrm{CO})_{5} } & \mathrm{FBF}_{3} \rightarrow \\
& \mathrm{L}_{n} \mathrm{M}-\mathrm{X}-\mathrm{Re}(\mathrm{CO})_{5}+\mathrm{BF}_{4}^{-}
\end{aligned}
$$

where $\mathrm{L}_{n} \mathrm{M}=\mathrm{Cr}(\mathrm{CO})_{5}$ or $\mathrm{W}(\mathrm{CO})_{5}$ and $\mathrm{X}=\mathrm{Cl}, \mathrm{Br}, \mathrm{I}$, $\mathrm{CN}, \mathrm{NCS}, \mathrm{NCO}, \mathrm{SH}$, or $\mathrm{NCC}(\mathrm{CN})_{2}{ }^{282}$ Three $\mathrm{Re}(\mathrm{CO})_{5}{ }^{+}$ groups can be added to $\left[\mathrm{Cr}(\mathrm{NCS})_{6}\right]^{3-}$ to afford $(\mathrm{SCN})_{3} \mathrm{Cr}\left[\mathrm{NCSRe}(\mathrm{CO})_{5}\right]^{3}{ }^{201}$ Examples of chalcogenide-bridged complexes obtained in a similar fashion are $\left[\left(\mathrm{C}_{5} \mathrm{R}_{5}\right)(\mathrm{CO})_{3} \mathrm{M}\right]_{2}(\mu-\mathrm{E})$, with $\mathrm{M}=\mathrm{Mo}$ or $\mathrm{W}$ and $\mathrm{S}$ or Se. ${ }^{95}$

An interesting compound, $\left[(\mathrm{OC})_{5} \mathrm{Re}-\mathrm{O}(\mathrm{O})_{2} \mathrm{CrOCr}\right.$ $\left.(\mathrm{O})_{2} \mathrm{O}-\operatorname{Re}(\mathrm{CO})_{5}\right]$, that shows that high and low oxidation states are compatible within one complex is precipitated from an aqueous solution of $\left[\mathrm{Re}(\mathrm{CO})_{5}\left(\mathrm{H}_{2} \mathrm{O}\right)\right]^{+} \mathrm{BF}_{4}^{-}$and $\mathrm{K}_{2} \mathrm{Cr}_{2} \mathrm{O}_{7} \cdot{ }^{239 \mathrm{~d}}$

From anionic acyl complexes and $\mathrm{Re}(\mathrm{CO})_{5} \mathrm{FBF}_{3}$ Fischer-type bimetallic carbene complexes can be prepared. These reactions are another example for the isolobal ${ }^{6}$ analogy between $\operatorname{Re}(\mathrm{CO})_{5}{ }^{+}$and carbenium ions.

$$
\begin{aligned}
\mathrm{L}_{n} \mathrm{M}-\mathrm{COR}^{-}+\mathrm{Re}(\mathrm{CO})_{5} \mathrm{FBF}_{3} \rightarrow & \rightarrow \\
\mathrm{L}_{n} \mathrm{M} & =\mathrm{C}(\mathrm{R}) \mathrm{O}-\mathrm{Re}(\mathrm{CO})_{5}+\mathrm{BF}_{4}^{-}
\end{aligned}
$$

where $\mathrm{L}_{n} \mathrm{M}=\mathrm{Cr}(\mathrm{CO})_{5}, \mathrm{~W}(\mathrm{CO})_{5}, \mathrm{Cr}(\mathrm{CO})_{4}\left(\mathrm{PPh}_{3}\right)$, or $\mathrm{W}(\mathrm{CO})_{4}\left(\mathrm{PPh}_{\mathrm{g}}\right)$ and $\mathrm{R}=\mathrm{Me}, \mathrm{Ph}$, or Fc. ${ }^{282,283}$

$\mathrm{CpRe}(\mathrm{CO})(\mathrm{NO}) \mathrm{COOH}$ and $\mathrm{Cp}_{2} \mathrm{Zr}(\mathrm{Cl}) \mathrm{Me}$ yield $\mathrm{Cp}$ $(\mathrm{ON})(\mathrm{OC}) \mathrm{Re}\left(\mu-\left(\eta^{1}-\mathrm{C}: \eta^{2}-\mathrm{O}, \mathrm{O}^{\prime}\right)\right) \mathrm{ZrCp}_{2} \mathrm{Cl} ; \mathrm{CpRe}(\mathrm{NO})-$ (CO) $\mathrm{CH}_{2} \mathrm{OH}$ and $\mathrm{Cp}_{2} \mathrm{Zr}(\mathrm{Cl}) \mathrm{Me}$ give $\mathrm{Cp}(\mathrm{ON})(\mathrm{OC}) \mathrm{Re}-$ $\mathrm{CH}_{2} \mathrm{O}-\mathrm{ZrCp}_{2} \mathrm{Cl}^{284}$ The anionic $\eta^{2}$-acetaldehyde complex $\left[\mathrm{CpMo}(\mathrm{CO})_{2}\left(\eta^{2}-\mathrm{MeCHO}\right)\right]^{-}$and $\mathrm{CpMo}(\mathrm{CO})_{3} \mathrm{BF}_{4}$ give $\mathrm{CpMo}(\mathrm{CO})\left(\mu-\eta^{1}, \eta^{2}-\mathrm{MeCHO}\right)(\mu-\mathrm{CO}) \mathrm{Mo}(\mathrm{CO})_{2} \mathrm{Cp}^{285}$

Closely related is the reaction of $\mathrm{Cp}(\mathrm{CO})(\mathrm{NO}) \mathrm{Mo}-$ $(\mathrm{CROLi})^{-}$with $\left[\mathrm{CpFe}(\mathrm{CO})_{2}(\mathrm{THF})\right]^{+} \mathrm{BF}_{4}^{-}$, which led to the crystallographically characterized $\mathrm{Cp}(\mathrm{NO}) \mathrm{Mo}(\mu-$ $\mathrm{CO})[\mu-\mathrm{C}(\mathrm{O}) \mathrm{R}] \mathrm{FeCp}(\mathrm{CO})^{286}$ with a $\pi$-bonded $\mu$-acyl bridge. Here, however, metal-metal bonds are involved. From iron-bonded enolates and the Lewis acid precursors $\mathrm{AuPPh}_{3} \mathrm{Cl}$ or $\mathrm{Cp}_{2} \mathrm{ZrCl}_{2}$ bimetallic complexes with either a "keto" or an "enol" bridge are obtained: $\left[\mathrm{Cp}\left(\mathrm{Ph}_{3} \mathrm{P}\right)(\mathrm{OC}) \mathrm{Fe}-\mathrm{C}\left(\mathrm{CH}_{2}\right) \mathrm{O}-\mathrm{ZrCp}_{2} \mathrm{Cl}\right]$ and $[\mathrm{Cp}-$ $\left.\left(\mathrm{Ph}_{3} \mathrm{P}\right)(\mathrm{OC}) \mathrm{Fe}-\mathrm{C}(\mathrm{O}) \mathrm{CH}_{2}-\mathrm{AuPPh}_{3}\right]^{287}$ Bimetallic $\mu$ malonyl compounds have been obtained from in situ generated $\mathrm{Cp} * \mathrm{Re}(\mathrm{NO})\left(\mathrm{PPh}_{3}\right)\left(\mathrm{COCH}_{2} \mathrm{Li}\right)$ and $\mathrm{M}(\mathrm{CO})_{5^{-}}$ $\left(\mathrm{CF}_{3} \mathrm{SO}_{3}\right)(\mathrm{M}=\mathrm{Mn}, \mathrm{Re}) .{ }^{288}$ Also oxalate, squarate, and tetrathiosquarate dianion can function as bridges between two $\operatorname{Re}(\mathrm{CO})_{5}$ or $\mathrm{CpM}(\mathrm{CO})_{3}(\mathrm{M}=\mathrm{Mo}, \mathrm{W})$ moieties. 201,289

\section{Formation of Ligand-Bridged Cationic Complexes}

Metalation of halide, pseudohalide, or chalcogenide ligands by organometallic Lewis acids has led to a large variety of dimeric metal cations:

$$
\mathrm{L}_{n} \mathrm{M}-\mathrm{X}+\mathrm{L}_{n} \mathrm{M}^{+} \rightarrow\left[\mathrm{L}_{n} \mathrm{M}-\mathrm{X}-\mathrm{ML}_{n}\right]^{+}
$$

Multifunctional compounds $\mathrm{L}_{n} \mathrm{MX}_{m}$ can be metalated more than once. Examples for these reactions include $\left\{\left[\mathrm{CpM}(\mathrm{CO})_{3}\right]_{2} \mathrm{I}\right\}^{+}(\mathrm{M}=\mathrm{Mo}, \mathrm{W}),{ }^{290}\left[\mathrm{Cp}(\mathrm{CO})_{2} \mathrm{Fe}-\mathrm{X}-\mathrm{Re}-\right.$ $\left.(\mathrm{CO})_{5}\right]^{+}\left(\mathrm{X}=\mathrm{Cl}, \mathrm{Br}, \mathrm{I}, \mathrm{N}_{3}, \mathrm{NCO}, \mathrm{NCC}(\mathrm{CN}) \mathrm{CN}, \mathrm{SR}\right.$, $\mathrm{CSSMe}, \mathrm{PPh}_{2},{ }^{282}\left[\left(\mathrm{Ph}_{3} \mathrm{PAu}\right)_{2}(\mu-\mathrm{Cl})\right]^{+}{ }^{291}\left\{\left[(\mathrm{CO})_{5} \mathrm{M}\right]_{2}(\mu-\right.$ $\mathrm{X})\}^{+}\left(\mathrm{M}=\mathrm{Mn}, \mathrm{Re} ; \mathrm{X}=\mathrm{Cl}, \mathrm{Br}, \mathrm{I}, \mathrm{NCS},{ }^{292} \mathrm{CN}, \mathrm{HCOO}\right.$, $\left.\mathrm{F}^{201} \mathrm{~N}_{3}{ }^{324}\right),\left\{\left[\mathrm{Mo}(\mathrm{CO})_{2}(\mathrm{dppe})_{2}\right]_{2}(\mu-\mathrm{F})\right\}^{+}{ }^{293}\{[\mathrm{CpFe}-$ $\left.\left.(\mathrm{CO})_{2}\right]_{2}(\mu-\mathrm{X})\right\}^{+}\left(\mathrm{X}=\mathrm{I},{ }^{20,53} \mathrm{Cl}, \mathrm{Br},{ }^{294} \mathrm{O}_{2} \mathrm{CR}^{295}\right),\{[\mathrm{CpCr}-$ $\left.\left.(\mathrm{NO})_{2}\right]_{2}(\mu-\mathrm{Cl})\right\}^{+},{ }^{20}\left[\mathrm{Pd}_{3}(\mathrm{dppe})_{3}(\mu-\mathrm{CN})_{3}\right]^{3+}, 296 \quad[\mathrm{Pd}-$ $\left.\left.\left(\mathrm{C}_{6} \mathrm{~F}_{5}\right) \mathrm{L}_{2}\right]_{2}(\mu-\mathrm{X})\right\}^{+}\left(\mathrm{X}=\mathrm{CN}, \quad \mathrm{SCN}, \mathrm{N}_{3}\right),{ }^{297}$ $\left\{\left[\left(\left(\mathrm{Me}_{2} \mathrm{NCH}_{2}\right)_{2} \mathrm{C}_{6} \mathrm{H}_{3}\right) \mathrm{Pt}\right]_{2}(\mu-\mathrm{X})\right\}^{+}(\mathrm{X}=\mathrm{Cl}, \mathrm{Br}, \mathrm{I}, \mathrm{CN}),{ }^{298}$ $\left\{\left[\mathrm{CpW}(\mathrm{CO})_{3}\right]_{2}(\mu-\mathrm{SH})\right\}^{+}, 202 \quad\left\{\left[\mathrm{Re}(\mathrm{CO})_{5}\right]_{4}\left(\mu_{4}-\mathrm{C}_{4} \mathrm{~S}_{4}\right)\right\}^{2+}, 201$ $\left\{\mathrm{Au}\left[\mathrm{CNRe}(\mathrm{CO})_{5}\right]_{2}\right\}^{+},\left\{\mathrm{Pt}\left[\mathrm{CNRe}(\mathrm{CO})_{5}\right]_{4}\right\}^{2+}, 201$ and $\{[\mathrm{Ir}-$ $\left.\left.\left(\mathrm{PPh}_{3}\right)_{2}(\mathrm{Cl})(\mathrm{H})(\mathrm{CO})\right]_{2}(\mu-\mathrm{SeCN})\right\}^{+} .{ }^{299} \mathrm{~A}$ compound [Cp$\left.\left.(\mathrm{OC})_{2} \mathrm{Fe}\right]_{2}\left(\mu_{3}-\mathrm{CS}_{2}\right) \operatorname{Re}(\mathrm{CO})_{5}\right]^{+}$, in which carbon disulfide bridges three metals, has been obtained and structurally characterized. ${ }^{282}$ It is isoelectronic with the neutral $\left[\mathrm{Cp}(\mathrm{OC})_{2} \mathrm{Fe}\right]_{2}\left(\mu_{3}-\mathrm{CS}_{2}\right)\left[\mathrm{W}(\mathrm{CO})_{5}\right]^{300}$ Tricyanomethanide coordinated to three metals via its nitrile $\mathrm{N}$ atoms has been obtained in $\left.\left\{(\mathrm{OC})_{5} \mathrm{WNCC}_{\mathrm{CNRe}}(\mathrm{CO})_{5}\right]_{2}\right\}^{+} \mathrm{BF}_{4}{ }^{-282}$ Cationic thiolate-bridged heterometallic complexes $\left[\mathrm{CpNi}(\mu-\mathrm{SR})_{2} \mathrm{MCp}_{2}\right]^{+} \mathrm{BF}_{4}{ }^{-}$can be obtained from thiolates $\mathrm{Cp}_{2} \mathrm{M}(\mathrm{SR})_{2}(\mathrm{M}=\mathrm{Ti}, \mathrm{Mo})$ and $\left[\mathrm{Cp}_{3} \mathrm{Ni}_{2}\right]^{+} .{ }^{101 \mathrm{~b}}$ Tris(metalla)sulfonium, tris(metalla)selenonium, or tris (metalla)telluronium salts $\left[\left(\mathrm{L}_{n} \mathrm{M}\right)_{3} \mathrm{E}\right]^{+}(\mathrm{E}=\mathrm{S}, \mathrm{Se}$, Te) have been obtained from chalcogenides and organometallic Lewis acids. Structurally characterized were the star-shaped cations $\left\{\left[\operatorname{Re}(\mathrm{CO})_{5}\right]_{3} \mathrm{E}\right\}^{+}(\mathrm{E}=\mathrm{S}, \mathrm{Se}$, $\mathrm{Te})^{266}$ and $\left\{\left[\mathrm{CpFe}(\mathrm{CO})_{2}\right]_{3} \mathrm{Se}\right\}^{+} .^{301}$ Unexpectedly, the compounds $\left\{\left[\mathrm{CpFe}(\mathrm{CO})_{2}\right]_{3} \mathrm{Se}\right\}^{+}$and $\left\{\left[\mathrm{Cp}(\mathrm{CO})_{2} \mathrm{Fe}\right]_{2} \mathrm{Se}-\right.$ $\left.\mathrm{Se}-\mathrm{Fe}(\mathrm{CO})_{2} \mathrm{Cp}\right\}^{+}$are formed by reaction of $[\mathrm{CpFe}-$ $\left.(\mathrm{CO})_{2}\right]_{2} \mathrm{Se}_{n}(n=1,2)$ with $\mathrm{Re}(\mathrm{CO})_{5} \mathrm{FBF}_{3}{ }^{302}$ They are 
closely related to the $\left[\mathrm{E}(\mathrm{HgX})_{3}\right]^{+303}$ and $\{[\mathrm{Au}$ $\left.\left.\left(\mathrm{PPh}_{3}\right)\right]_{3} \mathrm{E}\right\}^{+}$salts, ${ }^{304}$ which were obtained in a different way.

Di- and tricationic ligand-bridged systems with neutral bridge ligands have been mentioned before. Other examples are $\left\{\left[(\mathrm{OC})_{5} \mathrm{Re}\right]^{2}\left(\mu-(\mathrm{NS})_{2} \mathrm{NMe}\right)\right\}^{2+}, 306\{[\mathrm{CpFe}-$ (CO) $\left.\left.{ }_{2}\right]\left(\mu-\mathrm{Ph}_{2} \mathrm{E}_{2}\right)\right\}^{2+}(\mathrm{E}=\mathrm{S}, \mathrm{Se}, \mathrm{Te}),{ }^{307}$ and $\{[\mathrm{Re}-$ $\left.\left.(\mathrm{CO})_{5}\right]_{n}\left(\mu-\mathrm{L}_{\mathrm{CN}}\right)\right\}^{n+}\left(\mathrm{L}_{\mathrm{CN}}=\mathrm{TCNE}(n=2)\right.$, TCNQ $(n=$ 3)). ${ }^{239 a}$ Two interesting types of ligand bridges occur with acetylides. From $\mathrm{Re}(\mathrm{CO})_{5} \mathrm{FBF}_{3}$ and $\mathrm{SiMe}_{3} \mathrm{C} \equiv \mathrm{CH}$ or $\mathrm{CpFe}(\mathrm{CO})_{2} \mathrm{C} \equiv \mathrm{CPh}$ the $\sigma-, \pi$-bridged complexes
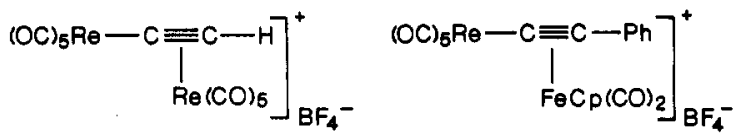

are obtained. ${ }^{282,308}$ The unexpected formation of the latter compound with a rhenium-carbon $\sigma$-bond was confirmed by X-ray crystal structure determination. ${ }^{282}$ $\mathrm{CpW}(\mathrm{CO})_{3} \mathrm{C} \equiv \mathrm{CPh}$ and $\mathrm{HBF}_{4}$ yield the structurally characterized $^{309}$

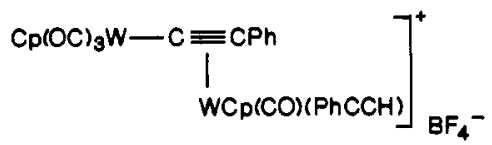

$\left[\mathrm{HPtL}_{2}\right.$ (acetone) ${ }^{+} \mathrm{BF}_{4}^{-}$and $\mathrm{PtL}_{2}(\mathrm{C} \equiv \mathrm{CPh})_{2}{ }^{310}$ or $\mathrm{Cp}$ $(\mathrm{CO})_{2} \mathrm{~W} \equiv \mathrm{CAr}^{311}$ give $\mu$-alkylidene complexes.

\section{Formation of Cationic Hydride- and Acyl-Bridged Complexes}

The concept of Lewis acid-Lewis base coupling, i.e., the reaction of organometallic Lewis acids with metal hydrides, has been proven to be a directed way for the synthesis of homo- and heterobimetallic hydridobridged complexes, which has been reviewed: ${ }^{312}$

$$
\mathrm{L}_{n} \mathrm{M}-\mathrm{H}+\mathrm{L}_{n} \mathrm{M}^{+} \rightarrow\left[\mathrm{L}_{n} \mathrm{M}-\mathrm{H}-\mathrm{ML}_{n}\right]^{+}
$$

Thus the complexes $\left[\left(\mathrm{R}_{3} \mathrm{P}\right)_{2}\left(\mathrm{R}^{\prime}\right) \mathrm{Pt}(\mu-\mathrm{H}) \mathrm{M}\left(\mathrm{PR}^{\prime \prime}{ }_{3}\right)\right]^{+}(\mathrm{M}$ $=\mathrm{Ag}, \mathrm{Au}){ }^{313}\left[\mathrm{Cp}_{2} \mathrm{M}(\mu-\mathrm{H})_{2} \mathrm{Rh}\left(\mathrm{PPh}_{3}\right)_{2}\right]^{+}(\mathrm{M}=\mathrm{Mo}, \mathrm{W}),{ }^{314}$ $\left[\mathrm{Cp}_{2} \mathrm{~W}(\mu-\mathrm{H})_{2} \mathrm{Pt}(\mathrm{Ph})\left(\mathrm{PEt}_{3}\right)\right]^{+}, 315\left[(\mathrm{CpFe})_{2}(\mu\right.$-dppe $)(\mu-$ $\mathrm{H})]^{+}, 28\left\{\left[\mathrm{CpMo}(\mathrm{CO})_{3}\right]_{2}(\mu-\mathrm{H})\right\}^{+}(\mathrm{M}=\mathrm{Mo}, \mathrm{W}),{ }^{5,316}$ and $\left\{\left[\mathrm{CpM}(\mathrm{NO})_{2}\right]_{2}(\mu-\mathrm{H})\right\}^{+}(\mathrm{M}=\mathrm{Mo}, \mathrm{W})^{317}$ have been obtained. Protonation of the dihydride $\mathrm{CpRh}\left(\mathrm{PR}_{3}\right) \mathrm{H}_{2}$ by $\mathrm{CF}_{3} \mathrm{COOH}$ yields $\left\{\left[\mathrm{CpRh}\left(\mathrm{PR}_{3}\right)\right]_{2}(\mu-\mathrm{H})_{3}\right\}^{+}{ }^{318}$ Also protonation of bimetallic neutral complexes $\left[\mathrm{CpM}(\mathrm{CO})_{3}\right]_{2}$ $(\mathrm{M}=\mathrm{Mo}, \mathrm{W}),{ }^{75}\left[\mathrm{CpFe}(\mathrm{CO})_{2}\right]_{2},{ }^{139}$ or $\mathrm{Mn}_{2}(\mathrm{CO})_{5^{-}}$ $(\mathrm{dppm})_{2}{ }^{319}$ gives hydride-bridged cationic complexes.

From transition-metal acyl complexes and organometallic Lewis acids cationic acyl-bridged bimetallic complexes can be obtained

$$
\mathrm{L}_{n} \mathrm{M}-\mathrm{C}(=\mathrm{O}) \mathrm{R}+\mathrm{L}_{n} \mathrm{M}^{+} \rightarrow\left[\mathrm{L}_{n} \mathrm{M}-\mathrm{C}(\mathrm{R})-\mathrm{O}-\mathrm{ML}_{n}\right]^{+}
$$

e.g., $\left[\mathrm{Cp}(\mathrm{CO})(\mathrm{L}) \mathrm{Fe}-\mathrm{C}\left(\mathrm{CH}_{3}\right) \mathrm{O}-\mathrm{Mo}(\mathrm{CO})_{2}(\mathrm{~L}) \mathrm{Cp}\right]^{+25,320,321}$ and $\left[\mathrm{Cp}(\mathrm{L})(\mathrm{OC})_{2} \mathrm{Mo}-\mathrm{C}\left(\mathrm{CH}_{3}\right) \mathrm{O}-\mathrm{Mo}(\mathrm{CO})_{2}(\mathrm{~L}) \mathrm{Cp}\right]^{3} .{ }^{320 b}$ These compounds can also be obtained by the reaction of transition-metal alkyl complexes with organometallic Lewis acids. It could be confirmed by a crystal structure determination ${ }^{321}$ that a symmetric acyl bridge can also be formed: ${ }^{320}$

$$
\begin{gathered}
\mathrm{CpMo}(\mathrm{CO})_{3}{ }^{+}+\mathrm{CpMo}(\mathrm{CO})_{3} \mathrm{Me} \rightarrow \\
{\left[\left[\mathrm{Cp}(\mathrm{OC})_{2} \mathrm{Mo}\right]_{2}\left(\mu-\eta^{2}-\mathrm{OCCH}_{3}\right)\right]^{+}+} \\
\left.\left[\mathrm{Cp}(\mathrm{OC})_{3} \mathrm{Mo}-\mathrm{C}\left(\mathrm{CH}_{3}\right) \mathrm{O}-\mathrm{Mo}^{+} \mathrm{CO}\right)_{3} \mathrm{Cp}\right]^{+}
\end{gathered}
$$

This reaction is another example of a Lewis acid me- diated methyl migration. ${ }^{322}$ Careful reexamination of the reaction of $\mathrm{CpMo}(\mathrm{CO})_{3} \mathrm{H}$ with ${ }^{2} \mathrm{CpMo}(\mathrm{CO})_{3}{ }^{+m 5}$ showed that actually hydrido bridges are formed and-due to the stable metal-hydrogen bondmigration of hydride to a carbonyl ligand does not occur. ${ }^{316}$

Coordination of the acyl oxygen atom in [Cp$\left.(\mathrm{OC})_{2} \mathrm{Fe}-\mathrm{CH}_{2} \mathrm{CO}-\mathrm{FeCp}(\mathrm{CO})_{2}\right]$ to ${ }^{~} \mathrm{CpFe}(\mathrm{CO})_{2}{ }^{+"}$ or " $\mathrm{Cp}_{2} \mathrm{ZrMe}$ " yields cationic trinuclear $\mu_{3}-\eta^{3}-(C, C, O)$. ketene complexes $\left[\mathrm{Cp}(\mathrm{OC})_{2} \mathrm{Fe}-\mathrm{CH}_{2} \mathrm{C}\left(\mathrm{OML}_{n}\right)-\mathrm{Fe}\right.$ $(\mathrm{CO})_{2} \mathrm{Cp}^{+}\left(\mathrm{ML}_{n}=\mathrm{CpFe}(\mathrm{CO})_{2}, \mathrm{Cp}_{2} \mathrm{ZrMe}\right){ }^{323}$

\section{F. Miscellaneous Reactlons}

\section{Stochiometric Reactions}

Organometallic Lewis acids are sufficiently electrophilic to cleave $\mathrm{M}-\mathrm{C}$ linkages in organo derivatives of the main-group elements $(\mathrm{M}=\mathrm{B}, \mathrm{Al}, \mathrm{Si}, \mathrm{Sn})$. Thus from the reaction of $\mathrm{CpW}(\mathrm{NO})_{2} \mathrm{BF}_{4}$ with $\mathrm{AlR}_{3}$ or $\mathrm{SnR}_{4}$ the compounds $\mathrm{CpW}(\mathrm{NO}){ }_{2} \mathrm{R}$ are produced. ${ }^{222} \mathrm{CpW}$ (NO) ${ }_{2} \mathrm{BF}_{4}$ and $\operatorname{Re}(\mathrm{CO})_{5} \mathrm{BF}_{4}$ react with $\mathrm{NaBPh}_{4}$ to give $\mathrm{CpW}(\mathrm{NO})_{2} \mathrm{Ph}^{222}$ and $\operatorname{Re}(\mathrm{CO})_{5} \mathrm{Ph}^{324}$ respectively. $\mathrm{CpRu}(\mathrm{L})_{2} \mathrm{X}$ and $\mathrm{NaBPh}_{4}$ yield either $\mathrm{CpRu}(\mathrm{L})_{2} \mathrm{Ph}$, $\left[\mathrm{CpRu}(\mathrm{L})_{2}\right]_{2}$, or $\left[\mathrm{CpRu}\left(\eta^{6}-P h-\mathrm{BPh}_{3}\right)\right]$, depending on the reaction conditions. ${ }^{325}$ Alkynylstannanes and -silanes and $\operatorname{Re}(\mathrm{CO})_{5} \mathrm{FBF}_{3}$ give $\sigma$-alkynyl complexes Re$(\mathrm{CO})_{5}-\mathrm{C} \equiv \mathrm{CR} .{ }^{308}$ For $\mathrm{R}=\mathrm{H}$ the $\sigma, \pi$-ethynide-bridged $\left\{\left[(\mathrm{OC})_{5} \mathrm{Re}-\mathrm{C} \equiv \mathrm{CH}\right] \mathrm{Re}(\mathrm{CO})_{5}\right\}^{+} \mathrm{BF}_{4}^{-}$is formed as the stable product, as was mentioned before. This compound can easily be deprotonated in a reversible reaction by Brönsted bases to give $(\mathrm{OC})_{5} \mathrm{Re}-\mathrm{C} \equiv \mathrm{C}-\mathrm{Re}(\mathrm{C}$ $\mathrm{O})_{5}{ }^{308}$ In a similar manner, the reaction of $\operatorname{Re}(\mathrm{CO})_{5} \mathrm{~F}$. $\mathrm{BF}_{3}$ and $\mathrm{Me}_{3} \mathrm{SiCH}=\mathrm{CH}_{2}$ produced the $\sigma, \pi$-vinylbridged complex ${ }^{238}$

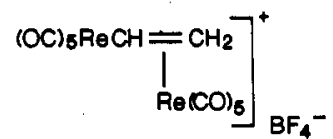

While with the organometallic Lewis acids CpMo$(\mathrm{CO})_{2}(\mathrm{~L}) \mathrm{FBF}_{3}$ and alkynes cationic $\pi$-alkyne compounds $[\mathrm{CpM}(\mathrm{CO})(\mathrm{L})(\mathrm{RCCR})]^{+} \mathrm{BF}_{4}{ }^{-5,136,227}$ are obtained, $\mathrm{M}(\mathrm{CO})_{5} \mathrm{FBF}_{3}(\mathrm{M}=\mathrm{Mn}, \mathrm{Re})$ and $\mathrm{MeCCMe}$ produce derivatives of a $(2 \pi+2 \pi)$-cycloaddition: $\mathrm{Mn}$ (CO) ${ }_{5} \mathrm{FBF}_{3}$ yields the $\pi$-cyclobutadiene compound $\mathrm{Mn}(\mathrm{CO})_{4}\left(\eta^{4}-\mathrm{C}_{4} \mathrm{Me}_{4}\right)^{+} \mathrm{BF}_{4}^{-}$with loss of carbon monoxide. ${ }^{326}$ With $\operatorname{Re}(\mathrm{CO})_{5} \mathrm{FBF}_{3}$ a $1,3-\mathrm{H}$ shift is observed and the pentacarbonyl complex

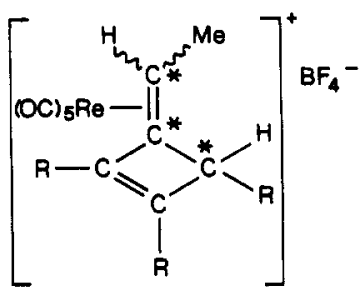

is formed as a consequence of the more stable $\operatorname{Re}(\mathrm{CO})_{5}$ unit. $^{88}$

Terminal alkynes and $\left[\mathrm{CpRu}(\mathrm{L})_{2}(\mathrm{MeOH})\right]^{+}$give rise to cationic vinylidene complexes $\left[\mathrm{Cp}\left(\mathrm{L}_{2}\right) \mathrm{Ru}=\mathrm{C}=\right.$ $\mathrm{CHR}]^{+},{ }^{228 a, b}$ while $\left[\mathrm{CpRu}(\mathrm{CO})_{2}(\mathrm{MeCN})\right]^{+} \mathrm{BF}_{4}{ }^{-}$and tolane afford the cyclobutadiene complex [CpRu( $\eta^{4}$ $\left.\left.\mathrm{C}_{4} \mathrm{Ph}_{4}\right)(\mathrm{MeCN})\right]^{+} \mathrm{BF}_{4}^{-138}$

Similar to other Lewis acids $\mathrm{CpMo}(\mathrm{CO})_{3} \mathrm{FBF}_{3}$ induces ring opening and dimerization of oxirane, and the 1,4dioxane complex $\left[\mathrm{CpMo}(\mathrm{CO})_{3} \mathrm{O}\left(\mathrm{CH}_{2}\right)_{4} \mathrm{O}\right]^{+}$is formed. ${ }^{196}$ 
$\mathrm{CpCo}(\mathrm{CO})_{2}$ reacts with $\mathrm{CpMo}(\mathrm{CO})_{3} \mathrm{FBF}_{3}{ }^{25}$ but not with $\mathrm{Re}(\mathrm{CO})_{5} \mathrm{FBF}_{3}{ }^{282}$ to yield a cationic bimetallic complex with a metal-metal bond. ${ }^{25}$ Similarly, CpM$\left(\mathrm{PMe}_{3}\right)_{2}(\mathrm{M}=\mathrm{Co}, \mathrm{Rh})$ or $\left(\mathrm{C}_{6} \mathrm{H}_{6}\right) \mathrm{Os}\left(\mathrm{PMe}_{3}\right)(\mathrm{CNR})$ and $\mathrm{AuPPh}_{3} \mathrm{Cl}$ yield $\left[\mathrm{Cp}\left(\mathrm{Me}_{3} \mathrm{P}\right)_{2} \mathrm{M}-\mathrm{AuPPh}_{3}\right]^{+} \mathrm{X}^{-327}$ and $\left[\left(\eta^{6}-\mathrm{C}_{6} \mathrm{H}_{6}\right)\left(\mathrm{Me}_{3} \mathrm{P}\right)(\mathrm{RNC}) \mathrm{Os}-\mathrm{AuPPh}_{3}\right]^{+} \mathrm{X}^{-328}$ respectively.

\section{Catalytic Reactions}

The acetone complex $\left[\mathrm{CpMo}(\mathrm{CO})_{3}\left(\mathrm{OCMe}_{2}\right)\right]^{+} \mathrm{BF}_{4}^{-}$ catalyzes the reaction of acetone with oxirane to form 1,3-dioxolane. ${ }^{196}$ E.g., alkene polymerization, oligomerization, or rearrangements can be catalyzed by $[\mathrm{Fe}$ $\left.(\mathrm{NO})_{2} \mathrm{Cl}\right]_{2} / \mathrm{AgPF}_{6},{ }^{329}\left[\mathrm{Mo}(\mathrm{NO})_{2}(\mathrm{MeCN})_{4}\right]^{2+}, 150 \mathrm{CpW}$ (NO) ${ }_{2} \mathrm{BF}_{4},{ }^{222} \mathrm{~L}_{2} \mathrm{PdCl}_{2} / \mathrm{AgBF}_{4}$ mixtures, ${ }^{330}$ or $\left(\eta^{3}\right.$ $\left.\mathrm{C}_{3} \mathrm{H}_{5}\right) \mathrm{Ni}\left(\mathrm{PMe}_{3}\right)\left(\mathrm{FBF}_{3}\right)$ and related systems $\mathrm{Ni}\left(\eta^{3}-\right.$ $\left.\mathrm{C}_{3} \mathrm{H}_{5}\right)\left(\mathrm{PR}_{3}\right)\left(\mathrm{ClAlR}_{3}\right) .{ }^{105}$ Olefin metathesis can be induced by $\mathrm{MoCl}_{2}(\mathrm{NO})_{2}\left(\mathrm{PR}_{3}\right)_{2} / \mathrm{AlEtCl}_{2}$ mixtures and similar systems, where the intermediate formation of a $\mathrm{Mo}-\mathrm{Cl}-\mathrm{AlCl}_{3}$ moiety is discussed. ${ }^{331}$ Claisen ester condensation reactions as well as the formation of 1,3 dicarbonyl compounds from 1-alkynes and acid anhydrides are catalyzed by $\mathrm{TiCl}_{2}\left(\mathrm{OSO}_{2} \mathrm{CF}_{3}\right){ }^{332}$ Diene polymerization and Diels-Alder reactions are catalyzed by $\left(\mathrm{Me}_{3} \mathrm{P}\right)(\mathrm{ON})(\mathrm{OC})_{3} \mathrm{WFSbF}_{5} ; 33$ the X-ray structure of the presumed catalytic intermediate $\left[\left(\mathrm{Me}_{3} \mathrm{P}\right)(\mathrm{ON})\right.$ $(\mathrm{OC})_{3} \mathrm{~W}$ (acrolein) $]^{+}$was determined. Hydroformylation of olefins is catalyzed by $\left[\left(\mathrm{PPh}_{3}\right)_{3} \mathrm{Rh}(\mathrm{CO})\right]^{+}[\mathrm{HC}$ $\left.\left(\mathrm{SO}_{2} \mathrm{CF}_{3}\right)_{2}\right]^{-334}$

$\operatorname{Ir}(\mathrm{CO})\left(\mathrm{PPh}_{3}\right)_{2}\left(\mathrm{OClO}_{3}\right)$ catalyzes the hydrogenation of unsaturated aldehydes. ${ }^{335}$ The most important cationic complexes are $\left[\left(\mathrm{R}_{3} \mathrm{P}\right)_{2} \mathrm{Rh}(\mathrm{solv})_{2}\right]^{+}$and $\left[\mathrm{IrH}_{2} \mathrm{~L}_{2}-\right.$ $\left.(\text { solv })_{2}\right]^{+}$for olefin hydrogenation or $\mathrm{C}-\mathrm{H}$ bond cleavage reactions, e.g., benzene formation from cyclohexane. ${ }^{336}$ This subject appears to be, however, beyond the scope of this review.

\section{Addendum}

The rapid growth of this field is documented by a series of recent interesting communications.

The complexes $\mathrm{Cp}_{2} \mathrm{Ti}\left(\mathrm{FEF}_{5}\right)_{2}(\mathrm{E}=\mathrm{As}$, Sb) have been prepared in $\mathrm{SO}_{2}$ from $\mathrm{AgEF}_{6}$ and $\mathrm{Cp}_{2} \mathrm{TiCl}_{2}{ }^{377}$ and from $\mathrm{Cp}_{2} \mathrm{TiF}_{2}$ and $\mathrm{AsF}_{5}{ }^{338}$ and the complexes $\left[\mathrm{Cp}_{2} \mathrm{Ti}-\right.$ $\left.\left(\mathrm{NCCH}_{3}\right)_{3}\right]^{2+339}$ and $\mathrm{CpTi}\left(\mathrm{SO}_{2}\right)_{n}\left(\mathrm{EF}_{6}\right)_{3}$ have been studied. ${ }^{340} \mathrm{Cp}_{2} \mathrm{Ti}\left(\mathrm{FAsF}_{5}\right)_{2}$ has been characterized by X-ray diffraction. ${ }^{338}$ The extremely reactive organometallic Lewis acid $\left[\mathrm{Cp}(\mathrm{OC})\left(\mathrm{R}_{2} \mathrm{O}\right) \mathrm{Fe}\left(\eta^{2}-\mathrm{CH}_{2}=\mathrm{CHCH}_{3}\right)\right]^{+} \mathrm{BF}_{4}{ }^{-}$ was obtained by protonation of $\mathrm{Cp}(\mathrm{OC}) \mathrm{Fe}\left(\eta^{3}\right.$-allyl $)$ with $\mathrm{HBF}_{4} \cdot \mathrm{OR}_{2}{ }^{341}$ In this paper ${ }^{341}$ previous work on the chemistry of $\mathrm{CpL}_{2} \mathrm{Fe}^{+}$cations is thoroughly described. Coordination of tetrafluoroborate could be shown by ${ }^{19} \mathrm{~F}$ NMR in $\mathrm{Cp}(\mathrm{OC})_{2} \mathrm{Fe}\left(\mathrm{FBF}_{3}\right),{ }^{341} \mathrm{Cp}(\mathrm{OC})_{2} \mathrm{Mo}\left(\eta^{2}\right.$. $\left.\mathrm{CH}_{2}=\mathrm{CHCH}_{3}\right)\left(\mathrm{FBF}_{3}\right)^{341}$ and in $\mathrm{Ph}_{3} \mathrm{P}(\mathrm{OC})_{4} \mathrm{Mn}\left(\mathrm{FBF}_{3}\right)^{342}$ Ligation of $\mathrm{CH}_{2} \mathrm{Cl}_{2}$ to the chiral Lewis acid $\mathrm{Cp}(\mathrm{ON})$ $\left(\mathrm{Ph}_{3} \mathrm{P}\right) \mathrm{Re}^{+}$has been definitely proven by low temperature ${ }^{13} \mathrm{C}$ NMR spectra. ${ }^{343}$ A hydrido-methyl complex $\left[\mathrm{Cp}(\mathrm{ON})\left(\mathrm{Ph}_{3} \mathrm{P}\right) \operatorname{Re}(\mathrm{H})(\mathrm{Me})\right]^{+}$has been detected as intermediate in the formation of $\mathrm{Cp}(\mathrm{ON})\left(\mathrm{Ph}_{3} \mathrm{P}\right) \mathrm{Re}^{+}$from $\mathrm{Cp}(\mathrm{ON})\left(\mathrm{Ph}_{3} \mathrm{P}\right) \mathrm{ReCH}_{3}$ and $\mathrm{HBF}_{4} \cdot \mathrm{OEt}_{2} \cdot{ }^{343}$ The chemistry of the stereochemically rigid Lewis acid Cp$(\mathrm{ON})\left(\mathrm{Ph}_{3} \mathrm{P}\right) \mathrm{Re}^{+}$has been extended. ${ }^{343-345}$ Impressive is the selective binding of one enantioface of monosubstituted alkenes to this cation. ${ }^{346}$ In a comprehensive study, preparation, reactions, and structures of a series of complexes $\left(\mathrm{R}_{3} \mathrm{P}\right)(\mathrm{ON})(\mathrm{OC})_{3} \mathrm{~W}\left(\mathrm{FSbF}_{5}, \mathrm{FPF}_{5}\right.$,
$\mathrm{FBF}_{3}$ ) have been described. ${ }^{347}$ NMR spectra and crystal structure determination confirmed that the anions are coordinated to tungsten both in solution and in the solid state. Apparently the strongly electron-withdrawing NO ligand - trans to the coordinated anion-increases the Lewis acidity of the metal. "Spinning" of the coordinated $\mathrm{SbF}_{6}{ }^{-}$and $\mathrm{BF}_{4}{ }^{-}$ligands in these complexes also has been observed by ${ }^{19} \mathrm{~F}$ NMR spectroscopy. Bond length comparison has been made in a series of adducts of $\mathrm{SbF}_{6}^{-}, \mathrm{BF}_{4}^{-}, \mathrm{PF}_{6}^{-}$, and $\mathrm{ReF}_{6}^{-}$. The order of binding strength in these tungsten complexes is $\mathrm{SbF}_{6}{ }^{-}$ $>\mathrm{BF}_{4}^{-}>\mathrm{PF}_{6}^{-347} \mathrm{ClO}_{4}^{-}, \mathrm{BF}_{4}^{-}$, and $\mathrm{PF}_{6}^{-}$complexes of $\mathrm{Cd}^{2+}, \mathrm{Pb}^{2+}$, and $\mathrm{Tl}^{+}$have been electrochemically generated in noncoordinating solvents and the equilibrium constants show that $\mathrm{PF}_{6}{ }_{6}$ ligation is considerably weaker than that of $\mathrm{ClO}_{4}^{-}$and $\mathrm{BF}_{4}^{-348} \mathrm{Cp}\left(\mathrm{Ph}_{3} \mathrm{P}\right)_{2} \mathrm{RuOSO}_{2} \mathrm{CF}_{3}$ reacts with thiirane to give $\left[\mathrm{Cp}\left(\mathrm{Ph}_{3} \mathrm{P}\right)_{2} \mathrm{Ru}\right.$ $\left.\left(\widetilde{\mathrm{CH}_{2} \mathrm{CH}_{2} \mathrm{~S}}\right)\right]^{+} \mathrm{SO}_{3} \mathrm{CF}_{3}{ }^{-349}$ Protonation of $\mathrm{H}_{2} \mathrm{Ru}[\mathrm{P}$ $\left(\mathrm{CH}_{2} \mathrm{CH}_{2} \mathrm{CH}_{2} \mathrm{PMe}_{2}\right)_{3} \mathrm{P}$ ] by $\mathrm{HBF}_{4}$ yields the coordinatively unsaturated complex HRu[P$\left.\left(\mathrm{CH}_{2} \mathrm{CH}_{2} \mathrm{CH}_{2} \mathrm{PMe}_{2}\right)_{3} \mathrm{P}\right]^{+} \mathrm{BF}_{4}{ }^{-350}$ The reaction of arenes with $(\mathrm{OC})_{5} \mathrm{MFBF}_{3}(\mathrm{M}=\mathrm{Mn}, \mathrm{Re})$ provides an efficient route to cationic complexes $\left[\left(\eta^{6} \text {-arene }\right) \mathrm{M}(\mathrm{CO})_{3}\right]^{+}$$\mathrm{BF}_{4}{ }^{-351,352}$

\section{Acknowledgment}

W.B. is deeply indebted to his former and present co-workers Dr. Klaus Schloter, Dr. Bernhard Olgemöller, Dr. Luitgard Olgemöller, Herbert Ernst, Dr. Karlheinz Sünkel, Dr. Günter Urban, Dr. Herbert Bauer, Dr. Klaus Raab, Dr. Mathilde Appel, Dr. Wolfgang Sacher, Dr. Jürgen Heidrich, Dr. Peter Steil, Dr. Peter M. Fritz, Dr. Martin Schweiger, Elke Fritsch, and Torsten Weidmann for their invaluable contributions to this field and Dr. Ulrich Nagel and Manfred Steimann for crystal structure determinations. We thank Hannelore Hross, Christl Fengler, and Dr. Erich Leidl for their help in preparing the manuscript and Dr. A. Kendrik for reading the manuscript. Generous support by the Deutsche Forschungsgemeinschaft and Fonds der Chemischen Industrie is gratefully acknowledged.

\section{Abbrevlations}

bpy $2,2^{\prime}$-bipyridine

COD cyclooctadiene

$\mathrm{Cp} \quad \eta^{5}$-cyclopentadienyl

Cp* $\quad \eta^{5}$-pentamethylcyclopentadienyl

dmpe bis(dimethylphosphino)ethane

dppe 1,2-bis(diphenylphosphino)ethane

en ethylene diamine

Fc ferrocenyl

nbd norbornadiene

OEP octaethylporphyrinate

OTs $p$-toluenesulfonate

py pyridine

THF tetrahydrofuran

tmen tetramethylethylenediamine

TPP tetraphenylporphyrinate

\section{References}

(1) Davies, J. A.; Hartley, F. R. Chem. Rev. 1981, 81, 79.

(2) Laing, K. R.; Robinson, S. D.; Uttley, M. F. J. Chem. Soc., Dalton Trans. 1973, 2713. 
(3) Mews, R. Angew. Chem., Int. Ed. Engl. 1975, 14, 640.

(4) Richter, K.; Fischer, E. O.; Kreiter, C. G. J. Organomet. Chem. 1976, 122, 187.

(5) Beck, W.; Schloter, K. Z. Naturforsch., B 1978, 33b, 1214. Evidence for a complex with the $\mathrm{Me} \mathrm{Si}_{2}=\mathrm{CH}_{2}$ ligand has been presented recently: Lewis, C.; Wrighton, M. S. J. Am. Chem. Soc. 1983, 105, 7768 .

(6) (a) Hoffmann, R. Angew. Chem., Int. Ed. Engl. 1982, 21, 711. (b) Stone, F. G. A. Angew. Chem., Int. Ed. Engl. 1984, 23, 89.

(7) Werner, H. Angew. Chem., Int. Ed. Engl. 1983, 22, 927. See also: Müller, H.; Vahrenkamp, H. Chem. Ber. 1980, 113, 3539 .

(8) Rosenthal, M. R. J. Chem. Educ. 1973, 50, 331 and references therein.

(9) (a) Yared, Y. W.; Miles, S. L.; Bau, R.; Reed, C. A. J. Am. Chem. Soc. 1977, 99, 7076. (b) Dedieu, A.; Hyla-Kryspin, I J. Organomet. Chem. 1981, 220,115. Siedle, A. R.; Newmark, R. A.; Pignolet, L. H.; Howells, R. D. J. Am. Chem. Soc. 1984, 106,1510 . (c) However, formation of coordinated $\mathrm{BF}_{4}^{-}$in $\mathrm{Rh}\left(\mathrm{PPh}_{3}\right)_{3} \mathrm{BF}_{4}$ has been postulated from conductivity measurements: Legzdins, P.; Mitchell, R. W.; Rempel, G. L.; Ruddick, J. D.; Wilkinson, G. J. Chem. Soc. A 1970, 3322. See also: Pimblett, G.; Garner, C. D.; Clegg, W. J. Chem. Soc., Dalton Trans. 1985, 1977.

(10) Muetterties, E. L.; Watson, P. L. J. Am. Chem. Soc. 1978 100,6978

(11) (a) Desjardins, C. D.; Edwards, D. B.; Passmore, J. Can. J. Chem. 1979, 57, 2714. (b) Doyle, G.; Eriksen, K. A.; van Engen, D. Inorg. Chem. 1983, 22, 2892.

(12) (a) Elian, M.; Hoffmann, R. Inorg. Chem. 1975, 14, 1059. (b) Hofmann, P.; Stauffert, P.; Schore, N. E. Chem. Ber. 1982, $115,2153$.

(13) (a) Driessen, R. A. J.; Hulsbergen, F. B.; Vermin, W. J.; Reedijk, J. Inorg. Chem. 1982, 21, 3594 . (b) Tomlinson, A A. G.; Bonamico, M.; Dessy, G.; Fares, V.; Scaramuzza, L. J. Chem. Soc., Dalton Trans. 1972, 1671. (c) Gaughan, A. P. Jr.; Dori, Z.; Ibers, J. A. Inorg. Chem. 1974, 13, 1657. (d) Mikulski, C. M.; Gelfand, L. S.; Schwartz, E. S. C.; Pytlewski, L. L.; Karayannis, N. M. Inorg. Chim. Acta 1980, 39, 143

(14) (a) Foley, J.; Kennefick, D.; Phelan, D.; Tyagi, S.; Hathaway, B. J. Chem. Soc., Dalton Trans. 1983, 2333. (b) Nakai, H. Bull. Chem. Soc. Jpn. 1983, 56, 1637. (c) Mayfield, H. G., Jr. Bull, W. E. J. Chem. Soc. A 1971, 2279. (d) Thomas, R. R. Chebolu, V.; Sen, A. J. Am. Chem. Soc. 1986, 108, 4096.

(15) (a) Gowda, N. M. N.; Naikar, S. B.; Reddy, G. K. N. Adv. Inorg. Chem. Radiochem. 1984, 28, 255. (b) For more recent examples, see: Fourati, M.; Chaabouni, M.; Belin, C. H. Charbonnel, M.; Pascal, J. L.; Potier, J. Inorg. Chem. 1986, 25, 1386. (c) House, D. A.; Steel, P. J.; Watson, A. A. J. Chem. Soc., Chem. Commun. 1987, 1575.

(16) Garner, C. J.; Hughes, D. Adv. Inorg. Chem. Radiochem. $1975,17,1$.

(17) Lawrance, G. A. Chem. Rev. 1986, 86, 17.

(18) Kukushkin, V. Yu.; Kukushkin, Yu. N. Sov. J. Coord. Chem. (Engl. Transl.) 1986, 11, 583

(19) (a) Jackson, W. G.; Begbie, C. M. Inorg. Chem. 1981, $20,1654$. (b) Dixon, N. E.; Jackson, W. G.; Lawrance, G. A.; Sargeson, A. M. Inorg. Synth. 1983, 22, 103. (c) Anderes, B.; Collins, S. T.; Lavaliee, D. K. Inorg. Chem. 1984, 23, 2201. (d) Harman, W. D.; Taube, H. Inorg. Chem. 1987, 26, 2917. (e) Taube, H. Pure Appl. Chem. 1979, 51, 901. (f) Harman, W. D.; Fairlie, D. P.; Taube, H. J. Am. Chem. Soc. 1986, 108, 8223.

(20) Hartmann, G.; Froböse, R.; Mews, R.; Sheldrick, G. M. $Z$. Naturforsch , $B$ 1982, 37b, 1234

(21) Deno, N. C.; Friedman, N.; Hodge, J. D.; McKay, F. P.; Saines, G. J. Am. Chem. Soc. 1962, 84, 4713.

(22) (a) Sanders, J. R. J. Chem. Soc., Dalton Trans. 1972, 1333. (b) Sanders, J. R. J. Chem. Soc., Dalton Trans. 1973, 743.

(23) Legzdins, P.; Martin, D. T. Inorg. Chem. 1979, 18, 1250.

(24) Harris, P. J.; Knox, S. A. R.; McKinney, R. J.; Stone, F. G. A. J. Chem. Soc., Dalton Trans. 1978, 1009.

(25) Sünkel, K.; Nagel, U.; Beck, W. J. Organomet. Chem. 1983, $251,227$.

(26) (a) Leoni, P.; Aquilini, E.; Pasquali, M.; Marchetti, F.; Sabat, M. J. Chem. Soc., Dalton Trans. 1988, 329. Asdar, A.; Tudoret, M.-J.; Lapinte, C. J. Organomet. Chem. 1988, 349, 343. (b) Turaki, N. N.; Huggins, J. M. Organometallics 1986, 5, 1703 .

(27) Bursten, B. E.; Gatter, M. G. J. Am. Chem. Soc. 1984, 106, 2554.

(28) LaCroce, S. J.; Menard, K. P.; Cutler, A. R. J. Organomet. Chem. 1980, 190, C79.

(29) (a) Sweet, J. R.; Graham, W. A. G. Organometallics 1983 , 2 135. (b) Sweet, J. R.; Graham, W. A. G. J. Am. Chem. Soc. $1983,105,305$.

(30) Hoyano, J. K.; May, C. J.; Graham, W. A. G. Inorg. Chem. $1982,21,3095$

(31) Sweet, J. R.; Graham, W. A. G. Organometallics 1982, 1, 982.

(32) Berke, H.; Weiler, G. Z. Naturforsch., B 1984, 39b, 431.
(33) Otto, E. E. H.; Brintzinger, H. H. J. Organomet. Chem. 1978, $148,29$.

(34) Sünkel, K. Ph.D. Thesis, Universität München, 1982. Sünkel, K.; Beck, W., unpublished.

(35) Matsui, Y.; Orchin, M. J. Organomet. Chem. 1982, 236, 381.

(36) Sanders, J. R. J. Chem. Soc., Dalton Trans. 1973, 748; 1975, 2340.

(37) Hoch, M.; Rehder, D. J. Organomet. Chem. 1985, 288, C25.

(38) Bruno, J. W.; Huffman, J. C.; Caulton, K. G. J. Am. Chem. Soc. 1984, 106, 1663

(39) Sutherland, B. R.; Cowie, M. Inorg. Chem. 1984, 23, 1290.

(40) Sweany, R. L.; Owens, J. W. J. Organomet. Chem. 1983, 255, 327.

(41) Green, M. L. H.; Nagy, P. L. I. J. Organomet. Chem. 1963, 1,58 .

(42) Raab, K.; Olgemöller, B.; Schloter, K.; Beck, W. J. Organomet. Chem. 1981, 214, 81. Raab, K.; Nagel, U.; Beck, W. Z. Naturforsch., $B$ 1983, 38b, 1466.

(43) (a) Kiel, W. A.; Lin, G.-Y.; Bodner, G. S.; Gladysz, J. A. J. Am. Chem. Soc. 1983, 105, 4958. (b) Patton, A. T.; Strouse, C. E.; Knobler, C. B.; Gladysz, J. A. J. Am. Chem. Soc. 1983, 105, 5804. (c) Asaro, M. F.; Bodner, G. S.; Gladysz, J. A.; Cooper, S. R.; Cooper, N. J. Organometallics 1985, 4, 1020. (d) Georgiou, S.; Gladysz, J. A. Tetrahedron 1986, 42, 1109 and references therein. (e) Bodner, G. S.; Gladysz, J. A.; Nielsen, M. F.; Parker, V. D. J. Am. Chem. Soc. 1987, 109, 1757.

(44) Hayes, J. C.; Jernakoff, P.; Miller, G. A.; Cooper, N. J. Pure Appl. Chem. 1984, 56, 25.

(45) E.g.: Schrock, R. R.; Sharp, P. R. J. Am. Chem. Soc. 1978, $100,2389$.

(46) Bly, R. S.; Bly, R. K.; Hossain, M. M.; Silverman, G. S.; Wallace, E. Tetrahedron 1986, 42, 1093

(47) Cutler, A. R., personal communication.

(48) Kiel, W. A.; Buhro, W. E.; Gladysz, J. A. Organometallics $1984,3,879$

(49) Appel, M.; Sacher, W.; Beck, W. J. Organomet. Chem. 1987, $322,351$.

(50) Foxman, B. M.; Klemarczyk, P. T.; Liptrot, R. E.; Rosenblum, M. J. Organomet. Chem. 1980, 187, 253 .

(51) (a) Reger, D. L.; Coleman, C. J. Organomet. Chem. 1977, 131 , 153. (b) Reger, D. L.; Coleman, C. J.; McElligott, P. J. J. Organomet. Chem. 1979, 171, 73. (c) Reger, D. L.; McElligott, P. J. J. Am. Chem. Soc. 1980, 102, 5923. (d) Reger, D. L.; Coleman, C. J. Inorg. Chem. 1979, 18, 3155. (e) Reger, D. L. Acc. Chem. Res. 1988, 21, 229.

(52) Green, M. L. H.; Whiteley, R. N. J. Chem. Soc. A 1971, 1943.

(53) Mattson, B. M.; Graham, W. A. G. Inorg. Chem. 1981, 20 3186

(54) (a) Abney, K. D.; Long, K. M.; Anderson, O. P.; Strauss, S. H. Inorg. Chem. 1987, 26, 2638. (b) Strauss, S. H.; Abney, K. D.; Long, K. M.; Anderson, O. P. Inorg. Chem. 1984, 23, 1994.

(55) Usốn, R.; Riera, V.; Gimeno, J.; Laguna, M.; Gamasa, M. P. J. Chem. Soc., Dalton Trans. 1979, 996 .

(56) (a) Wimmer, F. L.; Snow, M. R. Aust. J. Chem. 1978, 31, 267. (b) Horn, E.; Snow, M. R. Aust. J. Chem. 1980, 33, 2369. (c) Horn, E.; Snow, M. R. Aust. J. Chem. 1984, 37, 1375.

(57) Heidrich J.; Loderer, D.; Beck, W. J. Organomet. Chem. $1986,312,329$.

(58) Nitschke, J.; Schmidt, S. P.; Trogler, W. C. Inorg. Chem. 1985, 24, 1972.

(59) (a) Mallela, S. P.; Aubke, F. Inorg. Chem. 1985, 24, 2969. (b) Gerus, I. I.; Yagupol'skii, Yu. L.; Volkov, N. D. Zh. Org. Khim. 1982, 18, 1186.

(60) Regina, F. J.; Wojcicki, A. Inorg. Chem. 1980, 19, 3803.

(61) (a) Davies, J. A.; Hartley, F. R.; Murray, S. G. J. Chem. Soc., Dalton Trans. 1980, 2246. (b) Roulet, R.; Vouillamoz, R. Helv. Chim. Acta 1974, 57, 2139.

(62) Reed, C. A.; Roper, W. R. J. Chem. Soc., Dalton Trans. 1973, $1365,1370$.

(63) Colsman, M. R.; Manning, M. C.; Anderson, O. P.; Strauss, S. H. Inorg. Chem. 1987, 26,3958 .

(64) Smith, G.; Cole-Hamilton, D. J.; Gregory, A. C.; Gooden, N. G. Polyhedron 1982, 1, 97.

(65) (a) Ashworth, T. V.; Singleton, E. J. Organomet. Chem. 1974 77, C31. (b) Reimann, R. H.; Singleton, E. J. J. Organomet Chem. 1973, 59, C24.

(66) (a) Schrock, R. R.; Osborn, J. A. J. Am. Chem. Soc. 1971, 93 3089. (b) Usôn, R.; Oro, L. A.; Ciriano, M. A.; Lahoz, F. J.; Bello, M. C. J. Organomet. Chem. 1982, 234, 205.

(67) (a) Jordan, R. F.; Dasher, W. E.; Echols, S. F. J. Am. Chem Soc. 1986, 108, 1718. (b) Thewalt, U.; Lasser, W. Z. Naturforsch., $B$ 1983, 38b, 1501 .

(68) White, C.; Thompson, S. J.; Maitlis, P. M. J. Chem. Soc. Dalton Trans. 1977, 1654; 1978, 1305.

(69) Ballivet-Tkatchenko, D.; Bremard, C. J. Chem. Soc., Dalton Trans. 1983, 1143.

(70) Usôn, R.; Forniês, J.; Tomás, M.; Casas, J. M.; Cotton, F. A. Falvello, L. R. J. Am. Chem. Soc. 1985, 107, 2556. 
(71) Liston, D. J.; Reed, C. A.; Eigenbrot, C. W.; Scheidt, W. R. Inorg. Chem. 1987, 26, 2739.

(72) Sharp, P. R.; Frank, K. G. Inorg. Chem. 1985, 24, 1808.

(73) Johnson, B. F. G.; Kaner, D. A.; Lewis, J.; Raithby, P. R.; Taylor, M. J. Polyhedron 1982, 1, 105.

(74) (a) Silverthorn, W. E. J. Chem. Soc., Chem. Commun. 1971, 1310. (b) Balavoine, G.; Green, M. L. H.; Sauvage, J. P. J. Organomet. Chem. 1977, 128, 247.

(75) Davison, A.; McFarlane, W.; Pratt, L.; Wilkinson, G. J. Chem. Soc. 1962, 3653 .

(76) (a) Bianchini, C.; Mealli, C.; Peruzzini, M.; Zanobini, F. J. Am. Chem. Soc. 1987, 109, 5548. (b) Burdett, J. K.; Phillips, J. R.; Pourian, M. R.; Poliakoff, M.; Turner, J. J.; Upmacis, R. Inorg. Chem. 1987, 26, 3054. (c) Chinn, M. S.; Heinekey D. M. J. Am. Chem. Soc. 1987, 109, 5865. (d) Siedle, A. R. Newmark, R. A.; Pignolet, L. H. Inorg. Chem. 1986, 25, 3412 (e) For a recent review on $\eta^{2} \cdot \mathbf{H}_{2}$ complexes, see: Kubas, G. J. Acc. Chem. Res. 1988, 21, 120.

(77) Schweiger, M. Ph.D. Thesis, University of Munich, 1988

(78) (a) Crabtree, R. H.; Hlatky, G. G.; Holt, E. M. J. Am. Chem. Soc. 1983, 105, 7302. (b) Rhodes, L. F.; Zubkowski, J. D.; Folting, K.; Huffman, J. C.; Caulton, $\mathrm{K}$. G. Inorg. Chem. $1982,21,4185$.

(79) Ellis, R.; Henderson, R. A.; Hills, A.; Hughes, D. L. J. Organomet. Chem. 1987, 333, C6.

(80) Siedle, A. R.; Newmark, R. A.; Pignolet, L. H.; Howells, R. D. J. Am. Chem. Soc. 1984, 106, 1510

(81) (a) Allison, J. D.; Walton, R. A. J. Chem. Soc., Chem. Com mun. 1983, 401. (b) Moehring, G. A.; Walton, R. A. J. Chem. Soc., Dalton Trans. 1987, 715

(82) Werner, H.; Kletzin, H. J. Organomet. Chem. 1983, 243, C59.

(83) (a) Rhodes, L. F.; Caulton, K. G. J. Am. Chem. Soc. 1985 107, 259. (b) Crabtree, R. H.; Klatky, G. G.; Parnell, C. P. Segmüller, B. E.; Uriarte, R. J. Inorg. Chem. 1984, 23, 354 (c) Crabtree, R. H.; Hlatky, G. G. Inorg. Chem. 1984, 23 2388. (d) Harding, P. A.; Robinson, S. D. J. Chem. Soc. Dalton Trans. 1987, 947.

(84) (a) Grundy, K. R.; Robertson, K. N. Inorg. Chem. 1985, 24 3898. (b) Cameron, T. S.; Grundy, K. R.; Robertson, K. N. Inorg. Chem. 1982, 21, 4149.

(85) (a) Harding, P. A.; Preece, M.; Robinson, S. D.; Henrick, K. Inorg. Chim. Acta 1986, 118, L31. (b) Siedle, A. R.; Newmark, R. A.; Pignolet, L. H.; Wang, D. X.; Albright, T. A Organometallics 1986, 5, 38. (c) Cole-Hamilton, D. J.; Young, R. J.; Wilkinson, G. J. Chem. Soc., Dalton Trans. 1976, 1995 (d) Harding, P. A.; Robinson, S. D.; Henrick, K. J. Chem Soc., Dalton Trans. 1988, 415 .

(86) (a) Roustan, J. L. A.; Merour, J. Y.; Forgues, A. J. Organomet. Chem. 1980, 186, C23. (b) Ahmed, F. R.; Roustan, J. L. A.; Al-Janabi, M. Y. Inorg. Chem. 1985, 24, 2526.

(87) Trogler, W. C. J. Am. Chem. Soc. 1979, 101,6459

(88) Raab, K.; Beck, W. Chem. Ber. 1984, 117, 3169

(89) Griesmann, K. H.; Stasunik, A.; Angerer, W.; Malisch, W. J. Organomet. Chem. 1986, 303, C29.

(90) Appel, M.; Schloter, K.; Heidrich, J.; Beck, W. J. Organomet. Chem. 1987, 322, 77 .

(91) (a) Merrifield, J. H.; Fernändez, J. M.; Buhro, W. E.; Gladysz, J. A. Inorg. Chem, 1984, 23, 4022. (b) Fernăndez, J. M. Gladysz, J. A. Inorg. Chem. 1986, 25, 2672.

(92) Forschner, T. C.; Cutler, A. R. Organometallics 1985, 4, 1247

(93) Kletzin, H.; Werner, H. J. Organomet. Chem. 1985, 291, 213.

(94) (a) Watson, P. L.; Bergman, R. G. J. Am. Chem. Soc. 1980, 102, 2698. (b) Watson, P. L.; Bergman, R. G. J. Am. Chem. Soc. $1979,101,2055$

(95) Fischer, R. A.; Herrmann, W. A. J. Organomet. Chem. 1987, 330,377 .

(96) Flood, T. C.; Miles, D. L. J. Organomet. Chem. 1977, 127, 33.

(97) Green, M. L. H.; Massey, A. G.; Moelwyn-Hughes, J. T.; Nagy, P. L. I. J. Organomet. Chem. 1967, 8, 511 .

(98) O'Connor, E. J.; Kobayashi, M.; Floss, H. G.; Gladysz, J. A J. Am. Chem. Soc 1987, 109, 4837.

(99) (a) Anderson, S. N.; Cooksey, C. J.; Holton, S. G.; Johnson M. D. J. Am. Chem. Soc. 1980, 102, 2312. (b) Bryndza, H E.; Fong, L. K.; Paciello, R. A.; Tam, W.; Bercaw, J. E. J. Am Chem. Soc. 1987, 109, 1444

(100) Braterman, P. S. In Reactions of Coordinated Ligands; Braterman, P. S., Ed.; Plenum: New York, 1986; Vol. 1, p 37.

(101) (a) Salzer, A.; Werner, H. Angew. Chem., Int. Ed. Engl. 1972 11, 930 . (b) Werner, H.; Ulrich, B.; Schubert, U.; Hofmann P. Zimmer-Gasser, B. J Organomet. Chem, 1985, 297, 27

(102) (a) Salzer, A.; Werner, H. J. Organomet. Chem. 1975, 87, 101. (b) Salzer, A.; Werner, H. Z. Anorg. Allg. Chem. 1975, 418 88

(103) Kuhn, N.; Schumann, H.; Winter, M.; Zauder, E. Chem. Ber $1988,121,111$.

(104) (a) Markham, J.; Menard, K.; Cutler, A. Inorg. Chem. 1985 24, 1581. (b) Stuhl, L. S.; Muetterties, E. L. Inorg. Chem. 1978, 17, 2148. (c) Schrock, R. R.; Johnson, B. F.; Lewis, J. J. Chem. Soc., Dalton Trans. 1974, 951.
(105) (a) Sperling, K. Ph.D. Thesis, Universität Bochum, 1983. (b) Jolly, P. W. Comprehensive Organometallic Chemistry; Wilkinson, G., Stone, F. G. A., Abel, E. W., Eds.; Pergamon: Oxford, 1982; Vol. 8, p 615. (c) Bogdanovic, B. Adv. Organomet. Chem. 1979, 17, 105. (d) Wilke, G. Angew. Chem., Int. Ed. Engl. 1988, 27, 189.

(106) Poffenberger, C. A.; Wojcicki, A. Inorg. Chem. 1980, 19, 3795.

(107) (a) Edidin, R. T.; Norton, J. R. J. Am. Chem. Soc. 1986, 108, 948. (b) Warner, K. E.; Norton, J. R. Organometallics 1985, 4,2150 .

(108) Fischer, E. O.; Fichtel, K. Chem. Ber. 1961, 94, 1200. Fischer, E. O.; Fichtel, K.; Ofele, K. Chem. Ber. 1962, 95, 249. Fischer, E. O.; Moser, E. Inorg. Synth. 1970, 12, 35

(109) Hieber, W.; Kruck, Th. Z. Naturforsch., $B$ 1961, 16b, 709. Hieber, W.; Frey, V.; John, P. Chem. Ber. 1967, 100, 1961. Kruck, Th.; Noack, M. Chem. Ber. 1963, 96, 3028. Kruck, Th.; Höfler, M. Chem. Ber. 1963, 96, 3035.

(110) Haines, R. J.; Nyholm, R. S.; Stiddard, M. H. B. J. Chem. Soc. A 1967, 94. Treichel, P. M.; Barnett, K. W.; Shubkin, R. L. J. Organomet. Chem. 1967, 7, 449.

(111) Abel, E. W.; Tyfield, S. P. Adv. Organomet. Chem. 1970, 8, 117.

(112) Pankowski, M.; Demerseman, B.; Bouquet, G.; Bigorgne, M. J. Organomet. Chem. 1972, 35, 155 . Pankowski, M.; Bigorgne, M.; Chauvini, Y. J. Organomet. Chem. 1976, 110, 331.

(113) (a) Cotton, F. A.; Schwotzer, W. J. Am. Chem. Soc, 1986, 108 4657. (b) Cotton, F. A.; Schwotzer, W. Organometallics 1987, 6, 1275. (c) Cotton, F. A.; Schwotzer, W.; Simpson, C. Q., II. Angew. Chem., Int. Ed. Engl. 1986, 25, 637.

(114) Allegra, G.; Tettamanti Casagrande, G.; Immirzi, A.; Porri L.; Vitulli, G. J. Am. Chem. Soc. 1970, 92, 289.

(115) (a) Thewalt, U.; Stollmaier, F. J. Organomet. Chem. 1982 , 228, 149. Thewalt, U. Osterle, F. J. Organomet. Chem 1979, 172, 317. (b) Eisch, J. J.; Piotrowski, A. M.; Brownstein, S K.; Gabe, E. J.; Lee, F. L. J. Am. Chem. Soc. 1985, 107, 7219.

(116) (a) Gmelin Handbuch der Anorganischen Chemie, 8. Auflage Titan, Titanorganische Verbindungen, Teil 2, p 245; Springer-Verlag: Berlin, 1980. Appel, M. Ph.D. Thesis, Universität München, 1986. (b) Bauer, H.; Nagel, U.; Beck, W. J. Organomet. Chem. 1985, 290, 219.

(117) Scherer, O. J.; Jungmann, H. J. Organomet. Chem. 1982, 228, C61.

(118) Aylett, B. J.; Colquhoun, H. M. J. Chem. Res. (S) 1977, 148.

(119) Dodd, D.; Johnson, M. D. J. Chem. Soc. B 1971, 662.

(120) (a) Fischer, E. O.; Walz, S.; Ruhs, A.; Kreissl, F. R. Chem. Ber. 1978, 111, 2765. (b) Fischer, E. O.; Wittmann, D.; Himmelreich, D.; Schubert, U.; Ackermann, K. Chem. Ber. 1982, $115,3141$.

(121) Otto, E. E. H.; Brintzinger, H. H. J. Organomet. Chem. 1979, $170,209$.

(122) Richmond, T. G.; Basolo, F.; Shriver, D. F. Organometallics 1982, 1,1624 .

(123) Nöth, H.; Hartwimmer, R. Chem. Ber. 1960, 93, 2246.

(124) (a) Eaborn, C.; Farrell, N.; Murphy, J. L.; Pidcock, A. J. Organomet. Chem. 1973, 55, C68. (b) Eaborn, C.; Farrell, N.; Murphy, J. L.; Pidcock, A. J. Chem. Soc., Dalton Trans. 1976, 58. (c) Eaborn, C.; Farrell, N.; Pidcock, A. J. Chem. Soc., Dalton Trans. 1976, 289. (d) Connor, J. A.; Hudson, G. A. J. Organomet. Chem. 1974, 73, 351. (e) Sontag, C.; Orama, O.; Berke, H. Chem. Ber. 1987, 120,559

(125) (a) Werner, K. v.; Beck, W. Chem. Ber, 1972, 105, 3209 . (b) Beck, W.; Werner, K. v. Chem. Ber. 1971, 104, 2901. (c) Beck, W.; Kreutzer, P.; Werner, K. v. Chem. Ber. 1971, 104, 528. (d) Fehlhammer, W. P.; Beck, W.; Pöllmann, P. Chem. Ber. 1969, 102, 3903.

(126) (a) Druce, P. M.; Lappert, M. F.; Riley, P. N. K. J. Chem. Soc., Chem. Commun. 1967, 486. (b) Clark, H. C.; Dixon, K. R. Jacobs, W. J. J. Am. Chem. Soc. 1969, 91, 596, 1346.

(127) Komiya, S.; Kochi, J. K. J. Organomet. Chem. 1977, 135, 65.

(128) (a) Ishchenko, V. M.; Soloveichik, G. L.; Bulychev, B. M.; Sokolova, T. A. Russ. J. Inorg. Chem. (Engl. Transl) 1984, 29, 66. (b) Ishchenko V. M.; Bulychev, B. M.; Bel'skii, V. K. Soloveichik, G. L.; Ellert, O. G.; Seifulina, Z. M. Sov. $J$. Coord. Chem. (Engl. Transl.) 1985, 11, 483.

(129) (a) Connelly, N. G. Dahl, L. F. J. Chem. Soc., Chem. Commun. 1970, 880. (b) Reimann, R. H.; Singleton, E. J. Organomet. Chem. 1971, 32, C44; J. Chem. Soc., Dalton Trans. 1973, 2658. (c) Connelly, N. G.; Davies, J. D. J. Organomet. Chem. 1972, 38, 385. (d) Drew, D.; Darensbourg, D. J.; Darensbourg, M. Y. Inorg. Chem. 1975, 14, 1579. (e) Krusic, P. J.; Stoklosa, H.; Manzer, L. E.; Meakin, P. J. Am. Chem. Soc. 1975, 97, 667. (f) Schmidt, S. P.; Basolo, F.; Trogler, W. C. Inorg. Chim. Acta 1987, 131, 181 .

(130) Johnson, E. C.; Meyer, T. J.; Winterton, N. Inorg. Chem. $1971,10,1673$

(131) Dombek, B. D.; Angelici, R. J. Inorg. Chim. Acta 1973, 7, 345.

(132) (a) Williams, W. E.; Lalor, F. J. J. Chem. Soc., Dalton Trans. 1973, 1329. (b) Callan, B.; Manning, A. R.; Stephens, F. S. J. Organomet. Chem. 1987, 331, 357 . 
(133) Boyle, P. F.; Nicholas, K. M. J. Organomet. Chem. 1976, 114, 307.

(134) Schmidt, E. K. G.; Thiel, C. H. J. Organomet. Chem. 1981, $209,373$.

(135) (a) Catheline, D.; Astruc, D. J. Organomet. Chem. 1984, 266 C11. (b) Catheline, D.; Astruc, D. Organometallics 1984, 3, 1094. (c) Schumann, H. J. Organomet. Chem. 1986, 304, 341 (d) Schumann, H. J. Organomet. Chem. 1985, 290, C34. (e) Schumann, H. J. Organomet. Chem. 1987, 323, 193. Schumann, H. J. Organomet. Chem. 1987, 320, 145.

(136) (a) Barnes, S. G.; Green, M. J. Chem. Soc., Chem. Commun. 1980, 267. (b) Green, M. Polyhedron 1986, 5, 427.

(137) Herberhold, M.; Ott, J.; Haumaier, L. Chem. Ber. 1985, 118 3143.

(138) (a) Crocker, M.; Green, M.; Orpen, A. G.; Thomas, D. M. J Chem. Soc. Chem. Commun. 1984, 1141. (b) Crocker, M. Froom, S. F. T.; Green, M.; Nagle, K. R.; Orpen, A. G.; Thomas, D. M. J. Chem. Soc., Dalton Trans. 1987, 2803.

(139) Legzdins, P.; Martin, D. T.; Nurse, C. R.; Wassink, B. Organometallics $1983,2,1238$.

(140) Anderson, D. M.; Bristow, G. S.; Hitchcock, P. B.; Jasim, H. A.; Lappert, M. F.; Skelton, B. W. J. Chem. Soc., Dalton Trans. 1987, 2843.

(141) Herrmann, W. A. Kalcher, W. Chem. Ber. 1985, 118, 3861.

(142) Bruno, G.; Lo Schiavo, S.; Piraino, P.; Faraone, F. Organometallics 1985, 4, 1098 .

(143) Hartmann, G.; Mews, R. Chem. Ber. 1986, 119, 374.

(144) (a) Bruce, D. M.; Holloway, J. H.; Russell, D. R. J. Chem. Soc., Dalton Trans. 1978, 64. (b) Holloway, J. H.; Senior, J. B.; Szary, A. C. J. Chem. Soc., Dalton Trans. 1987, 741.

(145) Bodnar, T.; LaCroce, S. J.; Cutler, A. R. J. Am. Chem. Soc $1980,102,3292$.

(146) Ishchenko, V. M.; Bulychev, B. M.; Soloveichik, G. L.; Bel'sky, V. K.; Ellert, O. G. Polyhedron 1984, 3, 771.

(147) Yamazaki, S. Inorg. Chem. 1982, 21, 1638.

(148) McKinney, R. J. Inorg. Chem. 1982, 21, 2051.

(149) Kuhn, N.; Brüggemann, H.; Winter, M.; De Bellis, V. M. J. Organomet. Chem. 1987, 320, 391.

(150) Sen, A.; Thomas, R. R. Organometallics 1982, 1, 1251.

(151) Connor, J. A.; James, E. J.; Overton, C.; Murr, N. E. J. Chem. Soc., Dalton Trans. 1984, 255.

(152) Keller, A. Inorg. Chim. Acta 1987, 133, 207.

(153) Hunter, A. D.; Legzdins, P. Inorg. Chem. 1984, 23, 4198.

(154) Connor, J. A.; Riley, P. I. J. Chem. Soc., Dalton Trans. 1979, 1231.

(155) Treichel, P. M.; Molzahn, D. C.; Wagner, K. P. J. Organomet. Chem. 1979, 174, 191

(156) Kadish, K. M.; Lacombe, D. A.; Anderson, J. E. Inorg. Chem. $1986,25,2246$.

(157) Lacombe, D. A.; Anderson, J. E.; Kadish, K. M. Inorg. Chem. $1986,25,2074$.

(158) Ferguson, J. A.; Meyer, T. J. Inorg. Chem. 1971, 10, 1025

(159) Zecchin, S.; Zotti, G.; Pilloni, G. J. Organomet. Chem. 1985 294,379 .

(160) Connelly, N. G.; Lucy, A. R.; Payne, J. D.; Galas, A. M. R.; Geiger, W. E. J. Chem. Soc., Dalton Trans. 1983, 1879

(161) Legzdins, P. Wassink, B. Organometallics 1984, 3, 1811

(162) Hersh, W. H. J. Am. Chem. Soc. 1985, 107, 4599.

(163) Singer, H.; Wilkinson, G. J. Chem. Soc. A 1968, 2516. Strope, D.; Shriver, D. F. Inorg. Chem. 1974, 13, 2652. Burgess, J. Hacker, M. J.; Kemmitt, R. D. W. J. Organomet. Chem. 1974, 72, 121. Strope, D.; Shriver, D. F. J. Am. Chem. Soc. 1973 95, 8197. Garzon, G. Ecletica Quim. 1979, 29. Kubota, M.; McClesky, T. M.; Hayashi, R. K.; Webb, C. G. J. Am. Chem. Soc. 1987, 109, 7569 .

(164) (a) Olgemöller, B.; Bauer, H.; Beck, W. J. Organomet. Chem. 1981, 213, C57. (b) Olgemöller, B.; Bauer, H.; Löbermann, H.; Nagel, U.; Beck, W. Chem. Ber. 1982, 115, 2271. (c) Bauer, H.; Beck, W. J. Organomet. Chem. 1986, 308, 73 and references cited therein.

(165) Blake, D. M. J. Chem. Soc., Chem. Commun. 1974, 815. Smith, L. R.; Blake, D. M. J. Am. Chem. Soc. 1977, 99, 3302

(166) Olgemöller, L.; Beck, W., unpublished results.

(167) (a) Siedle, A. R.; Newmark, R. A.; Gleason, W. B. J. Am. Chem. Soc. 1986, 108, 767. (b) Siedle, A. R.; Gleason, W. B Newmark, R. A.; Pignolet, L. H. Organometallics 1986, 5 1969.

(168) Stiegman, A. E.; Tyler, D. R. J. Am. Chem. Soc. 1985, 107, 967. Philbin, C. E.; Goldmann, A. S.; Tyler, D. R. Inorg. Chem. 1986, 25, 4434.

(169) Sellmann, D.; Kleinschmidt, E. J. Organomet. Chem. 1977, 140,211

(170) Hubbard, J. L.; McVicar, W. K. J. Am. Chem. Soc. 1986, 108, 6422.

(171) (a) Jolly, P. W.; Pettit, J. J. Am. Chem. Soc. 1966, 88, 5044. (b) Green, M. L. H.; Ishaq, M.; Whiteley, R. N. J. Chem. Soc A 1967, 1508. (c) Brookhart, M.; Nelson, G. O. J. Am. Chem. Soc. 1977, 99, 6099. (d) Humphrey, M. B.; Lamanna, W. M Brookhart, M.; Husk, G. R. Inorg. Chem. 1983, 22, 3355. (e) Guerchais, V.; Astruc, D. J. Chem. Soc., Chem. Commun.
1985, 835. (f) Grötsch, G.; Boese, R.; Malisch, W. Chem. Ber. 1986, 119, 2367

(172) (a) Kegley, S. E.; Brookhart, M.;Husk, G. R. Organometallics 1982, 1,760. (b) Bodnar, T. W.; Cutler, A. R. Organometallics $1985,4,1558$.

(173) (a) Merrifield, J. H. Lin, G. Y. Kiel W. A. Gladysz, J. A. J. Am. Chem. Soc. 1983, 105, 5811. (b) Tilset, M.; Bodner, G. S.; Senn, D. R.; Gladysz, J. A.; Parker, V. D. J. Am. Chem. Soc. 1987, 109, 7551.

(174) Schneider, M.; Weiss, E. J. Organomet. Chem. 1976, 121, 365

(175) Osborne, J. H.; Rheingold, A. L.; Trogler, W. C. J. Am. Chem. Soc. 1985, 107, 6292 .

(176) Hitchcock, P. B.; Lappert, M. F.; Taylor, R. G. J. Chem. Soc., Chem. Commun. 1984, 1082.

(177) Rheingold, A. L.; Wu, G.; Heck, R. F. Inorg. Chim. Acta 1987 , $131,147$.

(178) Yamamoto, Y.; Aoki, K.; Yamazaki, H. Inorg. Chim. Acta $1982,68,75$

(179) Shelly, K.; Bartczak, T.; Scheidt, W. R.; Reed, C. A. Inorg. Chem. 1985, 24, 4325.

(180) (a) Gupta, G. P.; Lang, G.; Lee, Y. J.; Scheidt, W. R.; Shelly K.; Reed, C. A. Inorg. Chem. 1987, 26, 3022. (b) Shelly, K.; Reed, C. A.; Lee, Y. J.; Scheidt, W. R. J. Am. Chem. Soc. 1986, 108, 3117. (c) Shelly, K.; Finster, D. C.; Lee, Y. J. Scheidt, W. R.; Reed, C. A. J. Am. Chem. Soc. 1985, 107 5955 .

(181) Bell, S. A.; Lancaster, J. C.; McWhinnie, W. R. Inorg. Nucl. Chem. Lett. 1971, 7, 405.

(182) Sünkel, K.; Ernst, H.; Beck, W. Z. Naturforsch., B 1981, 36b, 474.

(183) Raab, K.; Nagel, U.; Beck, W. Z. Naturforsch., B 1983, 38b, 1466.

(184) Schloter, K.; Nagel, U.; Beck, W. Chem. Ber. 1980, 113, 3775.

(185) Legzdins, P.; Oxley, J. C. Inorg. Chem. 1984, 23, 1053.

(186) (a) Sünkel, K.; Urban, G.; Beck, W. J. Organomet. Chem. 1983, 252, 187. (b) Appel, M.; Beck, W. J. Organomet. Chem. $1987,319, \mathrm{C} 1$.

(187) Isobe, K.; Nanjo, K.; Nakamura, Y.; Kawaguchi, S. Chem. Lett. 1979, 1193

(188) (a) Morrison, R. M.; Thompson, R. C. Can. J. Chem. 1982 60, 1048. (b) Rowley, D. A.; Drago, R. S. Inorg. Chem. 1968, 7 795. (c) Byington, A. R.; Bull, W. E Inorg. Chim. Acto 1977, 21, 239. (d) Hathaway, B. J.; Billing, D. E.; Nicholls, P.; Procter, I. M. J. Chem. Soc. A 1969, 319.

(189) (a) Bratsch, S. G. J. Chem. Educ. 1985, 62, 101. (b) Mullay, J. J. Am. Chem. Soc. 1985, 107, 7271.

(190) Olgemöller, B.; Beck, W. Inorg. Chem. 1983, 22, 997.

(191) Honeychuck, R. V.; Hersh, W. H. Inorg. Chem. 1987, 26 , 1826.

(192) Noirot, M. D.; Anderson, O. P.; Strauss, S. H. Inorg. Chem. $1987,26,2216$.

(193) Beck, W.; Johansen, O.; Fehlhammer, W. P. Z. Anorg. Allg. Chem. 1968, 361, 147.

(194) Oltmanns, M.; Mews, R. Z. Naturforsch., B 1980, 35b, 1324.

(195) Sünkel, K.; Urban, G.; Beck, W. J. Organomet. Chem. 1985, $290,231$.

(196) Schloter, K. Beck, W, Z. Naturforsch , B 1980, 35b, 985

(197) Schmidt, S. P.; Basolo, F.; Jensen, C. M.; Trogler, W. C. J. Am. Chem. Soc. 1986, 108, 1894.

(198) Honold, B.; Thewalt, U. J. Organomet. Chem. 1986, 316, 291.

(199) Fernändez, J. M.; Emerson, K.; Larsen, R. D. Gladysz, J. A. J. Am. Chem. Soc. 1986, 108, 8268; J. Chem. Soc., Chem. Commun. 1988, 37.

(200) Crabtree, R. H.; Davis, M. W.; Mellea, M. F; Mihelcic, J. M. Inorg. Chim. Acta 1983, 72, 223 .

(201) Raab, K.; Beck, W. Chem. Ber. 1985, 118, 3830.

(202) Urban, G.; Sünkel, K.; Beck, W. J. Organomet. Chem. 1985, $290,329$.

(203) Treichel, P. M.; Rosenhein, L. D. Inorg. Chem. 1981, 20,942

(204) Kuhn, N.; Schumann, H. J. Organomet. Chem. 1984, 276, 55. Schumann, H. Chem.-Ztg. 1987, 111, 65. Kuhn, N.; Schumann, H.; Zauder, E. J. Organomet. Chem. 1987, 327, 17. Kuhn, N.; Schumann, H. J. Organomet. Chem. 1985, 287, 345.

(205) Usỏn, R.; Oro, L. A.; Carmona, D.; Esteruelas, M. A. J. Or. ganomet. Chem. 1984, 263, 109 .

(206) Thewalt, U.; Berhalter, K. J. Organomet. Chem. 1986, 302 , 193.

(207) Angelici, R. J; Dunker, J. W. Inorg. Chem. 1985, 24, 2209

(208) Grove, D. M.; Van Koten, G.; Ubbels, H. J. C.; Vrieze, K.; Niemann, L. C.; Stam, C. H. J. Chem. Soc., Dalton Trans. 1986, 717 .

(209) Usōn, R.; Oro, L. A.; Carmona, D.; Esteruelas, M. A. Inorg. Chim. Acta 1983, 73, 275

(210) Lee, M. K.; Kim, I. B.; Chin, C. S. J. Organomet. Chem. 1985, 290,115 .

(211) Park, S. H.; Park, H.-K.; Chin, C. S. Inorg. Chem. 1985, 24, 1120.

(212) Berhalter, K.; Thewalt, U. J. Organomet. Chem. 1987, 332 , 123. 
(213) Froböse, R.; Mews, R.; Glemser, O. Z. Naturforsch., B 1979 , $34 b, 1461$.

(214) Laguna, A.; Laguna, M.; Fananas, J.; Jones, P. G.; Fittschen, C. Inorg. Chim. Acta 1986, 121, 39.

(215) Dey, K.; Koner, D.; Ray, S. J. Chem. Soc., Dalton Trans. $1982,2385$.

(216) Hammud, H. H.; Moran, G. M. J. Organomet. Chem. 1986 , $307,255$.

(217) Carriedo, G. A.; Riera, V.; Rodriguez, M. L.; Sainz-Velicia, J J. Polyhedron $1987,6,1879$

(218) (a) Schumann, H. Chem.-Ztg. 1986, 110, 121, 161. (b) Schumann, H. J. Organomet. Chem. 1986, 299, 169. (c) Schumann, H. J. Organomet. Chem. 1985, 293, 75.

(219) (a) Kuhn, N.; Schumann, H. J. Organomet. Chem. 1986, 304 181. (b) J. Organomet. Chem. 1986, 315, 93. (c) Kuhn, N.; Schumann, H. J. Chem. Soc., Dalton Trans. 1987, 541

(220) Garcia Alonso, F. J.; Riera, V.; Villafane, F.; Vivanco, M. J. Organomet. Chem. 1984, 276, 39.

(221) Schmidt, E. K. G.; Thiel, C. H. J. Organomet. Chem. 1981 220,87 and references therein.

(222) Legzdins, P.; Martin, D. T. Organometallics 1983, 2, 1785.

(223) Olgemöller, B.; Beck, W. Angew. Chem., Int. Ed. Engl. 1980, $19,834$.

(224) Beck, W.; Raab, K.; Nagel, U.; Sacher, W. Angew. Chem., Int. Ed. Engl. 1985, 24, 505

(225) (a) Bottrill, M.; Green, M. J. Chem. Soc., Dalton Trans. 1977 2365. (b) Allen, S. R.; Baker, P. K.; Barnes, S. G.; Green, M.; Trollope, L.; Manojlovic-Muir, L.; Muir, K. W. J. Chem. Soc. Dalton Trans. 1981, 873. Green, M. J. Organomet. Chem. 1986, 300,93 and literature cited therein.

(226) Hughes, R. P.; Reisch, J. W.; Rheingold, A. L. Organometallics $1985,4,241$.

(227) Sünkel, K.; Nagel, U.; Beck, W. J. Organomet. Chem. 1981, 222,251

(228) (a) Bruce, M. I.; Wallis, R. C. Aust. J. Chem. 1979, 32, 1471. (b) Bruce, M. I.; Wong, F. S. J. Organomet. Chem. 1981, 210 C5. (c) Treichel, P. M.; Komar, D. A. Inorg. Chim. Acta 1980, $42,277$.

(229) Ashley-Smith, J.; Johnson, B. F. G.; Segal, J. A. J. Organomet. Chem. 1973, 49, C38.

(230) Sauer, N. N.; Angelici, R. J. Inorg. Chem. 1987, 26, 2160.

(231) (a) Rosenblum, M. Acc. Chem. Res. 1974, 7, 122; J. Organomet. Chem 1985, 300, 191. (b) For a recent review see Albers, M. O.; Robinson, D. J.; Singleton, E. Coord. Chem Rev. 1987, 79, 1. Consiglio, G.; Morandini, F. Chem. Rev. $1987,87,761$

(232) (a) Alt, H. G.; Hayen, H. I. J. Organomet. Chem. 1986, 316 301. (b) Werner, H.; Roll, J.; Zolk, R.; Thometzek, P.; Linse, K.; Ziegler, M. L. Chem. Ber. 1987, $120,1553$.

(233) (a) Burk, M. J.; Segmuller, B.; Crabtree, R. H. Organometallics 1987, 6, 2241. (b) Crabtree, R. H.; Faller, J. W. Mellea, M. F.; Quirk, J. M. Organometallics 1982, 1, 1361. (c) Burk, M. J.; Crabtree, R. H.; Holt, E. M. Organometallics 1984, 3, 638. (d) Kulawiec, R. J.; Holt, E. M.; Lavin, M. Crabtree, R. H. Inorg. Chem. 1987, 26, 2559. (e) Kulawiec, R. J.; Crabtree, R. H. Organometallics 1988, 7, 1891.

(234) (a) Hartmann, G.; Mews, R. Angew. Chem., Int. Ed. Engl. 1985, 24, 202. (b) Mews, R.; Glemser, O. Angew. Chem. Int Ed. Engl. 1975, 14, 186. (c) Hartmann, G.; Hoppenheit, R. Mews, R. Inorg. Chim. Acta 1983, 76, L201. (d) Mews, R. Braeuer, H. C. Z. Anorg. Allg. Chem. 1978, 447, 126.

(235) Usoon, R.; Forniês, J.; Navarro, R.; Espinet, P.; Mendivil, C. J. Organomet. Chem. 1985, 290, 125.

(236) Winter, C. H.; Arif, A. M.; Gladysz, J. A. J. Am. Chem. Soc. $1987,109,7560$

(237) Usón, R.; Fornies, J.; Navarro, R.; Usón, M. A.; Garcia, M. P.; Welch, A. J. J. Chem. Soc., Dalton Trans. 1984, 345 .

(238) Steil, P.; Beck, W., unpublished results.

(239) (a) Sacher, W.; Nagel, U.; Beck, W. Chem. Ber. 1987, 120 895. (b) Appel, M.; Sacher, W.; Beck, W. J. Organomet. Chem. 1987, 333, 237. (c) Bauer, H.; Sheldrick, G. M.; Nagel U.; Beck, W. $Z$. Naturforsch., $B$ 1985, 40b, 1237. (d) Heidrich, J.; Beck, W. J. Organomet. Chem. 1988, 354, 91.

(240) Collman, J. P.; Hegedus, L. S.; Norton, J. R.; Finke, R. G Principles and Applications of Organotransition Metals; University Science Books: Mill Valley, CA, 1987.

(241) The Chemistry of the Metal-Carbon Bond; Hartley, F. R. Patai, S., Eds.; Wiley-Interscience: New York, 1985; Vol. 2

(242) Reactions of Coordinated Ligands; Braterman, P. S., Ed.; Plenum: New York, 1986; Vol 1.

(243) Bush, R. C.; Angelici, R. J. J. Am. Chem. Soc. 1986, 108, 2735

(244) Beck, W. Polyhedron, in press.

(245) Steil, P.; Nagel, U.; Beck, W. J. Organomet. Chem. 1988, 339 , 111 and literature cited therein.

(246) Snow, M. R.; Wimmer, F. L. Inorg. Chim. Acta 1980, 44, L189.

(247) Olgemöller, B.; Olgemöller, L.; Beck, W. Chem. Ber, 1981 114,2971

(248) Thewalt, U.; Klein, H.-P. J. Organomet. Chem. 1980, 194, 297
(249) Thewalt, U.; Lasser, W. J. Organomet. Chem. 1984, 276, 341. (250) Green, M. L. H.; Parkin, G.; Chen, M.; Prout, K. J. Chem. Soc., Dalton Trans. 1986, 2227.

(251) Boniface, S. M.; Clark, G. R.; Collins, T. J.; Roper, W. R. J. Organomet. Chem. 1981, 206, 109.

(252) Bauer, H.; Nagel, U.; Beck, W. J. Organomet. Chem. 1985 , $290,219$.

(253) Green, M.; Kirsch, H. P.; Stone, F. G. A.; Welch, A. J. J. Chem. Soc., Dalton Trans. 1977, 1755

(254) Lasser, W.; Thewalt, U. J. Organomet. Chem. 1986, 302, 201.

(255) Lasser, W.; Thewalt, U. J. Organomet. Chem. 1986, 311, 69.

(256) Legzdins, P.; Nurse, C. R. Inorg. Chem. 1982, 21, 3110.

(257) Curtis, M. D.; Butler, W. M. J. Chem. Soc., Chem. Commun. $1980,998$.

(258) Grandberg, K. I. Sov. J. Coord. Chem. (Engl. Transl.) 1984, $10,358$.

(259) Baldwin, J. E.; Haraldsson, G. G.; Jones, J. G. Inorg. Chim. Acta 1981, 51, 29.

(260) Kölle, U.; Fuss, B. Chem. Ber. 1984, 117, 753.

(261) Thewalt, U.; Döppert, K.; Lasser, W. J. Organomet. Chem. $1986,308,303$.

(262) (a) Hieber, W.; Leutert, F. Z. Anorg. Allg. Chem. 1932, 204, 145. (b) Kruck, Th.; Höfler, M.; Noack, M. Chem. Ber. 1966, $99,1153$.

(263) Beck, W.; Raab, K.; Nagel, U.; Steimann, M. Angew. Chem., Int. Ed. Engl. 1982, 21, 526 .

(264) Bennett, M. A.; Matheson, T. W.; Robertson, G. B.; Steffen W. L.; Turney, T. W. J. Chem. Soc., Chem. Commun. 1979, 32.

(265) Dewan, J. C.; Thompson, L. K. Can. J. Chem. 1982, 60, 121.

(266) Beck, W.; Sacher, W.; Nagel, U. Angew. Chem., Int. Ed. Engl. 1986, 25, 270 .

(267) Horn, E.; Snow, M. R. Aust. J. Chem. 1984, 37, 35

(268) Klein, H.-P.; Thewalt, U. J. Organomet. Chem. 1981, $206,69$.

(269) Hillhouse, G. L.; Haymore, B. L. Inorg. Chem. 1987, 26, 1876.

(270) Miguel, D.; Riera, V. J. Organomet. Chem. 1985, 293, 379.

271) Reger, D. L.; Swift, C. A. Organometallics 1984, 3, 876.

(272) Werner, H.; Weinand, R.; Otto, H. J. Organomet. Chem. 1986, $307,49$.

(273) Bird, C.; Booth, B. L.; Haszeldine, R. N. J. Chem. Soc. Dalton Trans. 1982, 517

(274) King, R. B.; Kapoor, R. N. J. Organomet. Chem. 1968, 15, 457.

(275) Grove, D. M.; Van Koten, G.; Ubbels, H. J. C.; Zoet, R.; Spek, A. L. J. Organomet. Chem. 1984, 263, C10.

(276) Aoyama, Y.; Yoshida, T.; Sakurai, K.; Ogoshi, H. J. Chem. Soc. Chem. Commun. 1983, 478

(277) Fischer, R. A.; Kneuper, H.-J.; Herrmann, W. A. J. Organomet. Chem. 1987, 330, 365

(278) Lee, K. Y.; Kuchynka, D. J.; Kochi, J. K. Organometallics $1987,6,1886$.

(279) (a) Shriver, D. F. Struct. Bonding (Berlin) 1966, 1, 32. (b) Kristoff, J. S.; Shriver, D. F. Inorg. Chem. 1973, 12, 1788. (c) Manzer, L. E.; Parshall, G. W. Ibid. 1976, 15, 3114

(280) Strope, D.; Shriver, D. F. Inorg. Chem. 1974, 13, 2652.

(281) Abys, J. A.; Ogar, G.; Risen, W. M., Jr. Inorg. Chem. 1981, 20 , 4446 .

(282) Fritz, P. M.; Beck, W., unpublished results.

(283) Fritz, P. M.; Steimann, M.; Beck, W. Chem. Ber. 1987, 120, 253.

(284) Tso C. T. Cutler A. R J Am. Chem Soc, 1986, 108,6069

(285) (a) Adams, H.; Bailey, N. A.; Gauntlett, J. T.; Winter, M. J. J. Chem. Soc., Chem. Commun. 1984, 1360." (b) Gauntlett, J. T.; Winter, M. J. Polyhedron 1986, 5,451

(286) (a) Bonnesen, P. V.; Baker, A. T.; Hersh, W. H. J. Am. Chem. Soc. 1986, 108, 8304. (b) Bonnesen, P. V.; Yau, P. K. L.; Hersh, W. H. Organometallics 1987, 6, 1587.

(287) Weinstock, I.; Floriani, C.; Chiesi-Villa, A.; Guastini, C. J. Am. Chem. Soc. 1986, 108, 8298.

(288) O'Connor, J. M.; Uhrhammer, R.; Rheingold, A. L. Organometallics 1987, 6,1987 . O'Connor, J. M.; Uhrhammer, R. J. Am. Chem. Soc. $1988,110,4448$.

(289) Götzfried, F.; Grenz, R.; Urban, G.; Beck, W. Chem. Ber. $1985,118,4179$.

(290) (a) Nesmeyanov, A. N.; Makarova, L. G.; Vinogradova, V. N.; Ustynyuk, N. A. Koord. Khim. 1977, 3, 62. (b) BurckettSt.Laurent, J. C. T. R.; Field, J. S.; Haines, R. J.; McMahon, M. J. Organomet. Chem. 1979, 181,117.

(291) Jones, P. G.; Sheldrick, G. M.; Usön, R.; Laguna, A. Acta Crystallogr, Sect. B 1980, B36, 1486.

(292) Mews, R. Angew. Chem., Int. Ed. Engl. 1977, 16, 56.

(293) Snow, M. R.; Wimmer, F. L. Aust. J. Chem. 1976, 29, 2349.

(294) Fischer, E. O.; Moser, E. Z. Anorg. Allg. Chem. 1966, 342,

(295) Tso, C. C.; Cutler, A. R. Organometallics 1985, 4, 1242.

(296) Davies, J. A.; Hartley, F. R.; Murray, S. G.; Pierce-Butler, M. A. J. Chem. Soc., Dalton Trans. 1983, 1305.

(297) Usön, R.; Forniēs, J.; Espinet, P.; Navarro, R. Inorg. Chim. Acta $1984,82,215$ 
(298) (a) Grove, D. M.; Van Koten, G.; Ubbels, H. J. C.; Spek, A. L. J. Am. Chem. Soc. 1982, 104, 4285. (b) Terheijden, J.; Van Koten, G.; Grove, D. M.; Vrieze, K.; Spek, A. L. J. Chem. Soc., Dalton Trans. 1987, 1359.

(299) Olgemöller, B.; Beck, W. Chem. Ber. 1981, 114, 2360

(300) Stolzenberg, H.; Fehlhammer, W. P. J. Organomet. Chem. 1982, 235, C7. Busetto, L.; Monari, M.; Palazzi, A.; Albano, V.; Demartin, F. J. Chem. Soc., Dalton Trans. 1983, 1849.

(301) Hecht, C.; Herdtweck, E.; Rohrmann, J.; Herrmann, W. A.; Beck, W.; Fritz, P. M. J. Organomet. Chem. 1987, 330, 389.

(302) Fritz, P. M.; Beck, W.; Nagel, U.; Polborn, K.; Herrmann, W. A.; Hecht, C.; Rohrmann, J. Z. Naturforsch., B: Anorg. Chem. Org. Chem. 1988, 43, 665.

(303) Weiss, A.; Nagorsen, G.; Weiss, A. Z. Anorg. Allg. Chem. 1953, 274, 151. Puff, H.; Küster, J. Naturwissenschaften. 1962, 49, 299. Kamenar, B.; Kaitner, B.; Pocev, St. J. Chem. Soc., Dalton Trans. 1985, 2457.

(304) Kowala, C.; Swan, J. M. Aust. J. Chem. 1966, 19, 547. Abel, E. W.; Jenkins, C. R. J. Organomet. Chem. 1968, 14, 285. Schmidbaur, H.; Franke, R.; Eberlein, J. Chem.-Ztg. 1975, 99, 91.

(305) (a) Jones, P. G.; Sheldrick, G. M.; Hädicke, E. Acta Crystallogr., Sect. B 1980, 36, 2777. (b) Lensch, C.; Jones, P. G.; Sheldrick, G. M. Z. Naturforsch., B 1982, 37b, 944 .

(306) Hartmann, G.; Mews, R.; Sheldrick, G. M. Angew. Chem., Int. Ed. Engl. 1983, 22, 723.

(307) Kuhn, N.; Schumann, H. J. Organomet. Chem. 1985, 287, 345.

(308) Appel, M.; Heidrich, J.; Beck, W. Chem. Ber. 1987, 120, 1087.

(309) Kolobova, N. E.; Skripkin, V. V.; Rozantseva, T. V.; Struchkov, Yu. T.; Aleksandrov, G. G.; Andrianov, V. G. J. Organomet. Chem. 1981, 218, 351 .

(310) Afzal, D.; Lenhert, P. G.; Lukehart, C. M. J. Am. Chem. Soc. 1984, 106, 3050. Afzal, D.; Lukehart, C. M. Organometallics $1987,6,546$.

(311) Davis, J. H., Jr.; Lukehart, C. M. Organometallics 1984, 3, 1763.

(312) Venanzi, L. M. Coord. Chem. Rev. 1982, 43, 251.

(313) Albinati, A.; Lehner, H.; Venanzi, L. M.; Wolfer, M. Inorg. Chem. 1987, 26, 3933 and references therein.

(314) Howarth, O. W.; McAteer, C. H.; Moore, P.; Morris, G. E.; Alcock, N. W. J. Chem. Soc., Dalton Trans. 1982, 541.

(315) Albinati, A.; Naegeli, R.; Togni, A.; Venanzi, L. M. J. Organomet. Chem. 1987, 330, 85.

(316) Markham, J.; Cutler, A. Organometallics 1984, 3, 736.

(317) Hames, B. W.; Legzdins, P. Organometallics 1982, 1, 116.

(318) Werner, H.; Wolf, J.; Höhn, A. J. Organomet. Chem. 1985, 287,395 .

(319) Aspinall, H. C.; Deeming, A. J. J. Chem. Soc., Dalton Trans. 1985,743 .

(320) (a) LaCroce, S. J.; Cutler, A. R. J. Am. Chem. Soc. 1982, 104, 2312. (b) Markham, J.; Tolman, W.; Menard, K.; Cutler, A. J. Organomet. Chem. 1985, 294, 45.

(321) Sünkel, K.; Schloter, K.; Beck, W.; Ackermann, K.; Schubert, U. J. Organomet. Chem. 1983, 241,333 .
(322) Butts, S. B.; Strauss, S. H.; Holt, E. M.; Stimson, R. E.; Alcock, N. W.; Shriver, D. F. J. Am. Chem. Soc. 1980, 102, 5093.

(323) Akita, M.; Kawahara, T.; Moro-oka, Y. J. Chem. Soc., Chem. Commun. 1987, 1356.

(324) Sacher, W.; Beck, W., unpublished results.

(325) Haines, R. J.; DuPreez, A. L. J. Organomet. Chem. 1975, 84, 357.

(326) Raab, K.; Appel, M.; Beck, W. J. Organomet. Chem. 1985, $291, \mathrm{C} 28$.

(327) Werner, H.; Otto, H. Chem. Ber. 1986, 119, 3866.

(328) Werner, H.; Weinand, R. Z. Naturforsch., B 1983, 38b, 1518.

(329) Ballivet-Tkatchenko, D.; Nickel, B.; Rassat, A.; VincentVaucquelin, J. Inorg. Chem. 1986, 25, 3497.

(330) Oehme, G. J. Prakt. Chem. 1984, 326, 779.

(331) (a) Seyferth, K.; Taube, R. J. Organomet. Chem. 1984, 268 , 155. (b) Seyferth, K.; Taube, R. J. Organomet. Chem. 1984 $262,179$.

(332) (a) Tanabe, Y.; Mukaiyama, T. Chem. Lett. 1984, 1867. (b) Tanabe, Y.; Mukaiyama, T. Chem. Lett. 1985, 673.

(333) Honeychuck, R. V.; Bonnesen, P. V.; Favahi, J.; Hersh, W. H. J. Org. Chem. 1987, 52, 5293

(334) Siedle, A. R.; Markell, C. G.; Lyon, P. A.; Hodgson, K. O.; Roe, A. L. Inorg. Chem. 1987, 26, 219.

(335) Yang, K. J. Chin C. S. Inorg. Chem. 1987, $26,2732$.

(336) (a) Howarth, O. W.; McAteer, C. H.; Moore, P.; Morris, G. E J. Chem. Soc., Dalton Trans. 1984, 1171. (b) Crabtree, R. H.; Dion, R. P.; Gibboni, D. J.; McGrath, D. V.; Holt, E. M. $J$ Am. Chem. Soc. 1986, 108, 7222. (c) Halpern, J. In Asymmetric Synthesis; Morrison, J. D., Ed.; Academic: Orlando, 1985; Vol. 5. (d) Crabtree, R. H. Acc. Chem. Res. 1979, 12 331.

(337) Klapötke, Th. Polyhedron 1988, 7, 1221.

(338) Klapötke, Th.; Thewalt, U. J. Organomet. Chem., in press.

(339) Klapötke, Th. Polyhedron, in press.

(340) Klapötke, Th. Inorg. Chim. Acta, in press.

(341) Cutler, A. R.; Todaro, A. B. Organometallics 1988, 7, 1782

(342) Schweiger, M.; Beck, W., unpublished results.

(343) Fernândez, J. M.; Gladysz, J. A. Organometallics, in press.

(344) Winter, C. H.; Arif, A. M.; Gladysz, J. A. Organometallics, in press.

(345) Buhro, W. E.; Zwick, B. D.; Georgiou, S.; Hutchinson, J. P.; Gladysz, J. A. J. Am. Chem. Soc. 1988, 110, 2427.

(346) Bodner, G. S.; Fernândez, J. M.; Arif, A. M.; Gladysz, J. A J. Am. Chem. Soc. 1988, 110, 4082 .

(347) Honeychuck, R. V.; Hersh, W. H., submitted to J. Am. Chem. Soc.

(348) Bond, A. M.; Ellis, S. R.; Hollenkamp, A. F. J. Am. Chem. Soc. $1988,110,5293$.

(349) Amarasekera, J.; Rauchfuss, T. B.; Wilson, S. R. J. Am. Chem. Soc. 1988, 110, 2332

(350) Antberg, M.; Dahlenburg, L.; Frosin, K.-M.; Höck, N. Chem. Ber. 1988, 121, 859.

(351) Pearson, A. J.; Richards, I. C. J. Organomet. Chem. 1983, 258, C41.

(352) Weidmann, T.; Beck, W., unpublished results. 